\title{
Control Strategies for a Multi-strain Epidemic Model
}

\author{
Yuan Lou' ${ }^{1,2} \cdot$ Rachidi B. Salako 3
}

Received: 21 June 2021 / Accepted: 12 October 2021 / Published online: 27 November 2021

(c) The Author(s), under exclusive licence to Society for Mathematical Biology 2021

\begin{abstract}
This article studies a multi-strain epidemic model with diffusion and environmental heterogeneity. We address the question of a control strategy for multiple strains of the infectious disease by investigating how the local distributions of the transmission and recovery rates affect the dynamics of the disease. Our study covers both full model (in which case the diffusion rates for all subgroups of the population are positive) and the ODE-PDE case (in which case we require a total lock-down of the susceptible subgroup and allow the infected subgroups to have positive diffusion rates). In each case, a basic reproduction number of the epidemic model is defined and it is shown that if this reproduction number is less than one then the disease will be eradicated in the long run. On the other hand, if the reproduction number is greater than one, then the disease will become permanent. Moreover, we show that when the disease is permanent, creating a common safety area against all strains and lowering the diffusion rate of the susceptible subgroup will result in reducing the number of infected populations. Numerical simulations are presented to support our theoretical findings.
\end{abstract}

Keywords Infectious disease - Reaction-diffusion - Asymptotic behavior · Competition-exclusion

Mathematics Subject Classification 92D25 $\cdot 35 \mathrm{~B} 40 \cdot 35 \mathrm{~K} 57$

Rachidi B. Salako

rachidi.salako@unlv.edu

Yuan Lou

lou@math.ohio-state.edu

1 School of Mathematical Sciences, CMA-Shanghai, Shanghai Jiao Tong University, Shanghai 200240, People's Republic of China

2 Department of Mathematics, Ohio State University, Columbus, OH 43210, USA

3 Department of Mathematical Sciences, University of Nevada, Las Vegas, Las Vegas, NV 89154, USA 


\section{Introduction}

Implementing an effective strategy to limit infection, or if possible, completely eradicating an infectious disease has always been a challenging question for public health. In some urgent situations, portions of the population are completely locked down in order to control an outbreak as was the case during the early of Covid-19 in some countries around the world. But the safety measures of restricting population movement often result in an increased financial burden on the society. In addition, these safety measures substantially affect people's daily activities and capacity for in-person social interactions. In some cases, people might experience mental health issues and anxiety as consequences of the imposed control strategies. Although some of the aforementioned negative impacts can be linked to the implementation of the safety measures requiring the restriction of population movement, they are still widely used by the government in the fight against the spreading of deadly infectious diseases. Our goal of this paper is to use mathematical models to assess the effectiveness of the lock-down strategies against multiple strains.

In this work, we investigate the dynamics of the multi-strain PDE-SIS epidemic system

$$
\begin{cases}\partial_{t} S=d_{\mathrm{S}} \Delta S+\sum_{i=1}^{k} \gamma_{i}(x) I_{i}-\frac{S \sum_{i=1}^{k} \beta_{i}(x) I_{i}}{S+\sum_{j=1}^{k} I_{j}} & x \in \Omega, t>0, \\ \partial_{t} I_{i}=d_{i} \Delta I_{i}-\gamma_{i}(x) I_{i}+\beta_{i}(x) \frac{S I_{i}}{S+\sum_{j=1}^{k} I_{j}} & x \in \Omega, t>0, \\ d_{\mathrm{S}} \frac{\partial S}{\partial \vec{n}}=d_{i} \frac{\partial I_{i}}{\partial \vec{n}}=0 & x \in \partial \Omega, t>0, \\ N=\int_{\Omega}\left[S(0, x)+\sum_{i=1}^{k} I_{i}(0, x)\right] \mathrm{d} x>0 & \end{cases}
$$

in a bounded domain $\Omega \subset \mathbb{R}^{n}$ with smooth boundary $\partial \Omega, n$ is a positive integer, and $\vec{n}$ is the outer unit normal vector on $\partial \Omega$. The PDE-SIS system (1) supposes that there are $k \geq 1$ strains of the disease with $I_{i}(t, x)$ denoting the density of the infected population with the $i$ th strain for each $i=1, \ldots, k$, and $S(t, x)$ is the density function of the susceptible subgroup of the population. For each $i=1, \ldots, k, \beta_{i}$ and $\gamma_{i}$ are positive Hölder continuous functions on $\bar{\Omega}$ and denote the transmission and recovery rates of the $i$ th strain of the disease, respectively. $N$, a given positive number, denotes the total size of the population. The constant $d_{\mathrm{S}}>0$ is the diffusion rate of the susceptible group, while the positive constant $d_{i}, i=1, \ldots, k$, is the diffusion rate of the infected population with the $i$ th strain. It is assumed that there is no co-infection of the host with two or more strains of the disease. We further impose no-flux boundary condition on $\partial \Omega$.

The PDE-SIS model (1) is a generalization of the multi-strain ODE-SIS infection disease system studied in Bremermann and Thieme (1989). The single strain model of (1), that is $k=1$, was first proposed by Allen et al. (2008) to study the impact of spatial heterogeneity of the environment and movement of individuals on the persistence and extinction of a disease. In Allen et al. (2008), the basic reproduction number $\mathcal{R}_{0}$ is defined for the single strain PDE-SIS model and it is established there that besides the disease-free equilibrium, the system has a (unique) endemic equilibrium if and only if $\mathcal{R}_{0}>1$. The basic reproduction number $\mathcal{R}_{0}$ of the single strain model is 
independent of $d_{\mathrm{S}}$, the diffusion rate of the susceptible subgroup, and depends solely on the diffusion rate of the infected group, the transmission and recovery rates. Allen et al. further introduced the concept of low risk region in Allen et al. (2008), i.e., locations where the local basic reproduction number is smaller than one. They showed that if there is a low risk region, restricting the movement of the susceptible population could substantially reduce the size of the infected populations. The stability of the endemic equilibrium of the single strain model is studied in Peng and Liu (2009). We refer to Cui et al. (2017), Cui and Lou (2016), Deng (2019), Deng and Wu (2016), Gao (2019), Ge et al. (2015), Li et al. (2017), Li et al. (2020), Liu and Lou (2021), Peng (2009), Peng and Yi (2013), Peng and Zhao (2012), Song et al. (2018), Wu and Zou (2016) and references therein for more recent progress.

The papers Tuncer and Martcheva (2012), Wu et al. (2017) considered the twostrain model, that is $k=2$. In Tuncer and Martcheva (2012), the authors introduced the invasion numbers of the two-strain PDE-SIS model (1) and showed analytically and numerically that if both invasion numbers are larger than one, then there is a coexistence equilibrium. The authors of Wu et al. (2017) also studied the two-strain system and investigated analytically under what conditions the model leads to competitive exclusion, and what characteristics of the model imply coexistence. In particular, it is shown in Wu et al. (2017) that if the transmission and recovery rates are constants, the two-strain PDE-SIS model (1) does not have a coexistence endemic equilibrium. It is also shown in Wu et al. (2017) that if all the diffusion rates are equal and sufficiently small, then both strains of the disease may coexist provided that each strain has a nonempty region in which its local reproduction number is the largest and it is also greater than one. We refer the interested reader to Ackleh and Allen (2003), Ackleh and Allen (2005), Ackleh et al. (2016), Levin and Pimentel (1981), MenaLorca and Velasco-Hernandez (1995) and the references therein for some studies of the multi-strain ODE infectious disease models.

The main aim of this paper is to study how to implement control strategies when there are multiple strains. For a single strain, the earlier works of Allen et al. (2008) suggested that decreasing the diffusion rate of the susceptible population could be an effective control strategy, provided that there is a low risk region. A natural question is whether such control strategy remains effective for multiple strains. Our findings suggest that the existence of a common low risk region for all strains is critical. More specifically,

1. Even if the infectious disease becomes endemic, as long as there is a common low risk area for all its strains, the total size of the infected individuals can be significantly reduced by restricting the movement of the susceptible subgroup of the population (see Theorem 2.3).

2. In the absence of a common low risk area for all strains of the disease, it is possible that each strain persists no matter how the movement of the susceptible subgroup is being restricted (see Theorem 2.4).

As noted above, these results suggest that creating a common low-risk region and restricting the movement of the susceptible subgroup can be effective strategies for the control of multi-strain infectious diseases. Furthermore, our result (Theorem 2.5) indicates that a complete lock-down of the susceptible hosts only, that is $d_{\mathrm{S}}=0$ 
and $d_{i}>0$ for each $i=1, \ldots, k$, will substantially weaken the capability of the disease to spread if there is a common low risk region. We hope that these studies can provide some insight into the impacts of movement of populations and environmental heterogeneity upon the spatial spread of multi-strain infectious diseases.

The rest of the paper is organized as follows. Section 2 is devoted to the statement of the main theoretical results. Section 3 provides biological interpretations and numerical simulations of our theoretical results. The proofs of the main results are presented in Appendices A, B and C.

\section{Main Results}

In this section, we state our results. We first introduce some notations and definitions. Next, we state our results when $d_{\mathrm{S}}>0$ and then discuss the results for the case $d_{\mathrm{S}}=0$.

\subsection{Notations and Definitions}

In the current subsection, we introduce some notations and definitions. Let $C(\bar{\Omega})$ denote the Banach space of uniformly continuous functions on $\Omega$ endowed with the sup-norm. We denote by $C^{+}(\bar{\Omega})$ the closed subset of $C(\bar{\Omega})$ consisting of nonnegative functions. Given $\left(S(0, \cdot), I_{1}(0, \cdot), \ldots, I_{k}(0, \cdot)\right) \in\left[C^{+}(\bar{\Omega})\right]^{k+1}$, we denote by $\left(S(t, \cdot), I_{1}(t, \cdot), \ldots, I_{k}(t, \cdot)\right)$ the unique classical solution of (1) with initial condition $\left(S(0, \cdot), I_{1}(0, \cdot), \ldots, I_{k}(0, \cdot)\right)$ and defined on its maximal interval of existence $\left[0, T_{\max }\right)$, where $T_{\max } \in(0, \infty]$. It follows from the maximum principle that $\left(S(t, \cdot), I_{1}(t, \cdot), \ldots, I_{k}(t, \cdot)\right) \in\left[C^{+}(\bar{\Omega})\right]^{k+1}$ for every $t \in\left(0, T_{\max }\right)$ and that $T_{\max }=\infty$. Throughout this work, we are only interested in non-negative solutions of (1). For convenience, we introduce the hypothesis

$$
\begin{aligned}
& \text { (A) }\left(S(0, \cdot), I_{1}(0, \cdot), \ldots, I_{k}(0, \cdot)\right) \in\left[C^{+}(\bar{\Omega})\right]^{k+1} \\
& \text { and } N=\int_{\Omega}\left[S(0, x)+\sum_{i=1}^{k} I_{i}(0, x)\right] \mathrm{d} x .
\end{aligned}
$$

It is important to note that hypothesis (A) is invariant for solutions of (1). Throughout this work, we shall always suppose that hypothesis (A) holds. We say that a classical solution $\left(S(t, x), I_{1}(t, x), \ldots, I_{k}(t, x)\right)$ of (1) has a positive initial condition if $I_{i}(0, \cdot) \geq, \neq 0$ for each $i=1, \ldots, k$ and $S(0, \cdot) \geq, \neq 0$.

A function $\left(S(x), I_{1}(x), \ldots, I_{k}(x)\right)$ is said to be an equilibrium solution of (1) if it is a time-independent solution of (1), that is, it solves the system

$$
\begin{cases}0=d_{\mathrm{S}} \Delta S+\sum_{i=1}^{k} \gamma_{i} I_{i}-\frac{S \sum_{i=1}^{k} \beta_{i} I_{i}}{S+\sum_{j=1}^{k} I_{j}} & x \in \Omega, \\ 0=d_{i} \Delta I_{i}+\beta_{i} \frac{S I_{i}}{S+\sum_{j=1}^{k} I_{j}}-\gamma_{i} I_{i} & x \in \Omega, i=1, \ldots, k, \\ 0=d_{\mathrm{S}} \frac{\partial S}{\partial \vec{n}}=d_{i} \frac{\partial I_{i}}{\partial \vec{n}} & x \in \partial \Omega, i=1, \ldots, k, \\ N=\int_{\Omega}\left[S+\sum_{i=1}^{k} I_{i}\right] . & \end{cases}
$$


A solution of system (2) of the form $(S, 0, \ldots, 0)$ is called a disease-free equilibrium (DFE). It is easy to see that the only DFE of (1) is $\left(\frac{N}{|\Omega|}, 0, \ldots, 0\right)$ when $d_{\mathrm{S}}>0$. An equilibrium solution for which at least one of its $I_{i}$ component being positive is called an endemic equilibrium (EE). A coexistence EE is an EE for which $I_{i}>0$ for every $i=1, \ldots, k$.

For each $i=1, \ldots, k$, set

$$
\mathcal{R}_{0, i}:=\sup \left\{\frac{\int_{\Omega} \beta_{i} \varphi^{2}}{\int_{\Omega}\left[d_{i}|\nabla \varphi|^{2}+\gamma_{i} \varphi^{2}\right]}: \varphi \in H^{1}(\Omega) \backslash\{0\}\right\} .
$$

Thanks to the work of Allen et al. (2008) on the single-strain PDE-SIS model (1) with $d_{\mathrm{S}}>0, \mathcal{R}_{0, i}$ is the basic reproduction number of the $i$ th strain when considered as the only strain of the disease. It is shown in Allen et al. (2008) that for every $i=$ $1, \ldots, k$, a single-strain EE, $\left(S^{*}, 0, \ldots, I_{i}^{*}, 0, \ldots, 0\right)$, exists if and only if $\mathcal{R}_{0, i}>1$. For convenience, set

$$
\mathcal{R}_{0}:=\max \left\{\mathcal{R}_{0, i}: i=1, \ldots, k\right\}
$$

As we shall see later, $\mathcal{R}_{0}$ is the basic reproduction number of the multi-strain PDE-SIS model (1) when $d_{\mathrm{S}}>0$.

Next, for each $i=1, \ldots, k$, consider the "local basic reproduction number" function for the $i$ th strain

$$
\Re_{i}(x):=\frac{\beta_{i}(x)}{\gamma_{i}(x)} \quad \forall x \in \bar{\Omega}
$$

and define the sets

$$
H_{i}^{-}:=\left\{x \in \bar{\Omega}: \Re_{i}(x)<1\right\} \text { and } H_{i}^{+}:=\left\{x \in \bar{\Omega}: \mathfrak{R}_{i}(x)>1\right\}
$$

For $i$ th strain, we refer $H_{i}^{-}$as the low-risk area and $H_{i}^{+}$as its high-risk area, respectively.

As in the above, we also introduce the function

$$
\mathfrak{R}(x):=\max \left\{\Re_{i}(x): i=1, \ldots, k\right\} \quad x \in \bar{\Omega},
$$

the set

$$
H^{-}:=\{x \in \bar{\Omega}: \mathfrak{R}(x)<1\}=\cap_{i=1}^{k} H_{i}^{-},
$$

and the quantity

$$
\mathfrak{R}_{0}:=\min _{x \in \bar{\Omega}} \mathfrak{R}(x)
$$

The function $\Re(x)$ can be understood as the local reproduction number of the disease while the set $H^{-}$is the common safety area for all strains. This set turns out to be of 
particular importance when we study the multi-strain model (1) with $d_{\mathrm{S}}=0$. Observe that $H^{-}$is nonempty if and only if $\Re_{0}<1$. In studying the PDE-SIS model with $d_{\mathrm{S}}=0$, the number $\mathfrak{R}_{0}$ will be of interest.

Finally, to capture the region of the dominance of $i$ th strain, we introduce the sets

$$
\Sigma_{i}:=\left\{x \in \bar{\Omega}: \mathfrak{R}_{i}(x)>\max \left\{\mathfrak{R}_{j}(x): j \neq i, j=1, \ldots, k\right\}\right\}, i=1, \ldots, k
$$

Clearly, for each $i=1, \ldots, k$, the $i$ th strain has the highest local basic reproduction number on the set $\Sigma_{i}$. Whence $\Sigma_{i}$ will be referred to as the dominant area for $i$ th strain.

\subsubsection{Main Results When $d_{\mathrm{S}}>0$}

All the results stated in the current subsection are for the case $d_{\mathrm{S}}>0$ and $d_{i}>0$ for each $i=1, \ldots, k$. Our first result is on the stability of the DFE, which depends on the basic reproduction number $\mathcal{R}_{0}$.

Theorem 2.1 Let $k \geq 2$ and $\left(S(t, \cdot), I_{1}(t, \cdot), \ldots, I_{k}(t, \cdot)\right)$ be a classical solution of (1) with a positive initial data satisfying hypothesis (A). The following conclusions hold:

(i) If $\mathcal{R}_{0, i} \leq 1$ for some $i \in\{1, \ldots, k\}$, then $\left\|I_{i}(t, \cdot)\right\|_{\infty} \rightarrow 0$ as $t \rightarrow \infty$.

(ii) If $\mathcal{R}_{0} \leq 1$, then $\left\|S(t, \cdot)-\frac{N}{|\Omega|}\right\|_{\infty}+\sum_{i=1}^{k}\left\|I_{i}(t, \cdot)\right\|_{\infty} \rightarrow 0$ as $t \rightarrow \infty$.

(iii) If $\mathcal{R}_{0}>1$, then there is some constant $m_{*}>0$, independent of initial data, such that

$$
\liminf _{t \rightarrow \infty} \min _{x \in \bar{\Omega}} \sum_{i=1}^{k} I_{i}(t, x) \geq m_{*} .
$$

It follows from Theorem 2.1-(iii) that the disease persists when $\mathcal{R}_{0}>1$ in the sense of (3). The next result provides sufficient conditions for the extinction of some strains of the disease even if their reproduction numbers are greater than one.

Theorem 2.2 Let $k \geq 2, d_{1}=\cdots=d_{k}=d$ and suppose that $\mathcal{R}_{0}>1$, so that the disease persists. Let $\left(S(t, \cdot), I_{1}(t, \cdot), \ldots, I_{k}(t, \cdot)\right)$ be a classical solution of $(1)$ with a positive initial data satisfying hypothesis (A). The following conclusions hold:

(i) If $\beta_{1}=\cdots=\beta_{k}$ and $\gamma_{1}<\min _{2 \leq i \leq k} \gamma_{i}$, then $\left\|\left(I_{2}(t, \cdot), \ldots, I_{k}(t, \cdot)\right)\right\|_{\infty} \rightarrow 0$ as $t \rightarrow \infty$.

(ii) If $\gamma_{1}=\cdots=\gamma_{k}$ and $\beta_{1}>\max _{2 \leq i \leq k} \beta_{i}$, then $\left\|\left(I_{2}(t, \cdot), \ldots, I_{k}(t, \cdot)\right)\right\|_{\infty} \rightarrow 0$ as $t \rightarrow \infty$.

(iii) If $\beta_{i}=\hat{\beta}_{i} g, \gamma_{i}=\hat{\gamma}_{i} h$, where $\hat{\beta}_{i}$ and $\hat{\gamma}_{i}$ are positive constants for each $i=1, \ldots, k$ and $g, h$ are positive Hölder continuous functions, $\hat{\beta}_{1} \geq$ $\max \left\{\hat{\beta}_{j}: j=2, \ldots, k\right\}$, and $\frac{\hat{\beta}_{1}}{\hat{\gamma}_{1}}>\max \left\{\frac{\hat{\beta}_{j}}{\hat{\gamma}_{j}}: j=2, \ldots, k\right\}$, then $\left\|\left(I_{2}(t, \cdot), \ldots, I_{k}(t, \cdot)\right)\right\|_{\infty} \rightarrow 0$ as $t \rightarrow \infty$. 
(iv) If $d_{\mathrm{S}}=d, \mathfrak{R}_{i}$ is constant for all $i=1, \ldots, k$, with $\mathfrak{R}_{1}>\max \left\{\mathfrak{R}_{j}: j=\right.$ $2, \ldots, k\}$, then $\left(S(t, \cdot), I_{1}(t, \cdot), \ldots, I_{k}(t, \cdot)\right) \rightarrow\left(\frac{N}{|\Omega| \Re_{1}}, \frac{N}{|\Omega|}\left(1-\frac{1}{\Re_{1}}\right), 0, \ldots, 0\right)$ as $t \rightarrow \infty$, uniformly in $x \in \Omega$.

We point out that if $d_{\mathrm{S}}=d$, in addition to the hypotheses of Theorem 2.2 ( $\mathrm{i}$ ), ( $\mathrm{ii}$ ), and ( ii i), it can be shown that solutions will converge to the first single-strain EE as $t \rightarrow \infty$. It would be interesting to know whether the results of Theorem 2.2 hold for strains with different diffusion rates. In this direction, we would like to point out that in (Tuncer and Martcheva 2012, Theorem 3.2), it is established that the two-strain PDE-SIS model has no coexistence EE for any choice of diffusion rates $d_{1}, d_{2}$ and $d_{\mathrm{S}}$ when the infection and recovery rates are all positive constant numbers. Also, it can be shown (see Bremermann and Thieme 1989) that if $\left(S(t), I_{1}(t), \ldots, I_{k}(t)\right)$ is a positive solution of the ODE-system of (1), that is no diffusion, the population is uniformly distributed over its habitat and the infection and recovery rates are positive constant numbers, then

$$
I_{i}(t)=I_{i}(0)\left[\frac{I_{j}(t)}{I_{j}(0)}\right]^{\frac{\beta_{i}}{\beta_{j}}} e^{\left(\frac{1}{\Re_{j}}-\frac{1}{\Re_{i}}\right) \beta_{i} t} \forall t>0, i, j=1, \ldots, k
$$

It follows from Eq. (4) that if $\Re_{j}>\Re_{i}$, then $I_{i}(t) \rightarrow 0$ as $t \rightarrow \infty$. This shows

that for the multi-strain ODE-SIS model, the strain with the largest reproduction number outcompetes the other strains and drive them to extinction. This is known as the competition exclusion principle. Hence, Theorem 2.2 confirms the competition exclusion principle when the local basic reproduction functions $\mathfrak{R}_{i}$ are constant positive numbers and the susceptible host and the infected population all have equal diffusion rates. It is worth mentioning that Wu et al. (2017) also shows the competition exclusion principle for the two-strain PDE-SIS model (1) when both the recovery and infection rates are positive constant numbers, the diffusion rates are equal, $\mathfrak{R}_{1}>\mathfrak{R}_{2}>1$ and $1+\frac{1}{\mathfrak{R}_{1}}<\frac{2}{\mathfrak{R}_{2}}$.

It is important to note that $\mathcal{R}_{0}$ is independent of the susceptible host diffusion rate $d_{\mathrm{S}}$, which implies that the persistence of the disease and hence the existence of single strain EE is not affected by the susceptible host movement. So, it becomes interesting to know whether a total lock-down of the susceptible host or a limitation of its diffusion rate are good control strategies for reducing the impact of the disease within the population. To this end, we study the asymptotic limit of EE when $d_{\mathrm{S}}$ tends to 0 . The case of $d_{\mathrm{S}}=0$ will be discussed in the next subsection when we present our results for that case. An interesting result by Allen et al. (2008), Cui et al. (2017) for the single-strain model states that in environment with nonempty low-risk area, the size of infected population at equilibrium is proportional to the susceptible host movement rate $d_{\mathrm{S}}$ as $d_{\mathrm{S}}$ tends to zero. Our next result shows that a similar result holds for the multi-strain model (1).

Theorem 2.3 Assume that $H^{-} \neq \emptyset$. Then there exists a positive constant $M$, independent of $d_{\mathrm{S}}$, such that if $\left(S(x), I_{1}(x), \ldots, I_{k}(x)\right)$ is a coexistence EE of $(1)$, it holds 
that

$$
\frac{1}{M} \leq \frac{\sum_{i=1}^{k} I_{i}(x)}{d_{\mathrm{S}}} \leq M \quad \forall 0<d_{\mathrm{S}}<1, x \in \Omega .
$$

Theorem 2.3 shows that in an environment with a nonempty common safety area for all the strains of the disease, restricting the susceptible group movement helps to reduce the impact of the disease on the population, at least at the steady-state level. It turns out that when $H^{-}=\emptyset$, the assertion of Theorem 2.3 does not hold in general. In this direction, we have the following result:

Theorem 2.4 Let $k=2$ and suppose that $\mathfrak{R}>1$ on $\bar{\Omega}, \mathfrak{R}_{1}-\mathfrak{R}_{2}$ changes sign on $\Omega, \frac{\beta_{1}}{\beta_{2}}$ is constant, and $\beta_{i}, \gamma_{i} \in C^{2}(\bar{\Omega})$ for $i=1,2$. Let $D_{1}$ and $D_{2}$ be two given positive numbers and set $d_{i}=\varepsilon D_{i}, i=1,2$. There is $\tilde{\varepsilon}^{*}>0$, such that for every $0<\varepsilon<\tilde{\varepsilon}^{*}$, there is $d_{\varepsilon}^{*}>0$ such that (1) has a coexistence EE, denoted as $\left(S^{*}\left(\cdot, d_{\mathrm{S}}, \varepsilon\right), I_{1}^{*}\left(\cdot, d_{\mathrm{S}}, \varepsilon\right), I_{2}^{*}\left(\cdot, d_{\mathrm{S}}, \varepsilon\right)\right)$, for every $0<d_{\mathrm{S}}<d_{\varepsilon}^{*}$. Furthermore, for every $0<\varepsilon<\tilde{\varepsilon}^{*},\left(S^{*}\left(\cdot, d_{\mathrm{S}}, \varepsilon\right), I_{1}^{*}\left(\cdot, d_{\mathrm{S}}, \varepsilon\right), I_{2}^{*}\left(\cdot, d_{\mathrm{S}}, \varepsilon\right)\right) \rightarrow\left(S^{*}(\cdot, \varepsilon), I_{1}^{*}(\cdot, \varepsilon), I_{2}^{*}(\cdot, \varepsilon)\right)$ as $d_{\mathrm{S}} \rightarrow 0$ uniformly in $\Omega$, where $\left(S^{*}(\cdot, \varepsilon), I_{1}^{*}(\cdot, \varepsilon), I_{2}^{*}(\cdot, \varepsilon)\right)$ is the unique positive solution of

$$
\left\{\begin{array}{l}
S^{*}(\cdot, \varepsilon)=\frac{\sum_{i=1}^{2} \gamma_{i} I_{i}^{*}(\cdot, \varepsilon) \cdot \sum_{i=1}^{2} I_{i}^{*}(\cdot, \varepsilon)}{\sum_{i=1}^{2}\left(\Re_{i}-1\right) \gamma_{i} I_{i}^{*}(\cdot, \varepsilon)} \quad x \in \Omega, \\
0=d_{i} \Delta I_{i}^{*}+\left(\beta_{i} \frac{\sum_{i=1}^{2} \gamma_{i} I_{i}^{*}}{\sum_{i=1}^{2} \beta_{i} I_{i}^{*}}-\gamma_{i}\right) I_{i}^{*} \quad x \in \Omega, i=1,2, \\
0=\partial_{\vec{n}} I_{i}^{*} \quad x \in \partial \Omega, i=1,2, \\
0<\min \left\{S^{*}(x, \varepsilon), I_{1}^{*}(x, \varepsilon), I_{2}^{*}(x, \varepsilon)\right\} \quad x \in \bar{\Omega} .
\end{array}\right.
$$

For $k=2, \Re_{1}-\mathfrak{R}_{2}$ changes sign if and only if both $\Sigma_{1}$ and $\Sigma_{2}$ are nonempty. Recall that $\Sigma_{i}$ is the dominant area of the $i$ th strain. Hence, Theorem 2.4 illustrates that if each strain has a nonempty dominant area but there is no common safety zone for both strains, it is possible for two strains to be permanent even if the diffusion rate of the susceptible host is significantly small. In fact, the assertion of Theorem 2.4 follows from Theorem 4.5 (see Sect. 4.2) which provides sufficient hypotheses for the existence of coexistence EE when the rate of movement for the susceptible population is small.

\subsubsection{Main Results When $d_{s}=0$}

All the results stated in the current subsection are for the case $d_{\mathrm{S}}=0$ and $d_{i}>0$ for each $i=1, \ldots, k$. Our first result is on the extinction of the disease when $\mathfrak{R}_{0}<1$.

Theorem 2.5 Suppose that $\mathfrak{R}_{0}<1$. Let $\left(S(t, x), I_{1}(t, x), \ldots, I_{k}(t, x)\right)$ be a positive classical solution of (1). Then there is a non-negative function $S^{*} \in C(\bar{\Omega})$ such that

$$
\lim _{t \rightarrow \infty}\left[\left\|S(t, \cdot)-S^{*}(\cdot)\right\|_{\infty}+\sum_{i=1}^{k}\left\|I_{i}(t, \cdot)\right\|_{\infty}\right]=0 .
$$


Moreover, by introducing the set $J^{+}:=\left\{x \in \Omega: S^{*}(x)=0\right\}$, the followings hold:

(i) If $\mathcal{R}_{0}>1$, then the set $J^{+}$is nonempty and contained in $\cup_{i=1}^{k}\left(\Omega \cap H_{i}^{+}\right)$. Furthermore, both sets $J^{+}$and $\cup_{i=1}^{k}\left(\Omega \cap H_{i}^{+}\right) \backslash J^{+}$have positive measures.

(ii) If $\mathcal{R}_{0}<1$, then $\left\|\sum_{i=1}^{k} I(t, \cdot)\right\|_{\infty}$ decays exponentially as $t \rightarrow \infty$ and there is $m_{0}>0$ such that the set $J^{+}$is empty whenever $\left\|\sum_{i=1}^{k} I_{i}(0, \cdot)\right\|_{\infty}<m_{0} S(0, \cdot)$.

Assume that $\mathfrak{R}_{i}(x)=\mathfrak{R}(x)$ is independent of $i$ and that $d_{\mathrm{S}}=0$. By Theorem 2.5, if $\Re_{0}<1$, that is $H^{-}$is not empty, then (7) holds for every solution $\left(S(t, x), I_{1}(t, x), \ldots, I_{k}(t, x)\right)$ of (1). However, if $\Re_{0}>1$, that is $\bar{\Omega}=H_{1}^{+}$, system (1) has a continuum of coexistence endemic equilibria $\left(S_{e}(x), I_{e, 1}, \ldots, I_{e, k}\right)$ given by

$$
\begin{aligned}
& S_{e}(x)=\frac{N}{(\mathfrak{R}(x)-1)\left[\int_{\Omega} \frac{\mathfrak{R}}{\mathfrak{R}-1} d y\right]} \text { and } \\
& I_{e, i}(x) \equiv c_{i}>0 \text { with } \sum_{i=1}^{k} c_{i}=\frac{N}{\int_{\Omega} \frac{\mathfrak{R}}{\mathfrak{R}-1} d y} .
\end{aligned}
$$

Recall that the set $\mathrm{H}^{-}$is the common safety region for all the strains of the disease and that $\mathfrak{R}_{0}<1$ if and only if $H^{-}$is nonempty. Hence, Theorem 2.5 shows that if $d_{\mathrm{S}}=0$ and there is a nonempty common safety region for all the strains of the disease, then all the strains of the disease will be eradicated in the long run. This result seems to be new even for the case $k=1$. Our results below will show that the statement of Theorem 2.5 might not hold if $H^{-}=\emptyset$. The next two results are concerned with the single-strain model. The first result is on the existence of steady states for single strain, while the second result complements Theorem 2.5 and establishes the stability of the EE.

Theorem 2.6 Suppose that $k=1$.

(i) If $\Re_{0}<1$, then (1) has only disease-free equilibrium solutions which are of the form $\left(S^{*}(x), 0\right)$ where $S^{*} \in C(\bar{\Omega})$ and satisfies

$$
N=\int_{\Omega} S^{*} d x \text { and } S^{*}(x) \geq 0 \forall x \in \Omega .
$$

(ii) If $\mathfrak{R}_{0}>1$, then in addition to the disease-free equilibria listed in (i), (1) has a unique endemic equilibrium $\left(S_{e}(x), I_{e}(x)\right)$ given by

$$
S_{e}(x)=\frac{I_{e}}{\Re_{1}(x)-1} \quad \text { and } \quad I_{e}=\frac{N}{\int_{\Omega} \frac{\Re_{1}}{\Re_{1}-1} d x} .
$$

Remark 2.7 Suppose that $k=1$. We make the following comments about Theorem 2.6.

(i) If $\Re_{0}=1$ and $\beta_{1} /\left(\beta_{1}-\gamma_{1}\right) \notin L^{1}(\Omega)$, then (1) has only disease-free equilibrium solutions which are of the form $\left(S^{*}(\cdot), 0\right)$ with $S^{*}$ satisfying $(8)$. 
(ii) If $\Re_{0}=1$ and $\beta_{1} /\left(\beta_{1}-\gamma_{1}\right) \in L^{1}(\Omega)$, then (1) has measurable and unbounded $\mathrm{EE}$

$$
(S(x), I(x))=\left(\frac{N \chi_{H^{-}}(x)}{(\Re(x)-1) \int_{\Omega} \frac{\mathfrak{R}}{\mathfrak{R}-1} d y}, \frac{N}{\int_{\Omega} \frac{\mathfrak{R}}{\mathfrak{R}-1} d y}\right) \quad x \in \Omega
$$

where $\chi_{H^{-}}$denotes the indicator function of the set $H^{-}$. It is important to note that, when $\mathfrak{R}_{0} \leq 1$, continuous disease-free equilibrium are the only continuous equilibrium solutions of (1).

We complete the list of results on the single strain model with a result on the stability of endemic equilibrium when the whole habitat consists of only high-risk environment.

Theorem 2.8 Suppose that $k=1$ and $\mathfrak{R}_{0}>1$. Let $\left(S_{e}(x), I_{e}(x)\right)$ be the unique endemic equilibrium given by Theorem 2.6 (ii). Then given any positive initial data $(S(0, x), I(0, x))$, its corresponding unique classical solution $(S(t, x), I(t, x))$ of $(1)$ satisfies

$$
\lim _{t \rightarrow \infty}\left(\left\|S(t, \cdot)-S_{e}(\cdot)\right\|_{\infty}+\left\|I(t, \cdot)-I_{e}(\cdot)\right\|_{\infty}\right)=0
$$

Thanks to Theorems $2.5,2.6 \& 2.8$, if $\Re_{0}<1$ and $d_{\mathrm{S}}=0$, then the singlestrain disease will be eradicated and there is no endemic equilibrium. However, if $\mathfrak{R}_{0}>1$, then the single-strain disease will become permanent and every solution will converge to the unique endemic equilibrium. Hence, the quantity $\mathfrak{R}_{0}$, which is independent of the diffusion rate $d_{1}$ of the infected subgroup, plays the role of the basic reproduction number of (1) when $d_{\mathrm{S}}=0$. For each $i=1, \ldots, k$, it is well known that $\mathcal{R}_{0, i} \geq \frac{\int_{\Omega} \beta_{i}}{\int_{\Omega} \gamma_{i}}$ for every $d_{i}>0$, and equality holds if and only if $\Re_{i}$ is constant. Hence, for example when $k=1$, it holds that $\mathfrak{R}_{0} \leq \inf _{d_{1}>0} \mathcal{R}_{0,1}$, and equality holds if and only if $\mathfrak{R}_{1}$ is constant. This shows that when the local reproduction rates are space homogeneous then a total lock-down of the susceptible host helps in lowering the disease' reproduction number.

We complete the list of our results with a result on the existence of coexistence EE when $H^{-}$is empty, $d_{\mathrm{S}}=0$ and $k=2$.

Theorem 2.9 Suppose that $k=2, \mathfrak{R}_{0}>1$ and both $\Sigma_{1}$ and $\Sigma_{2}$ are nonempty. Let $D_{1}$ and $D_{2}$ be two positive real numbers and set $d_{i}=\varepsilon D_{i}$ for each $i=1,2$. Then there is $\tilde{\varepsilon}^{*}>0$ such that for every $0<\varepsilon<\tilde{\varepsilon}^{*}$, (1) has a coexistence EE, denoted by $\left(S^{*}(\cdot, \varepsilon), I_{1}^{*}(\cdot, \varepsilon), I_{2}^{*}(\cdot, \varepsilon)\right)$. Moreover, if $\frac{\beta_{1}}{\beta_{2}}$ is constant, then $\left(S^{*}(\cdot, \varepsilon), I_{1}^{*}(\cdot, \varepsilon), I_{2}^{*}(\cdot, \varepsilon)\right)$ is the only coexistence EE solution of (1) for every $0<\varepsilon<\tilde{\varepsilon}^{*}$.

We note from Theorem 2.9 that in the event of a complete lock-down of the susceptible hosts and absence of a common safety zone, the multi-strain disease may persist even if each of its strains has a nonempty low risk area. 


\section{Discussion}

In the current work, we study a system of nonlinear partial differential equations modeling the dynamics of a multi-strain infectious epidemic disease. We consider a finite number of strains $(k \geq 2)$ of the infectious disease and allow the recovery and infection rates for each strain to be spatially heterogeneous. Our multi-strain PDE-SIS epidemic model is a generalization of the single-strain PDE-SIS model introduced in Allen et al. (2008). We study the disease dynamics in both full model (by allowing all the diffusion rates to be positive) and the degenerate ODE-PDE model (by limiting the diffusion rate $d_{\mathrm{S}}$ of the susceptible subgroup $S$ to zero). Our epidemic model with $d_{\mathrm{S}}=0$ models the situation of a total lock-down of the susceptible host. We allow the diffusion rate of each infected subgroup to be positive. One reason for such choice of the diffusion rates is to permit the infected members of the population to seek for medical treatment by allowing them some degree of freedom in their movement, while ordering a total stay at home of the non-infected population.

When $d_{\mathrm{S}}>0$, we define the basic reproduction number $\mathcal{R}_{0}$ of the multi-strain PDESIS model to be the maximum of the basic reproduction numbers $\mathcal{R}_{0, i}, i=1, \ldots, k$, of the single-strain PDE-SIS epidemic models. Since each of the single-strain basic reproduction number is independent of the susceptible host diffusion rate $d_{\mathrm{S}}$, then $\mathcal{R}_{0}$ is independent of $d_{\mathrm{S}}$ as well. We then show in Theorem 2.1 that all the strains of the infectious disease will be controlled and be eradicated in the long run if the basic reproduction number $\mathcal{R}_{0}$ is less or equal to one. However, if the reproduction number $\mathcal{R}_{0}$ is larger than one, then the disease will become permanent in the population. Then we seek to understand which of the strains of the disease will persist if $\mathcal{R}_{0}$ is bigger than one. In Theorem 2.2, we show that when the whole population has equal diffusion rate and the disease' local basic reproduction functions are positive constant numbers, then only the strain with the largest reproduction number can persist while all the remaining strains will go extinct in the long run. This phenomenon is known as the competition exclusion principle for different pathogens of the same disease as mentioned before. Figure 1 shows simulations of this competition exclusion principle for two- and three-strain models.

As the diffusion rate $d_{\mathrm{S}}>0$ of the susceptible host does not affect the permanence of the infectious disease, it is natural to ask whether reducing the size of $d_{\mathrm{S}}$ would lower the impact of the disease on the population once it is permanent. To this end, we study the asymptotic limit of endemic equilibrium solutions of the fully parabolic multi-strain PDE-SIS model as $d_{\mathrm{S}}$ tends to zero. Our result (Theorem 2.3) shows that if there is a common safety region against all the strains of the disease, then the total size of the infected subgroup of the population at endemic equilibrium is proportional to the susceptible host diffusion rate $d_{\mathrm{S}}$ as $d_{\mathrm{S}}$ tends to zero. On the other hand, Theorem 2.4 shows that the later result is false when there is no common safety region against all the strains. These results suggest that reducing the susceptible host movement is an effective control strategy to lower the impact of a disease on a population when there is a common safety area against all the strains. Figure 2 shows simulations illustrating the permanence of more than one strain of the disease when there is no common safety region against all the strains even though each strain has a nonempty safety area. 


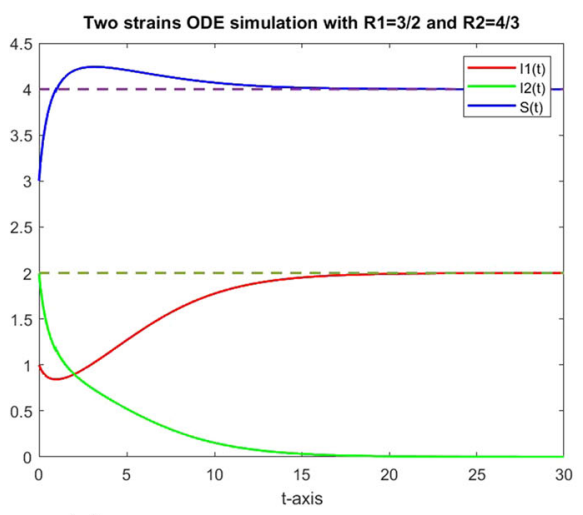

(a) Simulation for two-strain ODE model.

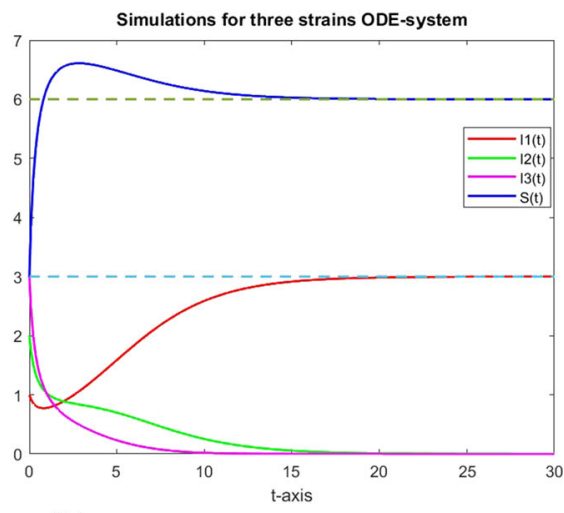

(b) Simulations for three-strain ODE model.

Fig. 1 a Simulations of solutions for two-strain $\operatorname{ODE}$ system with $\left(\beta_{1}, \gamma_{1}\right)=(3,2)$ and $\left(\beta_{2}, \gamma_{2}\right)=$ $(4,3)$ and initial $\left(S(0), I_{1}(0), I_{2}(0)\right)=(3,1,2)$. Hence, $N=6$ and $\left(S_{1}^{*}, I_{1,1}^{*}, I_{2,1}^{*}\right)=(4,2,0)$ and $\left(S_{2}^{*}, I_{1,2}^{*}, I_{2,2}^{*}\right)=\left(\frac{9}{2}, 0, \frac{3}{2}\right)$ are the two single-strain EE solutions. The simulation shows that strain one outcompetes strain two and drives it to extinction. $\mathbf{b}$ Simulations of solutions for three-strain ODE system with $\left(\beta_{1}, \gamma_{1}\right)=(3,2),\left(\beta_{2}, \gamma_{2}\right)=(4,3)$, and $\left(\beta_{3}, \gamma_{3}\right)=(5,4)$ and initial $\left(S(0), I_{1}(0), I_{2}(0), I_{3}(0)\right)=$ $(3,1,2,3)$. Hence, $N=9$ and $\left(S_{1}^{*}, I_{1,1}^{*}, I_{2,1}^{*}, I_{3,1}^{*}\right)=(6,3,0,0),\left(S_{2}^{*}, I_{1,2}^{*}, I_{2,2}^{*}, I_{3,2}^{*}\right)=\left(\frac{27}{4}, 0, \frac{8}{4}, 0\right)$, and $\left(S_{3}^{*}, I_{1,3}^{*}, I_{2,3}^{*}, I_{3,3}^{*}\right)=\left(\frac{36}{5}, 0,0, \frac{9}{5}\right)$ are the three single-strain EE solutions. The simulation shows that strain one drives strains two and three to extinction

To better understand how the movement of the susceptible host impacts the spread of the disease, we also study the dynamics of the multi-strain PDE-SIS model with $d_{\mathrm{S}}=0$, i.e., there is a total lock-down of the susceptible host. In the case of the single-strain model, our results in Theorems 2.5 and 2.8 provide a fairly complete picture of the dynamics. These results show that the outcome of the disease depends delicately on the local basic reproduction number $\mathfrak{R}_{1}$ for the single-strain model. In particular, it follows from Theorems 2.5 and 2.8 that for the single-strain model and a total lock-down of the susceptible host, the existence of a low risk area for all the strains of the disease is enough to eradicate the disease in the long run. However if the whole habitat consists of only high-risk area to the disease, then the disease will persist and the population will stabilize at the unique endemic equilibrium. In view of these results, we may conclude that a total lock-down of the susceptible host is a good control strategy leading to the eradication of the disease only if some additional safety measures are implemented to create a safety area for the disease. Simulations in Figs. 3 and 4 confirm these theoretical results. An interpretation of these results is that even in the event of a total lock-down of the susceptible host during the outbreak of a multi-strain infectious disease, it is indispensable to create a common safety area for all the strains if one wishes to eradicate the disease.

Acknowledgements We thank the editor and the anonymous referees for their comments and suggestions which help improve the presentation of the paper. YL is partially supported by NICE Visiting Program and NSF grant DMS-1853561. 


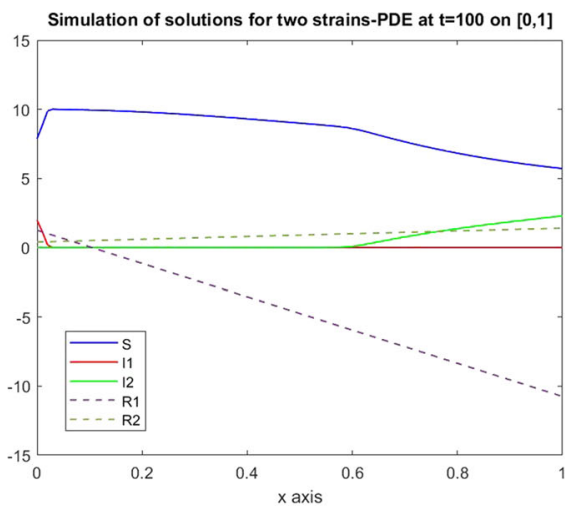

(a) Simulation of solutions with $H^{-} \neq \emptyset$.
Simulation of solutions for two strains-PDE at $t=100$ on $[0,1]$

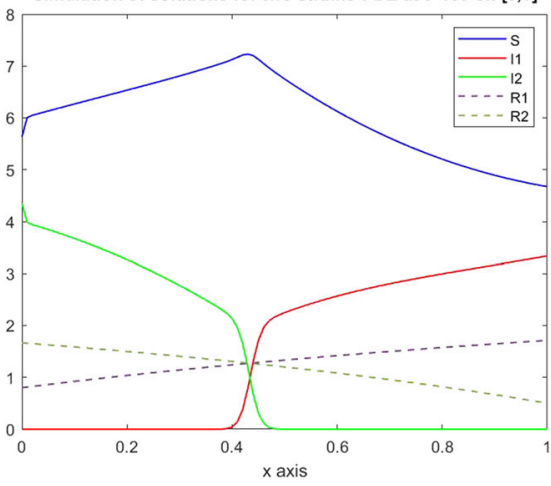

(b) Simulations of solutions with $H^{-}=\emptyset$.

Fig. 2 a Simulations of solutions at time $t=100$ for two-strain PDE-SIS system with $\gamma_{1}=\gamma_{2}=1$, $\beta_{1}(x)=1.25-12 x$ and $\beta_{2}(x)=0.4+x$ on $\Omega=[0,1]$. The initial data are $S(0, x)=2+\cos (\pi x)$, $I_{1}(0, x)=3+\cos (\pi x)$, and $I_{2}(0, x)=4-\cos (\pi x)$. Hence, $R 1:=\Re_{1}=\beta_{1}$ and $R 2:=\Re_{2}=\beta_{2}$. The diffusion rates are $d_{\mathrm{S}}=10^{-11}$ and $d_{1}=d_{2}=10^{-6}$. The graphs of $R 1$ and $R 2$ show that the common safety area, $H^{-}=(0.02,0.6)$, against two strains is nonempty. The simulations $I 1(x):=I_{1}(100, x)$ and $I 2(x):=I_{2}(100, x)$ are for steady states of Theorem 2.3. b Simulations of solutions at time $t=100$ for two-strain PDE-SIS system with $\beta_{1}(x)=2+4 x, \gamma_{1}(x)=2.5+x, \beta_{2}(x)=5-4 x$ and $\gamma_{2}(x)=3-x$ with initial data $S(0, x)=2+\cos (\pi x), I_{1}(0, x)=3+\cos (\pi x)$, and $I_{2}(0, x)=4-\cos (\pi x)$. The diffusion rates are all equal, $d_{\mathrm{S}}=d_{1}=d_{2}=0.0001$. The graphs of $R 1=\mathfrak{R}_{1}$ and $R 2=\mathfrak{R}_{2}$ are also given. The simulations show that both strains persist and that each strain drives the other to extinction on the set where its local basic reproduction function is the largest. Both $H_{1}^{-}$and $H_{2}^{-}$are non-empty sets and that $H^{-}=\emptyset$. The simulations $I 1(x)=I_{1}(100, x)$ and $I 2(x)=I_{2}(100, x)$ are for steady states of Theorem 2.4

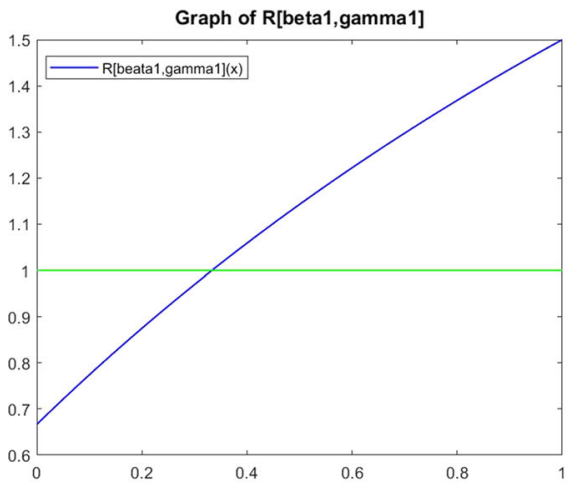

(a) Graph of $\mathfrak{R}_{1}$

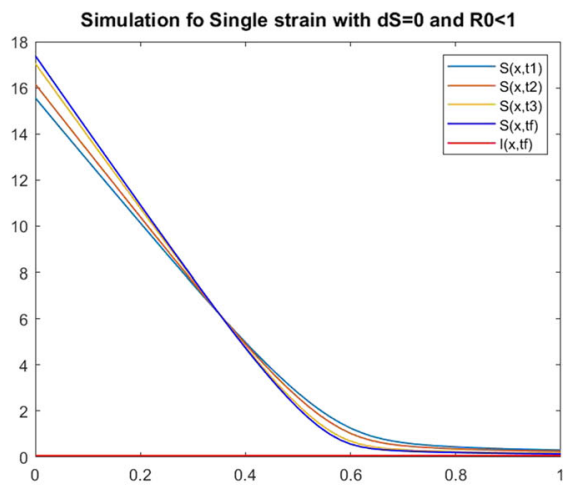

(b) Simulation of solutions.

Fig. 3 Illustration of Theorem 2.5. Simulations of solutions of (1) on $\Omega=(0,1)$ with the choice of parameters: $d_{\mathrm{S}}=0, d_{1}=1, \beta_{1}(x)=2+4 x, \gamma_{1}=3+x$. We take the initial data $(S(0, x), I(0, x))=$ $(3+\cos (\pi x), 2+\cos (\pi x))$, hence $N=5$. The graph in (a) is the graph of $\Re_{1}$. The graphs in $(\mathbf{b})$ are simulations of the solutions at different times

\section{Appendix A: Proofs of Theorems 2.1, 2.2 and 2.3}

This section is devoted to the proofs of Theorems 2.1, 2.2 and 2.3. First, we derive some estimates on solutions. Let us denote by $\left\{e^{t \Delta}\right\}_{t \geq 0}$ the heat-semigroup on $L^{p}(\Omega)$, 


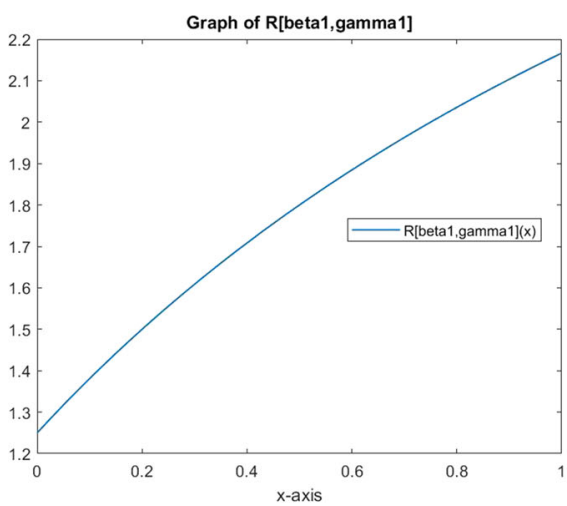

(a) Graph of $\Re_{1}(x)$

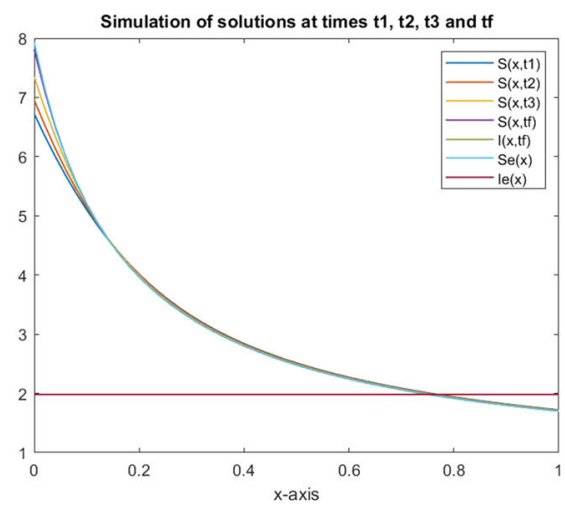

(b) Simulation of solutions.

Fig. 4 Illustration of Theorem 2.9. Simulations of solutions of (1) on $\Omega=(0,1)$ with the choice of parameters: $d_{\mathrm{S}}=0, d_{1}=1, \beta_{1}(x)=2.5+4 x, \gamma_{1}=2+x .(S(0, x), I(0, x))=(3+\cos (\pi x), 2+\cos (\pi x))$, hence $N=5$. The graph in (a) is the graph of $\Re_{1}$. The graphs in (b) are simulations of the solutions at different times. We see from Fig. (b) that the graphs of the simulations $S(t, \cdot)$ converge to the graph of $S_{e}(x)$ uniformly in $x \in[0,1]$

$p>n$ subject to no-flux condition on $\partial \Omega$. It follows from (Quittner and Souplet 2007, Proposition $48.4^{*}$-(f)) that there is a positive constant $C_{*}$ depending on $\Omega$ and the space dimension $n$ such that

$$
\left\|e^{t \Delta} \phi\right\|_{\infty} \leq\left[C_{*} \min \{t, 1\}\right]^{-\frac{n}{2}}\|\phi\|_{L^{1}(\Omega)} \quad \forall \phi \in L^{1}(\Omega) .
$$

For convenience, for $f=\beta$ or $f=\gamma$, we let

$$
\underline{f}=\min \left\{f_{i}(x): x \in \bar{\Omega}, i=1, \ldots, k\right\} \text { and } \bar{f}=\max \left\{\left\|f_{i}\right\|_{\infty}: i=1, \ldots, k\right\} .
$$

Let $k \geq 1$ be fixed and $\left(S(t, x), I_{1}(t, x), \ldots, I_{k}(t, x)\right)$ be a classical solution of (1). Observe that for each $i=1, \ldots, k, I_{i}(t, x)$ satisfies

$$
\left\{\begin{array}{l}
\partial_{t} I_{i}=d_{i} \Delta I_{i}+F_{i}(t, x) I_{i} \quad x \in \Omega, t>0 \\
0=\partial_{\vec{n}} I_{i} \quad x \in \partial \Omega, t>0
\end{array}\right.
$$

with $F_{i}=\beta_{i} \frac{S}{S+\sum_{j=1}^{k} I_{j}}-\gamma_{i}$ satisfying $\left\|F_{i}\right\|_{\infty} \leq\left\|\beta_{i}+\gamma_{i}\right\|_{\infty} \leq \bar{\beta}+\bar{\gamma}<\infty$. Note also that

$$
N=\int_{\Omega}\left(S(t, x)+\sum_{i=1}^{k} I_{i}(t, x)\right) \mathrm{d} x \quad \forall t \geq 0
$$


since assumption (A) holds and that

$$
\frac{\mathrm{d}}{\mathrm{d} t} \int_{\Omega}\left[S(t, \cdot)+\sum_{i=1}^{k} I_{i}(t, \cdot)\right]=\int_{\Omega}\left[d_{\mathrm{S}} \Delta S+\sum_{i=1}^{k} d_{i} \Delta I_{i}\right]=0 \quad \forall t>0
$$

Hence, by (11), (12), (13) and the comparison principle for parabolic equations, we have

$$
\left\|I_{i}\left(t+t_{0}, \cdot\right)\right\|_{\infty} \leq e^{t(\bar{\beta}+\bar{\gamma})}\left\|e^{t d_{i} \Delta} I_{i}\left(t_{0}, \cdot\right)\right\|_{\infty} \leq e^{t(\bar{\beta}+\bar{\gamma})}\left[C_{*} \min \left\{t d_{i}, 1\right\}\right]^{-\frac{n}{2}} N
$$

for every $t>0, t_{0}>0$ and $i=1, \ldots, k$. Therefore, letting $c_{t_{0}}=\sum_{i=1}^{k} c_{t_{0}, i}$ where $c_{t_{0}, i}=e^{\frac{t_{0}}{2}(\bar{\beta}+\bar{\gamma})}\left[C_{*} \min \left\{\frac{d_{i} t_{0}}{2}, 1\right\}\right]^{-\frac{n}{2}}$ for every $t_{0}>0$ and $i=1, \ldots, k$, we conclude that

$$
\sum_{i=1}^{k}\left\|I_{i}(t, \cdot)\right\|_{\infty} \leq c_{t_{0}} N \quad \forall t \geq t_{0}, \quad d_{\mathrm{S}} \geq 0
$$

Moreover, the regularity theory for parabolic equations (Henry (1981)) yields that the map

$[1, \infty) \ni t \mapsto I_{i}(t, \cdot) \in C^{1+\theta}(\Omega)$ is continuous (for every $d_{\mathrm{S}} \geq 0$ ), $i=1, \ldots, k$,

for $0<\theta \ll 1$. Note also that since $I_{i}$ satisfies (12) for each $i$, by the Harnack inequality for linear parabolic equations (Húska 2006, Theorem 2.1), for every $t_{0}>0$ there is $\tilde{c}_{t_{0}}$ independent of $d_{\mathrm{S}}$ such that

$$
I_{i}(t, x) \leq \tilde{c}_{t_{0}} I_{i}(t, y) \quad \forall t \geq t_{0}, x, y \in \Omega, \forall d_{\mathrm{S}} \geq 0, i=1, \ldots, k .
$$

Now suppose that $d_{\mathrm{S}}>0$ and observe from (14) and the equation satisfied by $S(t, x)$ that

$$
\partial_{t} S \leq d_{\mathrm{S}} \Delta S+c_{1} N \bar{\gamma} \quad t \geq 1, x \in \Omega .
$$

Thus, by the comparison principle for parabolic equations and recalling inequality (11),

$$
\begin{aligned}
S(1+t) & \leq e^{d_{\mathrm{S}} \Delta} S(t)+\int_{0}^{1} e^{(1-s) d_{\mathrm{S}} \Delta}\left(c_{1} N \bar{\gamma}\right) \mathrm{d} s \leq e^{d_{\mathrm{S}} \Delta} S(t)+c_{1} N \bar{\gamma} \\
& \leq \frac{\|S(t)\|_{L^{1}}}{\left[C_{*} \min \left\{d_{\mathrm{S}}, 1\right\}\right]^{\frac{n}{2}}}+c_{1} N \bar{\gamma} \leq \frac{N}{\left[C_{*} \min \left\{d_{\mathrm{S}}, 1\right\}\right]^{\frac{n}{2}}}+c_{1} N \bar{\gamma} \quad \forall t>0 .
\end{aligned}
$$

The following result shows that the susceptible host $S(t, x)$ persists uniformly in $x \in \Omega$. 
Lemma 3.1 There is a positive constant $C^{*}$, depending only on $\Omega$, such that

$$
S\left(t+t^{\prime}, x\right) \geq \frac{C^{*} e^{-t \bar{\beta}}}{|\Omega|}\left\{\int_{\Omega} S(0, x)+\frac{\underline{\gamma} N}{\underline{\gamma}+\bar{\beta}}\left(1-e^{-(\underline{\gamma}+\bar{\beta}) t^{\prime}}\right)\right\}, \quad t^{\prime}>0, t \geq \frac{1}{d_{\mathrm{S}}}, \quad x \in \Omega .
$$

Hence,

$$
\liminf _{t \rightarrow \infty} \min _{x \in \bar{\Omega}} S(t, x) \geq M^{*}:=\frac{\underline{\gamma} N C^{*} e^{-\frac{\bar{\beta}}{d_{\mathrm{S}}}}}{|\Omega|(\underline{\gamma}+\bar{\beta})}
$$

Proof First, observe from (13) that

$$
\frac{\mathrm{d}}{\mathrm{d} t} \int_{\Omega} S=\int_{\Omega} \sum_{i=1}^{k} \gamma_{i} I_{i}-\int_{\Omega} S \frac{\sum_{i=1}^{k} \beta_{i} I_{i}}{S+\sum_{i=1}^{k} I_{i}} \geq \underline{\gamma} \int_{\Omega} \sum_{i=1}^{k} I_{i}-\bar{\beta} \int_{\Omega} S=N \underline{\gamma}-(\underline{\gamma}+\bar{\beta}) \int_{\Omega} S .
$$

Hence, by the comparison principle for ODE,

$$
\int_{\Omega} S(t, x) \mathrm{d} x \geq \int_{\Omega} S(0, x) \mathrm{d} x+\frac{\underline{\gamma} N}{\underline{\gamma}+\bar{\beta}}\left(1-e^{-(\underline{\gamma}+\bar{\beta}) t}\right) \quad \forall t>0 .
$$

Next, since

$$
\partial_{t} S(t) \geq d_{\mathrm{S}} \Delta S-\left\|\frac{\sum_{i=1}^{k} \beta_{i} I_{i}}{S+\sum_{j=1}^{k} I_{j}}\right\|_{\infty} S \geq d_{\mathrm{S}} \Delta S-\bar{\beta} S, \quad \forall t>0,
$$

then

$$
S\left(t+t_{0}\right) \geq e^{-t \bar{\beta}} e^{t d_{\mathrm{S}} \Delta} S\left(t_{0}\right) \quad \forall t>0, t_{0}>0,
$$

by the comparison principle for parabolic equations. Recalling the Harnack inequality (Húska 2006, Theorem 2.5), we know that there is a positive constant $C^{*}$ depending on $\Omega$ such that

$$
\left(e^{t \Delta} \phi\right)(x) \geq C^{*}\left(e^{t \Delta} \phi\right)(y) \quad \forall t \geq 1, y, x \in \Omega, \phi \in C^{+}(\bar{\Omega}),
$$

which together with (19) yield that

$$
S\left(t+t_{0}, x\right) \geq \frac{C^{*}}{|\Omega|} e^{-\bar{\beta} t}\left\|S\left(t_{0}\right)\right\|_{L^{1}(\Omega)}, \quad t_{0}>0, t \geq \frac{1}{d_{\mathrm{S}}}, x \in \Omega .
$$

Whence, in view of (18), we deduce the assertion of the lemma. 
Next, for each $i=1, \ldots, k$, let $\lambda^{*}\left(d_{i}, \beta_{i}-\gamma_{i}\right)$ denote the principal eigenvalue of the linear elliptic eigenvalue problem

$$
\begin{cases}0=d_{i} \Delta \psi+\left(\beta_{i}-\gamma_{i}\right) \psi+\lambda \psi & x \in \Omega \\ \frac{\partial \psi}{\partial \vec{n}}=0 & x \in \partial \Omega\end{cases}
$$

It is well known that $1-\mathcal{R}_{0, i}$ and $\lambda^{*}\left(d_{i}, \beta_{i}-\gamma_{i}\right)$ have the same sign (see Allen et al. 2008). Thanks to (16) and Lemma 3.1, we can present a proof of Theorem 2.1.

Proof of Theorem 2.1 (i) Let $i \in\{1, \ldots, k\}$ such that $\mathcal{R}_{0, i} \leq 1$ and $\lambda_{i}^{*}:=\lambda^{*}\left(d_{i}, \beta_{i}-\right.$ $\gamma_{i}$ ) be the eigenvalue of (20). Let $\psi_{i}^{*}$ be the corresponding eigenfunction satisfying $\int_{\Omega} \psi_{i}^{*}=1$. Multiplying the equation satisfied by $I_{i}(t, x)$ by $\psi_{i}^{*}$ and integrate it by parts on $\Omega$, we get

$$
\frac{\mathrm{d}}{\mathrm{d} t} \int_{\Omega} I_{i} \psi_{i}^{*}=-d_{i} \int_{\Omega} \nabla \psi_{i}^{*} \nabla I_{i}+\int_{\Omega}\left(\beta_{i}-\gamma_{i}\right) \psi_{i}^{*} I_{i}-\int_{\Omega} \beta_{i} \frac{\sum_{j=1}^{k} I_{j}}{S+\sum_{j=1}^{k} I_{j}} \psi_{i}^{*} I_{i} .
$$

Multiplying the equation of $\left(\lambda_{i}^{*}, \psi_{i}^{*}\right)$ by $I_{i}$ and integrate it by parts on $\Omega$ yield

$$
-\lambda_{i}^{*} \int_{\Omega} I_{i} \psi_{i}^{*}=-d_{i} \int_{\Omega} \nabla \varphi_{i} \nabla I_{i}+\int_{\Omega}\left(\beta_{i}-\gamma_{i}\right) \psi_{i}^{*} I_{i}
$$

Hence, by (21),

$$
\frac{\mathrm{d}}{\mathrm{d} t} \int_{\Omega} I_{i} \psi_{i}^{*}=-\lambda_{i}^{*} \int_{\Omega} I_{i} \psi_{i}^{*}-\int_{\Omega} \beta_{i} \frac{\sum_{j=1}^{k} I_{j}}{S+\sum_{j=1}^{k} I_{j}} \psi_{i}^{*} I_{i}
$$

We integrate this equation to get

$$
\int_{\Omega} I_{i}(0) \psi_{i}^{*}=e^{\lambda_{i}^{*} t} \int_{\Omega} I_{i}(t) \psi_{i}^{*}+\int_{0}^{t} \int_{\Omega} \beta_{i} e^{\lambda_{i} s} \frac{\sum_{j=1}^{k} I_{j}}{S+\sum_{j=1}^{k} I_{j}} \psi_{i}^{*} I_{i}
$$

Recalling inequalities (14) and (17), there is some positive constant $M$ such that $S(t, x)+\sum_{i=1}^{k} I_{i}(t, x) \leq M$ for every $t \geq 0$ and $x \in \Omega$, and hence

$$
\begin{aligned}
\int_{0}^{t} \int_{\Omega} \frac{e^{\lambda_{i}^{*} s} \beta_{i} \sum_{j=1}^{k} I_{j}}{S+\sum_{j=1}^{k} I_{j}} \psi_{i}^{*} I_{i}^{*} \mathrm{~d} x \mathrm{~d} s & \geq \frac{1}{M} \int_{0}^{t} \int_{\Omega} e^{\lambda_{i}^{*} s} \beta_{i} \psi_{i}^{*} I_{i} \sum_{i=1}^{k} I_{j} \mathrm{~d} x \mathrm{~d} s \\
& \geq \frac{\min _{x \in \bar{\Omega}} \beta_{i}(x) \psi_{i}^{*}(x)}{M} \int_{0}^{t} e^{\lambda_{i}^{*} s}\left\|I_{i}(s)\right\|_{L^{2}(\Omega)}^{2} \mathrm{~d} s
\end{aligned}
$$


for every $t>0$. Hence, in view of (23), we get

$$
\int_{\Omega} I_{i}(0) \psi_{i}^{*} \geq e^{\lambda_{i}^{*} t} \min _{x \in \bar{\Omega}} \psi_{i}^{*}(x)\left\|I_{i}(t)\right\|_{L^{1}(\Omega)}+\frac{\min _{x \in \bar{\Omega}} \beta_{i}(x) \psi_{i}^{*}(x)}{M} \int_{0}^{t} e^{\lambda_{i}^{*} s}\left\|I_{i}(s)\right\|_{L^{2}(\Omega)}^{2} \mathrm{~d} s .
$$

Since $\lambda_{i}^{*} \geq 0$, we then conclude from (11), (15), and (24) that $\left\|I_{i}(t)\right\|_{\infty} \rightarrow 0$ as $t \rightarrow \infty$.

(ii) Suppose that $\mathcal{R}_{0} \leq 1$. By $(i)$, we know that $\max _{1 \leq i \leq k}\left\|I_{i}\right\|_{\infty} \rightarrow 0$ as $t \rightarrow \infty$. We can then proceed as in Allen et al. (2008) to derive the desired result.

(iii) Suppose that $\mathcal{R}_{0}>1$ and let $J_{0}=\left\{i \in\{1, \ldots, k\}: \mathcal{R}_{0, i}>1\right\}$. Hence $\lambda_{*}:=\min \left\{-\lambda^{*}\left(d_{i}, \beta_{i}-\gamma_{i}\right): i \in J_{0}\right\}>0$. By Lemma 3.1, there is $\underline{m}_{*}>0$ such that $\underline{m}_{*} \leq S(t, x)$ for every $x \in \Omega$ and $t \geq 1$. Taking $\underline{n}_{*}=\min _{i \in J_{0}, x \in \bar{\Omega}} \psi_{i}^{*}(x)$ and using (22), we obtain that

$$
\begin{aligned}
& \frac{\mathrm{d}}{\mathrm{d} t} \sum_{i \in J_{0}}\left\|\psi_{i}^{*} I_{i}\right\|_{L^{1}(\Omega)} \\
& \quad \geq \lambda_{*} \sum_{i \in J_{0}}\left\|\psi_{i}^{*} I_{i}\right\|_{L^{1}(\Omega)}-\frac{\bar{\beta}}{\underline{m}_{*}} \int_{\Omega}\left(\sum_{j \notin J_{0}} I_{j}+\frac{1}{\underline{n}_{*}} \sum_{j \in J_{0}} \psi_{j}^{*} I_{j}\right) \sum_{i \in J_{0}} \psi_{i}^{*} I_{i} \\
& \quad \geq\left(\lambda_{*} \sum_{i \in J_{0}}\left\|\psi_{i}^{*} I_{i}\right\|_{L^{1}(\Omega)}-\frac{\bar{\beta}}{\underline{m}_{*}} \sum_{j \notin J_{0}}\left\|I_{j}\right\|_{\infty} \sum_{i \in J_{0}}\left\|\psi_{i}^{*} I_{i}\right\|_{L^{1}(\Omega)}-\frac{\bar{\beta}}{\underline{m}_{*} \underline{n}_{*}} \int_{\Omega}\left(\sum_{i \in J_{0}} \psi_{i}^{*} I_{i}\right)^{2}\right) \\
& \quad \geq\left(\lambda_{*}-\frac{\bar{\beta}}{\underline{m}_{*}} \sum_{j \notin J_{0}}\left\|I_{j}\right\|_{\infty}-\frac{\bar{\beta}}{\underline{m}_{*} \underline{n}_{*}|\Omega|} \sum_{i \in J_{0}}\left\|\psi_{i}^{*} I_{i}\right\|_{L^{1}(\Omega)}\right) \sum_{i \in J_{0}}\left\|\psi_{i}^{*} I_{i}\right\|_{L^{1}(\Omega)}, \quad t>1 .
\end{aligned}
$$

But by $(i)$, we know that $\sum_{j \notin J_{0}}\left\|I_{j}\right\|_{\infty} \rightarrow 0$ as $t \rightarrow \infty$. It then follows from the last inequality that

$$
\liminf _{t \rightarrow \infty} \sum_{i \in J_{0}}\left\|\psi_{i}^{*} I_{i}\right\|_{L^{1}(\Omega)} \geq \frac{\lambda_{*} \underline{m}_{*} \underline{n}_{*}}{\bar{\beta}}
$$

which together with (16) yields the desired result.

Next, we present a proof of Theorem 2.2. We first introduce the functions

$$
\beta_{1, *}=\beta_{1}-\max _{2 \leq i \leq k} \beta_{i} \quad \text { and } \quad \gamma_{1, *}=\min _{2 \leq i \leq k} \gamma_{i}-\gamma_{1}
$$

Proof of Theorem 2.2 Suppose that $d=d_{1}=\cdots=d_{k}$.

(i) Suppose $\sigma_{1}^{*}=\min _{x \in \bar{\Omega}} \gamma_{1, *}(x)>0$ and $\beta=\beta_{1}=\cdots=\beta_{k}$. For every $i \geq 2$, we have

$$
\begin{cases}\left(e^{-\sigma_{1}^{*} t} I_{1}\right)_{t} \geq d \Delta\left(e^{-\sigma_{1}^{*} t} I_{1}\right)-\gamma_{i}\left(e^{-\sigma_{1}^{*} t} I_{1}\right)+\frac{\beta S}{S+\sum_{j} I_{j}}\left(e^{-\sigma_{1}^{*} t} I_{1}\right) & x \in \Omega, \\ \partial_{\vec{n}}\left(e^{-\sigma_{1}^{*} t} I_{1}\right)=0 & x \in \partial \Omega .\end{cases}
$$


Hence, since $\min _{x \in \bar{\Omega}} I_{1}(1, x)>0$, it follows from the comparison principle that

$$
M e^{-\sigma_{1} t} I_{1}(t, x) \geq I_{i}(t, x) \quad \forall x \in \Omega, t \geq 1
$$

for some $M \gg 1$. Thus, the result follows.

(ii) Suppose that $\sigma_{2}^{*}=\min _{x \in \bar{\Omega}} \beta_{1, *}(x)>0$ and $\gamma=\gamma_{1}=\cdots=\gamma_{k}$. For every $i \geq 2$ and $\tau>0$, we have

$$
\begin{cases}\left(e^{-\tau \sigma_{2}^{*} t} I_{1}\right)_{t} \geq d \Delta\left(e^{-\tau \sigma_{2}^{*} t} I_{1}\right)-\gamma\left(e^{-\tau \sigma_{2}^{*} t} I_{1}\right) & \\ +\frac{\beta_{i} S\left(e^{-\tau \sigma_{2}^{*} t} I_{1}\right)}{S+\sum_{j=1}^{k} I_{j}}+\sigma_{2}^{*}\left(\frac{S}{S+\sum_{j=1}^{k} I_{j}}-\tau\right) e^{-\tau \sigma_{2}^{*} t} I_{1} & x \in \Omega, \\ \partial_{\vec{n}}\left(e^{-\tau \sigma_{2}^{*} t} I_{1}\right)=0 & x \in \partial \Omega .\end{cases}
$$

By Lemma 3.1 and estimate (14), we know that $\inf _{t \geq 1, x \in \bar{\Omega}} \frac{S(t, x)}{S(t, x)+\sum_{j} I_{j}(t, x)}>0$. Hence, by choosing $0<\tau \ll 1$, we have that

$$
\begin{cases}\left(e^{-\tau \sigma_{2}^{*} t} I_{1}\right)_{t} \geq d \Delta\left(e^{-\tau \sigma_{2}^{*} t} I_{1}\right)-\gamma\left(e^{-\tau \sigma_{2}^{*} t} I_{1}\right)+\frac{\beta_{i} S}{S+\sum_{j} I_{j}}\left(e^{-\tau \sigma_{2}^{*} t} I_{1}\right) & x \in \Omega, \\ \partial_{\vec{n}}\left(e^{-\tau \sigma_{2}^{*} t} I_{1}\right)=0 & x \in \partial \Omega .\end{cases}
$$

Since $\min _{x \in \bar{\Omega}} I_{1}(1, x)>0$, it then follows from the comparison principle that

$$
M e^{-\tau \sigma_{2}^{*} t} I_{1}(t, x) \geq I_{i}(t, x) \quad \forall x \in \Omega, t \geq 1
$$

for some $M \gg 1$. Thus, the result follows.

(iii) Set $u_{i}=\frac{1}{\hat{\beta}_{i}} \ln I_{i}$. Then

$$
\left\{\begin{array}{l}
\partial_{t} u_{i}=d \Delta u_{i}+d \hat{\beta}_{i}\left|\nabla u_{i}\right|^{2}+\frac{g(x) S}{S+I}-\frac{\hat{\gamma}_{i}}{\hat{\beta}_{i}} h(x) \quad x \in \Omega, i=1 \cdots, k \\
\partial_{\vec{n}} u_{i}=0 \quad x \in \partial \Omega, i=1, \ldots, k
\end{array}\right.
$$

Setting $\hat{l}:=\min \left\{\frac{\hat{\gamma}_{j}}{\hat{\beta}_{j}}: j=2, \ldots, k\right\}>\frac{\hat{\gamma}_{1}}{\hat{\beta}_{1}}$ and $\underline{h}:=\min _{x \in \bar{\Omega}} h(x)>0$, and choosing $M \gg 1$ such that $u_{1}(0, x)+M \geq u_{i}(0, x)$ for every $x \in \Omega$ and $j=2, \ldots, k$, we get

$$
\begin{gathered}
\partial_{t}\left(u_{1}-\underline{h}\left(\hat{l}-\frac{\hat{\gamma}_{1}}{\hat{\beta}_{1}}\right) t+M\right) \geq d \Delta\left(u_{1}-\underline{h}\left(\hat{l}-\frac{\hat{\gamma}_{1}}{\hat{\beta}_{1}}\right) t+M\right) \\
\quad+d \hat{\beta}_{i}\left|\nabla\left(u_{1}-\underline{h}\left(\hat{l}-\frac{\hat{\gamma}_{1}}{\hat{\beta}_{1}}\right) t+M\right)\right|^{2}+\frac{g(x) S}{S+I}-\frac{\hat{\gamma}_{i}}{\hat{\beta}_{i}} h(x)
\end{gathered}
$$

for every $x \in \Omega$ and $t>0$ since $\hat{\beta}_{1}=\max \left\{\hat{\beta}_{j}: j=1, \ldots, k\right\}$. Since $\partial_{\vec{n}}\left(u_{1}-\right.$ $\left.\underline{h}\left(\hat{l}-\frac{\hat{\gamma}_{1}}{\hat{\gamma}_{1}}\right) t+M\right)=\partial_{\vec{n}} u_{1}=\partial_{\vec{n}} u_{i}=0$ for every $t>0$ and $i=2, \ldots, k$, then by the 
comparison principle for parabolic equations and the choice of $M$, we obtain

$$
u_{1}(t, x)-\underline{h}\left(\hat{l}-\frac{\hat{\gamma}_{1}}{\hat{\beta}_{1}}\right) t+M \geq u_{j}(t, x) \quad \forall x \in \Omega, t>0, j=2, \ldots, k .
$$

This is equivalent to

$$
e^{M} I_{1}(t, x) e^{-\underline{h}\left(\hat{l}-\frac{\hat{\gamma}}{\hat{\beta}_{1}}\right) t} \geq I_{j}(t, x) \quad \forall x \in \Omega, t>0, j=2, \ldots, k .
$$

Thus, we conclude that $\left\|I_{j}\right\|_{\infty} \rightarrow 0$ as $t \rightarrow \infty$ since $\sup _{t \geq 0}\left\|I_{1}(t)\right\|_{\infty}<\infty$ and $\underline{h}\left(\hat{l}-\frac{\hat{\gamma}_{1}}{\hat{\beta}_{1}}\right)>0$.

(iv) Now, we suppose that $d_{\mathrm{S}}=d, \mathfrak{R}_{i}$ is a constant function for each $i=1, \ldots, k$ with $\mathfrak{R}_{1}>R^{*}:=\max \left\{\mathfrak{R}_{j}: j=2, \ldots, k\right\}$. We set $Z(t, x):=\sum_{j=2}^{k} I_{j}(t, x)$,

$$
\begin{aligned}
\underline{I}_{1} & :=\liminf _{t \rightarrow \infty} \min _{x \in \bar{\Omega}} I_{1}(t, x), \\
\bar{I}_{1} & :=\limsup _{t \rightarrow \infty} \max _{x \in \bar{\Omega}} I_{1}(t, x), \text { and } \bar{Z}:=\limsup _{t \rightarrow \infty} \max _{x \in \bar{\Omega}} Z(t, x) .
\end{aligned}
$$

By recalling that

$$
\partial_{t}\left(S+\sum_{i=1}^{k} I_{i}\right)=d \Delta\left(S+\sum_{i=1}^{k} I_{i}\right) \quad x \in \Omega, t>0 \quad \text { and } \int_{\Omega}\left(S+\sum_{i=1}^{k} I_{i}\right)=N \forall t \geq 0
$$

and $\partial_{\vec{n}}\left(S+\sum_{i=1}^{k} I_{i}\right)=0$, then $S+\sum_{i=1}^{k} I_{i} \rightarrow \frac{N}{|\Omega|}$ as $t \rightarrow \infty$ uniformly on $\bar{\Omega}$. Hence, for every $0<\varepsilon \ll 1$, there is $t_{\varepsilon} \gg 1$ such that for each $i=1, \ldots, k$,

$$
\partial_{t} I_{i} \leq d \Delta I_{i}+\frac{\beta_{i}|\Omega|}{N(1+\varepsilon)}\left(\frac{N(1+\varepsilon)}{|\Omega|}\left(1-\frac{1}{\Re_{i}}\right)-\left(I_{1}+Z\right)\right) I_{i} \quad t>t_{\varepsilon}, x \in \Omega,
$$

$\partial_{t} I_{i} \geq d \Delta I_{i}+\frac{\beta_{i}|\Omega|}{N(1-\varepsilon)}\left(\frac{N(1-\varepsilon)}{|\Omega|}\left(1-\frac{1}{\mathfrak{R}_{i}}\right)-\left(I_{1}+Z\right)\right) I_{i} \quad t>t_{\varepsilon}, x \in \Omega$,

and

$$
I_{1}(t, x) \geq \underline{I}_{1}-\varepsilon \quad \forall x \in \Omega, t>t_{\varepsilon} .
$$

It follows from (25) and (27) that

$$
\left\{\begin{array}{l}
\partial_{t} Z \leq d \Delta Z+\frac{|\Omega|}{N(1+\varepsilon)}\left(\frac{N(1+\varepsilon)}{|\Omega|}\left(1-\frac{1}{R^{*}}\right)-\underline{I}_{1}+\varepsilon-Z\right) \sum_{j=2}^{k} \beta_{j} I_{j} \\
\quad \forall t>t_{\varepsilon}, x \in \Omega \\
0=\partial_{\vec{n}} Z \quad x \in \partial \Omega, t>t_{\varepsilon} .
\end{array}\right.
$$


Thus, since $\underline{\beta} Z \leq \sum_{i=2}^{k} \beta_{i} I_{i} \leq \bar{\beta} Z$, we conclude from the comparison principle that

$$
\bar{Z} \leq\left(\frac{N(1+\varepsilon)}{|\Omega|}\left(1-\frac{1}{R^{*}}\right)-\underline{I}_{1}+\varepsilon\right)_{+} \forall \varepsilon>0,
$$

and letting $\varepsilon \rightarrow 0$ yields that

$$
\bar{Z} \leq\left(\frac{N}{|\Omega|}\left(1-\frac{1}{R^{*}}\right)-\underline{I}_{1}\right)_{+},
$$

which together with (26) gives that

$$
\underline{I}_{1} \geq\left(\frac{N}{|\Omega|}\left(1-\frac{1}{\Re_{1}}\right)-\left(\frac{N}{|\Omega|}\left(1-\frac{1}{R^{*}}\right)-\underline{I}_{1}\right)_{+}\right)_{+}
$$

Now, we claim that $\left(\frac{N}{|\Omega|}\left(1-\frac{1}{R^{*}}\right)-\underline{I}_{1}\right)_{+}=0$. If this were false, then by (29) we would have

$$
\underline{I}_{1} \geq \frac{N}{|\Omega|}\left(\frac{1}{R^{*}}-\frac{1}{\Re_{1}}\right)+\underline{I}_{1}
$$

which is impossible since $\Re_{1}>R^{*}$. Thus, $\left(\frac{N}{|\Omega|}\left(1-\frac{1}{R^{*}}\right)-\underline{I}_{1}\right)_{+}=0$ and then $\bar{Z}=0$ and $\underline{I}_{1} \geq \frac{N}{|\Omega|}\left(1-\frac{1}{\mathfrak{R}_{1}}\right)$ by (28) and (29), respectively. On the other hand, it follows from (25) that $\bar{I}_{1} \leq \frac{N}{|\Omega|}\left(1-\frac{1}{\mathfrak{R}_{1}}\right)$. So, we have shown that $I_{1}(t, \cdot) \rightarrow \frac{N}{|\Omega|}\left(1-\frac{1}{\mathfrak{R}_{1}}\right)$ as $t \rightarrow \infty$ uniformly in $x \in \bar{\Omega}$. Next, recalling that $S+\sum_{i=1}^{k} I_{i} \rightarrow \frac{N}{|\Omega|}$ as $t \rightarrow \infty$ uniformly in $x \in \bar{\Omega}$, we then conclude that $\left(S, I_{1}, I_{2}, \ldots, I_{k}\right) \rightarrow\left(\frac{N}{|\Omega| \Re_{1}}, \frac{N}{|\Omega|}\left(1-\frac{1}{\Re_{1}}\right), 0, \ldots, 0\right)$ as $t \rightarrow \infty$ uniformly in $x \in \Omega$.

We end this section with the proof of Theorem 2.3.

Proof of Theorem 2.3 Suppose by contradiction that (5) is false. Then there is a sequence $\left\{d_{\mathrm{S}_{m}}\right\}_{m \geq 1}$ of positive numbers with $d_{\mathrm{S}_{m}} \rightarrow 0$ as $m \rightarrow \infty$ and a sequence of positive steady states $\left(S_{m}, I_{1, m}, \ldots, I_{k, m}\right)$ such that either

$$
\liminf _{m \rightarrow \infty} \sum_{i=1}^{k} \frac{d_{i}}{d_{\mathrm{S}_{m}}}\left\|I_{i, m}\right\|_{\infty}=\infty
$$

or

$$
\limsup _{m \rightarrow \infty} \min _{x \in \bar{\Omega}} \sum_{i=1}^{k} \frac{d_{i}}{d_{\mathrm{S}_{m}}} I_{i, m}(x)=0
$$


Observe that

$$
0=\Delta\left(d_{\mathrm{S}_{m}} S_{m}+\sum_{i=1}^{k} d_{i} I_{i, m}\right) \quad x \in \Omega \quad \text { and } \frac{\partial\left(d_{\mathrm{S}_{m}} S_{m}+\sum_{i=1}^{k} d_{i} I_{i, m}\right)}{\partial \vec{n}}=0 \quad \forall x \in \partial \Omega
$$

for every $m \geq 1$. Thus, for every $m \geq 1$, there is positive constant $\kappa_{m}$ such that

$$
d_{\mathrm{S}_{m}} S_{m}(x)+\sum_{i=1}^{k} d_{i} I_{i, m}(x)=\kappa_{m} \quad \forall x \in \Omega
$$

Now, we will show that there is a subsequence $\left\{m_{1}\right\}_{m \geq 1}$ of $\{m\}_{m \geq 1}$ and a positive number $N^{*}$ such that

$$
\lim _{m \rightarrow \infty} \frac{\kappa_{m_{1}}}{d_{\mathrm{S}_{m_{1}}}}=N^{*}
$$

We scale the variables as follows:

$$
\tilde{S}_{m}=\frac{d_{S_{m}} S_{m}}{\kappa_{m}} \quad \text { and } \quad \tilde{I}_{i, m}=\frac{d_{i} I_{i, m}}{\kappa_{m}} \quad \forall m \geq 1 .
$$

Hence, $\tilde{S}_{m}=1-\sum_{i=1}^{k} \tilde{I}_{i, m}$ for every $m \geq 1$ and the function $\tilde{I}_{m}:=\sum_{j=1}^{k} \tilde{I}_{j, m}$ satisfies

$$
\begin{cases}0=\Delta \tilde{I}_{m}+\frac{\left(1-\tilde{I}_{m}\right) \sum_{j=1}^{k} \frac{\beta_{j}}{d_{j}} \tilde{I}_{j, m}}{\left(1-\tilde{I}_{m}\right)+d_{S_{m}} \sum_{j=1}^{k} \frac{1}{d_{j}} \tilde{I}_{j, m}}-\sum_{j=1}^{k} \frac{\gamma_{j}}{d_{j}} \tilde{I}_{j, m} & x \in \Omega, \\ \frac{\partial \tilde{I}_{m}}{\partial \vec{n}}=0 & x \in \partial \Omega\end{cases}
$$

for every $m \geq 1$. Observe that $\left\|\tilde{I}_{m}\right\|_{\infty}<1$ and

$$
\left\|\frac{\left(1-\tilde{I}_{m}\right) \sum_{j=1}^{k} \frac{\beta_{j}}{d_{j}} \tilde{I}_{j, m}}{\left(1-\tilde{I}_{m}\right)+d_{S_{m}} \sum_{j=1}^{k} \frac{1}{d_{j}} \tilde{I}_{j, m}}-\sum_{j=1}^{k} \frac{\gamma_{j}}{d_{j}} \tilde{I}_{j, m}\right\|_{\infty} \leq \sum_{j=1}^{k} \frac{\left\|\gamma_{j}\right\|_{\infty}+\left\|\beta_{j}\right\|_{\infty}}{d_{j}}
$$

for every $m \geq 1$. Hence, by a priori estimates for elliptic equations, there is $\tilde{I}_{\infty} \in$ $C^{1}(\bar{\Omega})$ and a subsequence $\left\{\tilde{I}_{m_{1}}\right\}_{m \geq 1}$ of $\left\{\tilde{I}_{m}\right\}_{m \geq 1}$ such that $\tilde{I}_{m_{1}} \rightarrow \tilde{I}_{\infty}$ as $m \rightarrow \infty$ in $C^{1}(\bar{\Omega})$. We claim that

$$
0 \leq \tilde{I}_{\infty}(x)<1 \quad \forall x \in H^{-} .
$$


Suppose on the contrary that (36) is false. Thus, there is $x^{*} \in H^{-}$such that $\tilde{I}_{\infty}\left(x^{*}\right)=1$ because $\left\|\tilde{I}_{\infty}\right\|_{\infty} \leq 1$. Choose $0<r_{*} \ll 1$ such that $B\left(x^{*}, r^{*}\right) \subset H^{-}$. Observe from (35) that

$$
0 \leq \Delta \tilde{I}_{m}+\sum_{j=1}^{k} \frac{\left(\beta_{j}-\gamma_{j}\right)}{d_{j}} \tilde{I}_{j, m} \leq \Delta \tilde{I}_{m} \quad x \in H^{-}, \forall m \geq 1
$$

that is, $\tilde{I}_{m}$ is subharmonic on $H^{-}$. Hence,

$$
\tilde{I}_{m_{1}}\left(x^{*}\right) \leq \frac{1}{\left|B\left(x^{*}, r^{*}\right)\right|} \int_{B\left(x^{*}, r^{*}\right)} \tilde{I}_{m_{1}}(x) \mathrm{d} x \quad \forall m \geq 1
$$

Letting $m \rightarrow \infty$ and using the fact that $0 \leq \tilde{I}_{\infty}(x) \leq 1$ for every $x \in \Omega$ and the uniform continuity of $\tilde{I}_{\infty}$, we obtain that

$$
I_{\infty}(x)=1 \quad \forall x \in B\left(x^{*}, r^{*}\right) .
$$

Next, let $\varphi \in C_{c}^{\infty}\left(B\left(x^{*}, r^{*}\right)\right)$ such that $\varphi \geq 0$ and $\|\varphi\|_{\infty}>0$. Multiplying (35) by $\varphi$ and integrating by parts, we obtain

$$
0 \leq \int_{B\left(x^{*}, r^{*}\right)} \tilde{I}_{m_{1}} \Delta \varphi+\int_{B\left(x^{*}, r^{*}\right)} L(x) \varphi \tilde{I}_{m_{1}} \quad \forall m \geq 1
$$

where $L(x):=\max \left\{\frac{\beta_{j}(x)-\gamma_{j}(x)}{d_{j}}: 1 \leq j \leq k\right\}<0$ for every $x \in \bar{B}\left(x^{*}, r^{*}\right)$. Letting $m \rightarrow \infty$ in the previous inequality and recalling that $\int_{B\left(x^{*}, r^{*}\right)} \Delta \varphi=0$ and (37), we get

$$
0 \leq \int_{B\left(x^{*}, r^{*}\right)} L(x) \varphi(x) d x
$$

which is impossible. Therefore, we conclude that (36) must hold. Now, observe that

$$
\begin{aligned}
N & =\int_{\Omega}\left(S_{m_{1}}+\sum_{j=1}^{k} I_{j, m_{1}}\right) \\
& =\frac{\kappa_{m_{1}}}{d_{\mathrm{S}_{m_{1}}}}\left(\int_{\Omega}\left(1-\tilde{I}_{m_{1}}\right)+\int_{\Omega} \sum_{j=1}^{k} \frac{d_{S_{m_{1}}}}{d_{j}} \tilde{I}_{j, m_{1}}\right) \quad \forall m \geq 1 .
\end{aligned}
$$


Since $0<\left\|\tilde{I}_{j, m}\right\|_{\infty} \leq 1$ for every $j=1, \ldots, k$, then $\int_{\Omega} \sum_{j=1}^{k} \frac{d_{\mathrm{s}_{m_{1}}}}{d_{j}} \tilde{I}_{j, m_{1}} \rightarrow 0$ as $m \rightarrow \infty$. We also note from the definition of $\tilde{I}_{\infty}$ and (36) that

$$
\lim _{m \rightarrow \infty} \int_{\Omega}\left(1-\tilde{I}_{m_{1}}\right)=\int_{\Omega}\left(1-\tilde{I}_{\infty}\right) \in(0,|\Omega|) .
$$

Hence, letting $m \rightarrow \infty$ in (38), we derive (34) with $N^{*}=\frac{N}{\int_{\Omega}\left(1-\tilde{I}_{\infty}\right)} \in(0, \infty)$.

By (34) and the fact that $\left\|\tilde{I}_{i, m_{1}}\right\|_{\infty} \leq 1$ for every $m \geq 1$, we get

$$
\sum_{i=1}^{k} \frac{d_{i}}{d_{\mathrm{S}_{m_{1}}}}\left\|I_{i, m_{1}}\right\|_{\infty}=\sum_{i=1}^{k} \frac{\kappa_{m_{1}}}{d_{\mathrm{S}_{m_{1}}}}\left\|\tilde{I}_{i, m_{1}}\right\|_{\infty} \leq k \frac{\kappa_{m_{1}}}{d_{\mathrm{S}_{m_{1}}}}
$$

which contradicts (30) since $\lim _{m \rightarrow \infty} k \frac{\kappa_{m_{1}}}{d_{\mathrm{S}_{m_{1}}}}=k N^{*}<\infty$.

Next, we want to derive a contradiction with (31). By Theorem 2.1, $\mathcal{R}_{0, i}>1$ for every $i \in\{1, \ldots, k\}$. We claim that there exist a subsequent $\left\{S_{m_{2}}\right\}_{m \geq 1}$ of $\left\{S_{m_{1}}\right\}$ and $x_{2} \in \bar{\Omega}$ such that

$$
S_{m_{2}}\left(x_{2}\right) \rightarrow 0 \quad \text { as } \quad m \rightarrow \infty
$$

Indeed, let us first rewrite (33) as

$$
\frac{\kappa_{m_{1}}}{d_{\mathrm{S}_{m_{1}}}}=S_{m_{1}}+\sum_{j=1}^{k} \frac{d_{j}}{d_{\mathrm{S}_{m_{1}}}} I_{j, m_{1}} \quad \forall m \geq 1,
$$

and introduce the variable

$$
\hat{I}_{j, m_{1}}:=\frac{d_{j}}{d_{\mathrm{S}_{m_{1}}}} I_{j, m}=\frac{\kappa_{m_{1}}}{d_{\mathrm{S}_{m_{1}}}} \tilde{I}_{j, m_{1}} \quad m \geq 1,
$$

where $\tilde{I}_{j, m_{1}}$ are introduced above. Since $0<\tilde{I}_{j, m}<1$ for every $m \geq 1$ and $j=$ $1, \ldots, k$, by a priori estimates for elliptic equations, we may suppose that there exist $\left(\tilde{I}_{1, \infty}, \ldots, \tilde{I}_{k, \infty}\right) \in\left[C^{1}(\bar{\Omega})\right]^{k}$ and a subsequence $\left\{m_{2}\right\}_{m \geq 1}$ of $\left\{m_{1}\right\}_{m \geq 1}$ such that

$$
\left(\tilde{I}_{1, m_{2}}, \cdots, \tilde{I}_{k, m_{2}}\right) \rightarrow\left(\tilde{I}_{1, \infty}, \ldots, \tilde{I}_{k, \infty}\right) \text { as } m \rightarrow \infty
$$

in $\left[C^{1}(\bar{\Omega})\right]^{k}$. As a result, by recalling (34), we conclude that $\hat{I}_{j, m_{2}} \rightarrow N^{*} \tilde{I}_{j, \infty}$ as $m \rightarrow \infty$ in $C^{1}(\bar{\Omega})$ for each $j=1, \ldots, k$. This in turn, along with (34) and (41), imply that there is $S_{\infty} \in C^{1}(\bar{\Omega})$ such that $S_{m_{2}} \rightarrow S_{\infty}$ as $m \rightarrow \infty$ in $C^{1}(\bar{\Omega})$. Now, we claim that the set

$$
\left\{x \in \bar{\Omega}: S_{\infty}(x)=0\right\} \neq \emptyset .
$$


If this were false, then by observing that

$$
\begin{cases}0=d_{1} \Delta\left(\frac{I_{1, m_{2}}}{\left\|I_{1, m_{2}}\right\|_{\infty}}\right)-\gamma_{1} \frac{I_{1, m_{2}}}{\left\|I_{1, m_{2}}\right\|_{\infty}}+\frac{\beta_{1} S_{m_{2}}}{S_{m_{2}}+\sum_{j=1}^{k} I_{j, m_{2}}}\left(\frac{I_{1, m_{2}}}{\left\|I_{1, m_{2}}\right\|_{\infty}}\right) & x \in \Omega \\ \partial_{\vec{n}} \frac{I_{1, m_{2}}}{\left\|I_{1, m_{2}}\right\|_{\infty}}=0 & x \in \partial \Omega\end{cases}
$$

for every $m \geq 1$ and $\frac{S_{m_{2}}}{S_{m_{2}}+\sum_{j=1}^{k} I_{j, m_{2}}} \rightarrow \frac{S_{\infty}}{S_{\infty}} \equiv 1$ in $C^{1}(\bar{\Omega})$ as $m \rightarrow \infty$ (note that $\sum_{j=1}^{k} I_{j, m_{2}} \rightarrow 0$ as $m \rightarrow \infty$ in $C^{1}(\bar{\Omega})$ since (39) holds), using a priori estimates for elliptic equations, there is $\varphi_{1} \in C^{2}(\Omega)$ with $\varphi_{1} \geq 0$ and $\left\|\varphi_{1}\right\|_{\infty}=1$, and a subsequence $\left\{I_{1, m_{3}}\right\}_{m \geq 1}$ of $\left\{I_{1, m_{2}}\right\}$ such that $\frac{I_{1, m_{3}}}{\left\|I_{1, m_{3}}\right\|_{\infty}} \rightarrow \varphi_{1}$ as $m \rightarrow \infty$ in $C^{1}(\bar{\Omega})$ and $\varphi_{1}$ is a solution of

$$
0=d_{1} \Delta \varphi_{1}+\left(\beta_{1}-\gamma_{1}\right) \varphi_{1} \quad x \in \Omega, \quad \text { and } \partial_{\vec{n}} \varphi_{1}=0 \quad x \in \partial \Omega .
$$

By the strong maximum principle, we deduce that $\varphi_{1}(x)>0$ for every $x \in \bar{\Omega}$. This implies that we must have $\mathcal{R}_{0,1} \leq 1$, which is a contradiction. Therefore (42) must hold, which yields that (40) also holds. Now, by (40), (41) and (34), we have

$$
\lim _{m \rightarrow \infty} \sum_{i=1}^{k} \frac{d_{i}}{d_{\mathrm{S}_{m}}} I_{i, m}\left(x_{2}\right)=N^{*} \in(0, \infty)
$$

Using the Harnack inequality for elliptic equations, we know that there exists a positive constant $v$ that

$$
\min _{x \in \bar{\Omega}} I_{i, m} \geq v\left\|I_{i, m}\right\|_{\infty} \quad \forall m \geq 1, i=1, \ldots, k
$$

This shows that (43) contradicts (31). The proof is complete.

\section{Appendix B: Proofs of Theorems 2.4 and 2.9}

In this section, we study the existence of EE for (1) and prove Theorems 2.4 and 2.9.

\subsection{B.1 Proof of Theorem 2.9}

We let $k=2$ and set $d_{i}=\varepsilon D_{i}$ for each $i=1,2$, where $D_{1}$ and $D_{2}$ are fixed positive numbers. Let us introduce a few notations. Define

$$
\mathcal{Q}\left(x, I_{1}\right):=\frac{\tilde{\gamma}_{2}(x)\left(1-I_{1}\right)+\tilde{\gamma}_{1}(x) I_{1}}{\tilde{\beta}_{2}(x)\left(1-I_{1}\right)+\tilde{\beta}_{1}(x) I_{1}} \quad \text { for every } 0 \leq I_{1} \leq 1,
$$


where $\left(\tilde{\beta}_{i}, \tilde{\gamma}_{i}\right)=\left(\frac{\beta_{i}}{D_{i}}, \frac{\gamma_{i}}{D_{i}}\right)$ for each $i=1,2$. Next we consider the elliptic equation

$$
\left\{\begin{array}{l}
0=\varepsilon \Delta \tilde{I}_{1}+\left(\tilde{\beta}_{1} \mathcal{Q}\left(x, \tilde{I}_{1}\right)-\tilde{\gamma}_{1}\right) \tilde{I}_{1}, \quad x \in \Omega, \\
\partial_{\vec{n}} \tilde{I}_{1}=0 \quad x \in \partial \Omega, \\
0<\tilde{I}_{1}<1 \quad x \in \Omega .
\end{array}\right.
$$

Observe that $\tilde{\beta}_{1} \mathcal{Q}(\cdot, 1)-\tilde{\gamma}_{1} \equiv 0$. The following result holds.

Lemma 4.2 Suppose that $\mathfrak{R}_{1}-\mathfrak{R}_{2}$ changes sign on $\Omega$. Then there is some $\varepsilon^{*}>0$ such that for every $0<\varepsilon<\varepsilon^{*}$, (45) has at least one positive solution. Furthermore, if $\frac{\beta_{1}}{\beta_{2}}$ is a constant, then (45) has a unique positive solution, which is also linearly stable.

Proof Denote by $\lambda_{0, \varepsilon}$ the principal eigenvalue of (45) linearized at $I_{1} \equiv 0$, that is $\lambda_{0, \varepsilon}=\lambda^{*}\left(\varepsilon, \tilde{\beta}_{1} \mathcal{Q}(\cdot, 0)-\tilde{\gamma}_{1}\right)$. Observe that $\tilde{\beta}_{1} \mathcal{Q}(\cdot, 0)-\tilde{\gamma}_{1}=\tilde{\gamma}_{1}\left(\frac{\Re_{1}}{\mathfrak{R}_{2}}-1\right)$ changes sign on $\Omega$. Since the function $\varepsilon \mapsto \lambda_{0, \varepsilon}$ is strictly decreasing with $\lambda_{0, \varepsilon} \rightarrow$ $-\max _{x \in \bar{\Omega}}\left\{\tilde{\beta}_{1}(x) \mathcal{Q}(x, 0)-\tilde{\gamma}_{1}(x)\right\}<0$ (see Allen et al. 2008), there is $\varepsilon_{1}^{*}>0$ such that $\lambda_{0, \varepsilon}<0$ for every $0<\varepsilon<\varepsilon_{1}^{*}$. For every $0<\varepsilon<\varepsilon_{1}^{*}$, let $\varphi_{0, \varepsilon}^{*}$ be an associated positive eigenfunction of $\lambda_{0, \varepsilon}$ with $\max _{x} \varphi_{0, \varepsilon}^{*}=1$. Next, we denote by $\lambda_{1, \varepsilon}$ the principal eigenvalue of (45) linearized at $I_{1} \equiv 1$, that is $\lambda_{1, \varepsilon}=\lambda^{*}\left(\varepsilon, \tilde{\beta}_{1} \partial_{I} \mathcal{Q}(\cdot, 1)\right)$. Observe that $\tilde{\beta}_{1} \partial_{I_{1}} \mathcal{Q}(\cdot, 1)=\tilde{\gamma}_{2}\left(\frac{\mathfrak{R}_{2}}{\mathfrak{R}_{1}}-1\right)$ changes sign on $\Omega$. Similarly as in the above, there is $\varepsilon_{2}^{*}>0$ such that $\lambda_{1, \varepsilon}<0$ for every $0<\varepsilon<\varepsilon_{2}^{*}$. For every $0<\varepsilon<\varepsilon_{2}^{*}$, let $\varphi_{1, \varepsilon}^{*}$ be an associated eigenfunction of $\lambda_{1, \varepsilon}$ with $\max _{x} \varphi_{1, \varepsilon}^{*}=1$. We set $\varepsilon^{*}=\min \left\{\varepsilon_{1}^{*}, \varepsilon_{2}^{*}\right\}$. Direct computations show that for every $0<\varepsilon<\varepsilon^{*}$, there is $0<\tau_{\varepsilon} \ll 1$ such that

$$
\left\{\begin{array}{l}
0<\varepsilon \Delta\left(\tau_{\varepsilon} \varphi_{0, \varepsilon}^{*}\right)+\left(\tilde{\beta}_{1} \mathcal{Q}\left(\cdot, \tau_{\varepsilon} \varphi_{0, \varepsilon}^{*}\right)-\tilde{\gamma}_{1}\right)\left(\tau_{\varepsilon} \varphi_{0, \varepsilon}^{*}\right) \quad x \in \Omega \\
\partial_{\vec{n}}\left(\tau_{\varepsilon} \varphi_{0, \varepsilon}^{*}\right)=0 \quad x \in \partial \Omega
\end{array}\right.
$$

and

$$
\left\{\begin{array}{l}
0>\varepsilon \Delta\left(1-\tau_{\varepsilon} \varphi_{1, \varepsilon}^{*}\right)+\left(\tilde{\beta}_{1} \mathcal{Q}\left(\cdot, 1-\tau_{\varepsilon} \varphi_{1, \varepsilon}^{*}\right)-\tilde{\gamma}_{1}\right)\left(1-\tau_{\varepsilon} \varphi_{1, \varepsilon}^{*}\right) \quad x \in \Omega \\
\partial_{\vec{n}}\left(1-\tau_{\varepsilon} \varphi_{1, \varepsilon}^{*}\right)=0 \quad x \in \partial \Omega,
\end{array}\right.
$$

that is $\tau_{\varepsilon} \varphi_{0, \varepsilon}^{*}$ and $1-\tau_{\varepsilon} \varphi_{1, \varepsilon}^{*}$ are subsolution and supersolution of (45), respectively. Thus, by the sub-super solution method, there is a solution $\tilde{I}_{1}^{*}(x, \varepsilon)$ of (45) with $\tau_{\varepsilon} \varphi_{0, \varepsilon}^{*}<\tilde{I}_{1}^{*}(\cdot, \varepsilon)<1-\tau_{\varepsilon} \varphi_{1, \varepsilon}^{*}$. This completes the proof of the existence part. Next observe that

$$
\begin{gathered}
\left(\tilde{\beta}_{1} \mathcal{Q}(x, I)-\tilde{\gamma}_{1}\right) I=\tilde{\gamma}_{1}\left(\frac{\Re_{1}}{\mathfrak{R}_{2}}-1\right)\left(1-\frac{I}{I+\frac{d_{1} \beta_{2}}{d_{2} \beta_{1}}(1-I)}\right) I \\
\forall x \in \Omega, I \in[0,1]
\end{gathered}
$$


and that the function

$$
I \mapsto\left(1-\frac{I}{I+\frac{d_{1} \beta_{2}}{d_{2} \beta_{1}}(1-I)}\right) I
$$

is strictly concave down, hence it follows from (Hess 1991, Theorem 0.1, Lemma 0.2), that any non-constant positive solution of (45) is unique and linearly stable if $\frac{\beta_{1}}{\beta_{2}}$ is constant.

Throughout the rest of this subsection, we shall suppose that the assumptions of Lemma 4.2 hold. Let $\varepsilon^{*}$ be given by Lemma 4.2. For every $0<\varepsilon<\varepsilon^{*}$, let $\tilde{I}_{1}^{*}(\cdot, \varepsilon)$ be a solution of (45) given by Lemma 45 and set $\tilde{I}_{2}^{*}(\cdot, \varepsilon)=1-\tilde{I}_{1}^{*}(\cdot, \varepsilon)$. Direct computation shows that

$$
\begin{cases}0=\varepsilon \Delta \tilde{I}_{i}^{*}+\left(\tilde{\beta}_{i} \frac{\sum_{j=1}^{2} \tilde{\gamma}_{j} \tilde{I}_{j}^{*}}{\sum_{j=1}^{2} \tilde{\beta}_{j} \tilde{I}_{j}^{*}}-\tilde{\gamma}_{i}\right) \tilde{I}_{i}^{*} & x \in \Omega, i=1,2, \\ 0=\partial_{\vec{n}} \tilde{I}_{i}^{*} & x \in \partial \Omega, i=1,2, \\ 1=\sum_{j=1}^{2} \tilde{I}_{j}^{*} & \end{cases}
$$

Lemma 4.3 Suppose that $\bar{\Omega}=\{\Re>1\}$ in addition to the hypotheses of Lemma 4.2. Let $\left(\tilde{I}_{1}^{*}(\cdot, \varepsilon), \tilde{I}_{2}^{*}(\cdot, \varepsilon)\right)$ be given above for every $0<\varepsilon<\varepsilon^{*}$. Then there is $0<\tilde{\varepsilon}^{*}<\varepsilon^{*}$ such that for every $0<\varepsilon<\tilde{\varepsilon}^{*}$, the set $\Omega_{\varepsilon}^{*}$ defined by

$$
\Omega_{\varepsilon}^{*}:=\left\{x \in \bar{\Omega}: \sum_{i=1}^{2}\left(\tilde{\beta}_{i}(x)-\tilde{\gamma}_{i}(x)\right) \tilde{I}_{i}^{*}(x, \varepsilon) \leq 0\right\}
$$

is empty. Furthermore, by setting $\tilde{S}^{*}:=\frac{\sum_{i=1}^{2} \tilde{\gamma}_{i} \tilde{I}_{i}^{*} \sum_{j=1}^{2} \frac{\tilde{I}_{j}^{*}}{d_{j}}}{\sum_{i=1}^{2}\left(\tilde{\beta}_{i}-\tilde{\gamma}_{i}\right) \tilde{I}_{i}^{*}}$, it holds that

$$
\begin{cases}0=\varepsilon \Delta \tilde{I}_{i}^{*}+\left(\tilde{\beta}_{i} \frac{\tilde{S}^{*}}{\tilde{S}^{*}+\sum_{j=1}^{2} \frac{\tilde{I}_{j}^{*}}{d_{j}}}-\tilde{\gamma}_{i}\right) \tilde{I}_{i}^{*} \quad x \in \Omega, i=1,2, \\ 0=\partial_{\vec{n}} \tilde{I}_{i}^{*} \\ 1=\sum_{j=1}^{2} \tilde{I}_{j}^{*}\end{cases}
$$

for $0<\varepsilon<\tilde{\varepsilon}^{*}$.

Proof Recall that $\tilde{I}_{i}^{*}(\cdot, \varepsilon)$ satisfies

$$
\begin{cases}0=\varepsilon \Delta \tilde{I}_{i}^{*}+\tilde{\gamma}_{1}\left(\frac{\Re_{1}}{\mathfrak{R}_{2}}-1\right)\left(1-\frac{\tilde{I}_{i}^{*}}{\tilde{I}_{i}^{*}+\frac{d_{1} \beta_{2}}{d_{2} \beta_{1}}\left(1-\tilde{I}_{i}^{*}\right)}\right) \tilde{I}_{i}^{*} & x \in \Omega, \\ 0=\partial_{\vec{n}} \tilde{I}_{i}^{*} & x \in \partial \Omega, \\ 0<\tilde{I}_{i}^{*}<1 . & \end{cases}
$$


Then, it follows from Cantrell and Cosner (2003) that

$$
\lim _{\varepsilon \rightarrow 0} \tilde{I}_{i}^{*}(x, \varepsilon)=0 \quad \text { uniformly on compact subsets of }\left\{\Re_{j}>\Re_{i}\right\}
$$

for every $i \neq j$ in $\{1,2\}$. Since $1=\tilde{I}_{1}^{*}+\tilde{I}_{2}^{*}$, we conclude from (48) that

$$
\begin{aligned}
& \lim _{\varepsilon \rightarrow 0} \tilde{I}_{i}^{*}(x, \varepsilon)=1 \text { uniformly on compact subsets of }\left\{\Re_{i}<\Re_{j}\right\}, \\
& i \neq j, i, j=1,2 .
\end{aligned}
$$

Now, since $\bar{\Omega}=\{\Re>1\}$, then $\left\{\Re_{i} \leq 1\right\} \subset\left\{\Re_{j}>1\right\}$ for every $i \neq j, i, j=1,2$. Hence, there are open sets $O_{i}$ such that $\left\{\Re_{i} \leq 1\right\} \subset O_{i} \subset \bar{O}_{i} \subset\left\{\Re_{j}>1\right\}$ for every $i \neq j, i, j=1,2$. By (48) and (49),

$$
\liminf _{\varepsilon \rightarrow 0} \min _{x \in \bar{O}_{1} \cup \bar{O}_{2}} \sum_{i=1}^{2}\left(\tilde{\beta}_{i}(x)-\tilde{\gamma}_{i}(x)\right) \tilde{I}_{i}^{*}(x, \varepsilon)>0 .
$$

Thus, since $\min _{x \in \bar{\Omega} \backslash\left(O_{1} \cup O_{2}\right)} \min \left\{\tilde{\beta}_{1}-\tilde{\gamma}_{1}, \tilde{\beta}_{2}-\tilde{\gamma}_{2}\right\}>0$, we conclude that the set $\Omega_{\varepsilon}^{*}$ is empty for $0<\varepsilon \ll 1$. (47) can be easily checked by inspection.

Proof of Theorem 2.9 Suppose that $d_{\mathrm{S}}=0$. Observe that $\Sigma_{1}$ and $\Sigma_{2}$ are both nonempty if and only if $\Re_{1}-\mathfrak{R}_{2}$ changes sign on $\Omega$. Observe also that $\mathfrak{R}_{0}>1$ if and only if $\{x \in \bar{\Omega}: \Re>1\}=\bar{\Omega}$. Let $\tilde{\varepsilon}^{*}>0$ be given by Lemma 4.3 . For every $0<\varepsilon<\tilde{\varepsilon}^{*}$, let $\left(\tilde{S}^{*}(\cdot, \varepsilon), \tilde{I}_{1}^{*}(\cdot, \varepsilon), \tilde{I}_{2}^{*}(\cdot, \varepsilon)\right)$ be the unique positive solution of (47) given by Lemma 4.3 and set $\tilde{\kappa}_{\varepsilon}^{*}=\frac{N}{\int_{\Omega}\left(\tilde{S}^{*}(\cdot, \varepsilon)+\sum_{i=1}^{2} \frac{\tilde{I}_{i}^{*}(\cdot, \varepsilon)}{d_{i}}\right)}$. A direct computation shows that

$$
\begin{gathered}
\left(S^{*}(x, \varepsilon), I_{1}^{*}(x, \varepsilon), I_{2}^{*}(x, \varepsilon)\right)=\left(\tilde{\kappa}_{\varepsilon}^{*} \tilde{S}^{*}(x, \varepsilon), \tilde{\kappa}_{\varepsilon}^{*} \tilde{I}_{1}^{*}(x, \varepsilon), \tilde{k}_{\varepsilon}^{*} \tilde{I}_{2}^{*}(x, \varepsilon)\right) \\
x \in \Omega, 0<\varepsilon<\tilde{\varepsilon}^{*}
\end{gathered}
$$

is a coexistence EE solution of (1) when $d_{\mathrm{S}}=0$. Moreover, if $\frac{\beta_{1}}{\beta_{2}}$ is constant, if follows from Lemma 4.2 that (1) with $d_{\mathrm{S}}=0$ has a unique coexistence EE solution.

\subsection{B.2 Proof of Theorem 2.4}

We prove a general result for the multi-strain model and derive Theorem 2.4 as a corollary for $k=2$. Let $k \geq 2$ and suppose that $\beta_{i}, \gamma_{i} \in C^{2}(\bar{\Omega})$ for each $i=1, \ldots, k$. For every $\left(I_{1}, \ldots, I_{k-1}\right) \in[0,1]^{k-1}$ satisfying $\sum_{i=1}^{k-1} I_{i} \leq 1$, define

$$
\mathcal{Q}\left(I_{1}, \ldots, I_{k-1}\right)=\frac{\tilde{\gamma}_{k}\left(1-\sum_{i=1}^{k-1} I_{i}\right)+\sum_{i=1}^{k-1} \tilde{\gamma}_{i} I_{i}}{\tilde{\beta}_{k}\left(1-\sum_{i=1}^{k-1} I_{i}\right)+\sum_{i=1}^{k-1} \tilde{\beta}_{i} I_{i}},
$$


where $\left(\tilde{\beta}_{i}, \tilde{\gamma}_{i}\right)=\left(\frac{\beta_{i}}{D_{i}}, \frac{\gamma_{i}}{D_{i}}\right)$ for each $i=1, \ldots, k$. Note that $(50)$ is consistent with (44) when $k=2$. Let $\varepsilon>0$ be fixed and consider the elliptic system

$$
\left\{\begin{array}{l}
0=\varepsilon \Delta \tilde{I}_{i}+\left(\tilde{\beta}_{i} \mathcal{Q}\left(\tilde{I}_{1}, \ldots, \tilde{I}_{k-1}\right)-\tilde{\gamma}_{i}\right) \tilde{I}_{i}, \quad x \in \Omega, i=1, \ldots, k-1 \\
\partial_{\vec{n}} \tilde{I}_{i}=0 \quad x \in \partial \Omega, i=1, \ldots, k-1 \\
0<\sum_{i=1}^{k-1} \tilde{I}_{i}<1 \quad x \in \Omega
\end{array}\right.
$$

Let $\left(\tilde{I}_{1}^{*}(\cdot, \varepsilon), \ldots, \tilde{I}_{k-1}^{*}(\cdot, \varepsilon)\right)$ be a positive solution of $(51)$ and set $\tilde{I}_{k}^{*}(\cdot, \varepsilon)=1-$ $\sum_{i=1}^{k-1} \tilde{I}_{i}^{*}$. For the moment, we shall suppose that

$$
\sum_{i=1}^{k}\left(\tilde{\beta}_{i}-\tilde{\gamma}_{i}\right) \tilde{I}_{i}^{*}(x, \varepsilon)>0 \text { for } x \in \bar{\Omega}
$$

and then set

$$
\tilde{S}^{*}(\cdot, \varepsilon)=\frac{\sum_{i=1}^{k} \frac{\tilde{I}_{i}^{*}(\cdot, \varepsilon)}{d_{i}} \sum_{j=1}^{k} \tilde{\gamma}_{j} \tilde{I}_{j}^{*}(\cdot, \varepsilon)}{\sum_{i=1}^{k}\left(\tilde{\beta}_{i}-\tilde{\gamma}_{i}\right) \tilde{I}_{i}^{*}(\cdot, \varepsilon)} \quad \text { and } \quad \tilde{\kappa}_{\varepsilon}^{*}=\frac{N}{\int_{\Omega}\left(\tilde{S}^{*}(\cdot, \varepsilon)+\sum_{i=1}^{k} \frac{\tilde{I}_{i}^{*}(\cdot, \varepsilon)}{d_{i}}\right)}
$$

A direct computation shows that

$$
\mathcal{Q}\left(\tilde{I}_{1}^{*}(\cdot, \varepsilon), \ldots, \tilde{I}_{k-1}^{*}(\cdot, \varepsilon)\right)=\frac{\tilde{S}^{*}(\cdot, \varepsilon)}{\tilde{S}^{*}(\cdot, \varepsilon)+\sum_{i=1}^{k} \frac{\tilde{I}_{i}^{*}(\cdot, \varepsilon)}{d_{i}}}
$$

Note that Lemmas 4.2 and 4.3 provide sufficient conditions for the existence of a positive coexistence solution of (51) satisfying (52) when $k=2$ and $0<\varepsilon \ll 1$. Denote by $\mathcal{L}_{\varepsilon}$ the linear operator given by the linearization of $(51)$ at $\left(\tilde{I}_{1}^{*}(\cdot, \varepsilon), \ldots, \tilde{I}_{k-1}^{*}(\cdot, \varepsilon)\right)$, that is $\mathcal{L}_{\varepsilon}:\left[W_{n}^{2, p}(\Omega)\right]^{k-1} \rightarrow\left[L^{p}(\Omega)\right]^{k-1}$ with

$$
\begin{aligned}
& \mathcal{L}_{\varepsilon}\left(I_{1}, \ldots, I_{k-1}\right)= \\
& \left.\qquad \begin{array}{c}
\varepsilon \Delta I_{1}+\left(\tilde{\beta}_{1} \mathcal{Q}^{*}+\tilde{\beta}_{1} \tilde{I}_{1}^{*} \partial_{I_{1}} \mathcal{Q}^{*}-\tilde{\gamma}_{1}\right) I_{1}+\tilde{\beta}_{1} \tilde{I}_{1}^{*} \sum_{j=1, j \neq 1}^{k-1} \partial_{I_{j}} \mathcal{Q}^{*} I_{j} \\
\varepsilon \Delta I_{2}+\left(\tilde{\beta}_{2} \mathcal{Q}^{*}+\tilde{\beta}_{2} \tilde{I}_{2}^{*} \partial_{I_{2}} \mathcal{Q}^{*}-\tilde{\gamma}_{2}\right) I_{2}+\tilde{\beta}_{2} \tilde{I}_{2}^{*} \sum_{j=1, j \neq 2}^{k-1} \partial_{I_{j}} \mathcal{Q}^{*} I_{j} \\
\vdots \\
\varepsilon \Delta I_{k-1}+\left(\tilde{\beta}_{k-1} \mathcal{Q}^{*}+\tilde{\beta}_{k-1} I_{k-1}^{*} \partial_{I_{k-1}} \mathcal{Q}^{*}-\tilde{\gamma}_{k-1}\right) I_{k-1}+\tilde{\beta}_{k-1} \tilde{I}_{k-1}^{*} \sum_{j=1}^{k-2} \partial_{I_{j}} \mathcal{Q}^{*} I_{j}
\end{array}\right)
\end{aligned}
$$

where $W_{n}^{2, p}(\Omega)=\left\{u \in W^{2, p}(\Omega) \quad: \quad \partial_{\vec{n}} u=0\right.$ on $\left.\partial \Omega\right\}, \mathcal{Q}^{*}:=\mathcal{Q}\left(\tilde{I}_{1}^{*}(\cdot, \varepsilon)\right.$, $\left.\ldots, \tilde{I}_{k-1}^{*}(\cdot, \varepsilon)\right), \partial_{I_{j}} \mathcal{Q}^{*}:=\partial_{I_{j}} \mathcal{Q}\left(\tilde{I}_{1}^{*}(\cdot, \varepsilon), \ldots, \tilde{I}_{k-1}^{*}(\cdot, \varepsilon)\right), j=1, \ldots, k-1$ and $p \gg 1$ 
is fixed. When $k=2$, the operator $\mathcal{L}_{\varepsilon}$ reduces to the single equation

$$
\mathcal{L}_{\varepsilon}\left(I_{1}\right)=\varepsilon \Delta I_{1}+\left(\tilde{\beta}_{1} \mathcal{Q}\left(\tilde{I}_{1}^{*}\right)+\tilde{\beta}_{1} \tilde{I}_{1}^{*} \partial_{I_{1}} \mathcal{Q}\left(\tilde{I}_{1}^{*}\right)-\tilde{\gamma}_{1}\right) I_{1} \quad \forall I_{1} \in W_{n}^{2, p}(\Omega)
$$

Next, consider the Banach spaces $\mathcal{X}:=\mathbb{R} \times W_{n}^{2, p}(\Omega) \times\left[W_{n}^{2, p}(\Omega)\right]^{k}$ and $\mathcal{Y}:=$ $\mathbb{R} \times W_{n}^{2, p}(\Omega) \times\left[L^{p}(\Omega)\right]^{k}$ and the open subset $\mathcal{U}:=\left\{\left(\kappa, S, I_{1}, \ldots, I_{k}\right) \in \mathcal{X}: \kappa>\right.$ $\left.0, S>0, I_{i}>0, i=1, \ldots, k\right\}$ of $\mathcal{X}$. For every $\varepsilon>0$, define a mapping $\mathcal{F}_{\varepsilon}$ from $\mathbb{R} \times \mathcal{U} \rightarrow \mathcal{Y}$ by

$$
\mathcal{F}_{\varepsilon}\left(d_{\mathrm{S}}, \kappa, S, I_{1}, \ldots, I_{k}\right)=\left(\begin{array}{l}
N-\kappa \int_{\Omega}\left(S+\sum_{i=1}^{k} \frac{I_{i}}{d_{i}}\right) \\
1-d_{\mathrm{S}} S-\sum_{i=1}^{k} I_{i} \\
\varepsilon \Delta I_{1}+\left(\tilde{\beta}_{1} R\left(S, I_{1}, \ldots, I_{k}\right)-\tilde{\gamma}_{1}\right) I_{1} \\
\varepsilon \Delta I_{2}+\left(\tilde{\beta}_{2} R\left(S, I_{1}, \ldots, I_{k}\right)-\tilde{\gamma}_{2}\right) I_{2} \\
\vdots \\
\varepsilon \Delta I_{k}+\left(\tilde{\beta}_{k} R\left(S, I_{1}, \ldots, I_{k}\right)-\tilde{\gamma}_{k}\right) I_{k}
\end{array}\right),
$$

where $R\left(S, I_{1}, \ldots, I_{k}\right)=\frac{S}{S+\sum_{i=1}^{k} \frac{I_{i}}{d_{i}}}$. For the sake of clarity in the presentation of the arguments, we introduce the notations:

$$
R^{*}=R\left(\tilde{S}^{*}(\cdot, \varepsilon), \tilde{I}_{1}^{*}(\cdot, \varepsilon), \ldots, \tilde{I}_{k}^{*}(\cdot, \varepsilon)\right) \quad \text { and } \quad \partial_{y} R^{*}=\partial_{y} R\left(\tilde{S}^{*}(\cdot, \varepsilon), \tilde{I}_{1}^{*}(\cdot, \varepsilon), \ldots, \tilde{I}_{k}^{*}(\cdot, \varepsilon)\right)
$$

for each $y \in\left\{S, I_{1}, \ldots, I_{k}\right\}$. By (54),

$$
R^{*}=\mathcal{Q}^{*} \quad \text { and } \quad \partial_{I_{j}} \mathcal{Q}^{*}=\frac{\left(\tilde{\gamma}_{i}-\tilde{\gamma}_{k}\right)-\left(\tilde{\beta}_{i}-\tilde{\beta}_{k}\right) R^{*}}{\sum_{i=1}^{k} \tilde{\beta}_{i} \tilde{I}_{i}^{*}(\cdot, \varepsilon)} \quad i=1, \ldots, k-1
$$

One easily checks that

$$
\tilde{E}_{\varepsilon}^{*}:=\left(0, \tilde{\kappa}_{\varepsilon}^{*}, \tilde{S}^{*}(\cdot, \varepsilon), \tilde{I}_{1}^{*}(\cdot, \varepsilon), \ldots, \tilde{I}_{k}^{*}(\cdot, \varepsilon)\right) \in \mathbb{R} \times \mathcal{U} \text { and } \mathcal{F}_{\varepsilon}\left(\tilde{E}_{\varepsilon}^{*}\right)=(0,0,0, \ldots, 0)^{T}
$$


Moreover, the mapping $\mathcal{F}_{\varepsilon}$ is Frechet differentiable and

$$
\begin{aligned}
& D_{\left(\kappa, S, I_{1}, \ldots, I_{k}\right)} \mathcal{F}_{\varepsilon}\left(\tilde{E}_{\varepsilon}^{*}\right)\left(\kappa, S, I_{1}, \ldots, I_{k}\right)= \\
& -\kappa \int_{\Omega}\left(\tilde{S}^{*}+\sum_{i=1}^{k} \frac{\tilde{I}_{i}^{*}}{d_{i}}\right)-\tilde{\kappa}_{\epsilon}^{*} \int_{\Omega}\left(S+\sum_{i=1}^{k} \frac{I_{i}}{d_{i}}\right) \\
& -\sum_{i=1}^{k} I_{i} \\
& \left(\begin{array}{c}
\left.\tilde{\beta}_{1} R^{*}+\tilde{\beta}_{1} \tilde{I}_{1}^{*} \partial_{I_{1}} R^{*}-\tilde{\gamma}_{1}\right) I_{1}+\tilde{\beta}_{1} \tilde{I}_{1}^{*} \sum_{j=1, j \neq 1}^{k} \partial_{I_{j}} R^{*} I_{j}+\tilde{\beta}_{1} \tilde{I}_{1}^{*} \partial_{\mathrm{SR}}^{*} S \\
\vdots \\
\varepsilon \Delta I_{1}+\tilde{\beta}_{k} \tilde{I}_{k}^{*} \sum_{j=1, j \neq k}^{k} \partial_{I_{j}} R^{*} I_{j}+\tilde{\beta}_{k} \tilde{I}_{k}^{*} \partial_{\mathrm{SR}}^{*} S
\end{array}\right)
\end{aligned}
$$

The next lemma shows that if the linear operator $\mathcal{L}_{\varepsilon}$ is invertible, so is the linear operator $D_{\left(\kappa, S, I_{1}, \ldots, I_{k}\right)} \mathcal{F}_{\varepsilon}\left(\tilde{E}_{\varepsilon}^{*}\right)$.

Lemma 4.4 If the linear operator $\mathcal{L}_{\varepsilon}:\left[W_{n}^{2, p}(\Omega)\right]^{k-1} \rightarrow\left[L^{p}(\Omega)\right]^{k-1}$ is invertible, so is the linear operator $D_{\left(\kappa, S, I_{1}, \ldots, I_{k}\right)} \mathcal{F}_{\varepsilon}\left(\tilde{E}_{\varepsilon}^{*}\right): \mathcal{X} \rightarrow \mathcal{Y}$.

Proof Suppose that the linear operator $\mathcal{L}_{\varepsilon}$ is invertible. The proof is in two steps. In the first step, we show that $D_{\left(\kappa, S, I_{1}, \ldots, I_{k}\right)} \mathcal{F}_{\varepsilon}\left(\tilde{E}_{\varepsilon}^{*}\right)$ is one-to-one. In the second step, we show that it is onto.

Step 1 The linear operator $D_{\left(\kappa, S, I_{1}, \ldots, I_{k}\right)} \mathcal{F}_{\varepsilon}\left(\tilde{E}_{\varepsilon}^{*}\right)$ is one-to-one. Indeed, let $\left(\kappa, S, I_{1}, \ldots, I_{k}\right) \in \mathcal{N}\left(D_{\left(\kappa, S, I_{1}, \ldots, I_{k}\right)} \mathcal{F}_{\varepsilon}\left(\tilde{E}_{\varepsilon}^{*}\right)\right)$, that is

$$
D_{\left(\kappa, S, I_{1}, \ldots, I_{k}\right)} \mathcal{F}_{\varepsilon}\left(\tilde{E}_{\varepsilon}^{*}\right)\left(\kappa, S, I_{1}, \ldots, I_{k}\right)=(0,0,0, \ldots, 0)^{T} .
$$

From the second equation in (57), we get $I_{k}=-\sum_{i=1}^{k-1} I_{i}$. For each $i \in\{1, \ldots, k-1\}$, inserting $I_{k}=-\sum_{j=1}^{k-1} I_{j}$ in the equation satisfied by $I_{i}$ in (57) gives

$$
\begin{aligned}
0= & \varepsilon \Delta I_{i}+\left[\tilde{\beta}_{i} R^{*}+\tilde{\beta}_{i} \tilde{I}_{i}^{*}\left(\partial_{I_{i}} R^{*}-\partial_{I_{k}} R^{*}\right)-\tilde{\gamma}_{i}\right] I_{i} \\
& +\tilde{\beta}_{i} \tilde{I}_{i}^{*} \sum_{j=1, j \neq i}^{k-1}\left[\partial_{I_{j}} R^{*}-\partial_{I_{k}} R^{*}\right] I_{j}+\tilde{\beta}_{i} \tilde{I}_{i}^{*} S \partial_{\mathrm{S}} R^{*} .
\end{aligned}
$$

Inserting $I_{k}=-\sum_{i=1}^{k-1} I_{i}$ in the last equation of (57) gives

$$
0=-\varepsilon \Delta \sum_{i=1}^{k-1} I_{i}-\left[\tilde{\beta}_{k} R^{*}+\tilde{\beta}_{k} \tilde{I}_{k}^{*} \partial_{I_{k}} R^{*}-\tilde{\gamma}_{k}\right] \sum_{i=1}^{k-1} I_{i}+\tilde{\beta}_{k} \tilde{I}_{k}^{*} \sum_{i=1}^{k-1} \partial_{I_{i}} R^{*} I_{i}+\tilde{\beta}_{k} \tilde{I}_{k}^{*} S \partial_{\mathrm{SR}}^{*}
$$

Summing up Eqs. (58) and (59) side-by-side gives

$$
0=\sum_{i=1}^{k-1} L_{i} I_{i}+S \partial_{\mathrm{SR}}^{*} \sum_{i=1}^{k} \tilde{\beta}_{i} \tilde{I}_{i}^{*}
$$


where

$$
\begin{aligned}
L_{i}= & \left(\tilde{\beta}_{i}-\tilde{\beta}_{k}\right) R^{*}-\left(\tilde{\gamma}_{i}-\tilde{\gamma}_{k}\right)+\left[\tilde{\beta}_{i} \tilde{I}_{i}^{*}+\tilde{\beta}_{k} \tilde{I}_{k}^{*}\right] \partial_{I_{i}} R^{*} \\
& -\left[\tilde{\beta}_{i} \tilde{I}_{i}^{*}+\tilde{\beta}_{k} \tilde{I}_{k}^{*}\right] \partial_{I_{k}} R^{*}+\sum_{j=1, j \neq i}^{k-1} \tilde{\beta}_{j} \tilde{I}_{j}^{*}\left(\partial_{I_{i}} R^{*}-\partial_{I_{i}} R^{*}\right) \\
= & \left(\tilde{\beta}_{i}-\tilde{\beta}_{k}\right) R^{*}-\left(\tilde{\gamma}_{i}-\tilde{\gamma}_{k}\right)+\left[\partial_{I_{i}} R^{*}-\partial_{I_{k}} R^{*}\right] \sum_{j=1}^{k} \tilde{\beta}_{j} \tilde{I}_{j}^{*} .
\end{aligned}
$$

Solving for $S \partial_{\mathrm{S}} R^{*}$ in (60), we get

$$
S \partial_{\mathrm{S}} R^{*}=-\sum_{i=1}^{k-1} \frac{L_{i} I_{i}}{\sum_{j=1}^{k} \tilde{\beta}_{j} \tilde{I}_{j}^{*}} .
$$

Finally combining (62) and (58), we obtain

$$
\begin{aligned}
0= & \varepsilon \Delta I_{i}+\left[\tilde{\beta}_{i} R^{*}+\tilde{\beta}_{i} \tilde{I}_{i}^{*}\left(\partial_{I_{i}} R^{*}-\partial_{I_{k}} R^{*}-\frac{L_{i}}{\sum_{j=1}^{k} \tilde{\beta}_{j} \tilde{I}_{j}^{*}}\right)-\tilde{\gamma}_{i}\right] I_{i} \\
& +\tilde{\beta}_{i} \tilde{I}_{i}^{*} \sum_{j=1, j \neq i}^{k-1}\left[\partial_{I_{j}} R^{*}-\partial_{I_{k}} R^{*}-\frac{L_{j}}{\sum_{j=1}^{k} \tilde{\beta}_{j} \tilde{I}_{j}^{*}}\right] I_{j}
\end{aligned}
$$

for each $i=1, \ldots, k-1$. But, by (56) and (61), we have

$$
\partial_{I_{j}} R^{*}-\partial_{I_{k}} R^{*}-\frac{L_{j}}{\sum_{j=1}^{k} \tilde{\beta}_{j} \tilde{I}_{j}^{*}}=\partial_{I_{j}} \mathcal{Q}^{*}
$$

for every $j=1, \ldots, k-1$. As a result, it follows from (63) and the definition of the operator $\mathcal{L}_{\varepsilon}$ that $\mathcal{L}_{\varepsilon}\left(I_{1}, \ldots, I_{k-1}\right)=0$, which implies that $\left(I_{1}, \ldots, I_{k-1}\right)=0$, since the operator $\mathcal{L}_{\varepsilon}$ is invertible. Recalling that $I_{k}=-\sum_{i=1}^{k-1} I_{i}$ and Eq. (62), we also get that $I_{k}=0$ and $S=0$. It then follows from the first equation in (57) that $\kappa=0$. This completes the proof of step 1.

Step 2 The linear operator $D_{\left(\kappa, S, I_{1}, \ldots, I_{k}\right)} \mathcal{F}_{\varepsilon}\left(\tilde{E}_{\varepsilon}^{*}\right)$ is onto. Indeed, let $Y=\left(\hat{\kappa}, \hat{S}, \hat{I}_{1}, \ldots, \hat{I}_{k}\right) \in \mathcal{Y}$ and we show that there is $X=\left(\kappa, S, I_{1}, \ldots, I_{k}\right) \in \mathcal{X}$ satisfying 
$D_{\left(\kappa, S, I_{1}, \ldots, I_{k}\right)} \mathcal{F}_{\varepsilon}\left(\tilde{E}_{\varepsilon}^{*}\right)(X)=Y$, that is

$$
\left\{\begin{array}{l}
\hat{\kappa}=-\kappa \int_{\Omega}\left(\tilde{S}^{*}+\sum_{i=1}^{k} \frac{\tilde{I}_{i}^{*}}{d_{i}}\right)-\tilde{\kappa}^{*} \int_{\Omega}\left(S+\sum_{i=1}^{k} \frac{I_{i}}{d_{i}}\right) \\
\hat{S}=-\sum_{i=1}^{k} I_{i} \\
\hat{I}_{1}=\varepsilon \Delta I_{1}+\left(\tilde{\beta}_{1} R^{*}+\tilde{\beta}_{1} \tilde{I}_{1}^{*} \partial_{I_{1}} R^{*}-\tilde{\gamma}_{1}\right) I_{1}+\tilde{\beta}_{1} \tilde{I}_{1}^{*} \sum_{j=1, j \neq 1}^{k} \partial_{I_{j}} R^{*} I_{j}+\tilde{\beta}_{1} \tilde{I}_{1}^{*} \partial_{\mathrm{SR}}^{*} S \\
\hat{I}_{2}=\varepsilon \Delta I_{2}+\left(\tilde{\beta}_{2} R^{*}+\tilde{\beta}_{2} \tilde{I}_{2}^{*} \partial_{I_{2}} R^{*}-\tilde{\gamma}_{2}\right) I_{2}+\tilde{\beta}_{2} \tilde{I}_{2}^{*} \sum_{j=1, j \neq 2}^{k} \partial_{I_{j}} R^{*} I_{j}+\tilde{\beta}_{2} \tilde{I}_{2}^{*} \partial_{\mathrm{SR}}^{*} S \\
\quad \vdots \\
\hat{I}_{k}=\varepsilon \Delta I_{k}+\left(\tilde{\beta}_{k} R^{*}+\tilde{\beta}_{k} \tilde{I}_{k}^{*} \partial_{I_{k}} R^{*}-\tilde{\gamma}_{k}\right) I_{k}+\tilde{\beta}_{k} \tilde{I}_{k}^{*} \sum_{j=1, j \neq k}^{k} \partial_{I_{j}} R^{*} I_{j}+\tilde{\beta}_{k} \tilde{I}_{k}^{*} \partial_{\mathrm{SR}}^{*} S .
\end{array}\right.
$$

Solving for $I_{k}$ in the second equation of (64) yields $I_{k}=-\left(\hat{S}+\sum_{i=1}^{k-1} I_{i}\right)$. Inserting this in the last $k$-equations of (64), for each $i=1 \cdots, k-1$, we get

$$
\begin{array}{r}
\hat{I}_{i}+\tilde{\beta}_{i} \tilde{I}_{i}^{*} \hat{S} \partial_{I_{k}} R^{*}=\varepsilon \Delta I_{i}+\left(\tilde{\beta}_{i} R^{*}+\tilde{\beta}_{i} \tilde{I}_{i}^{*}\left(\partial_{I_{i}} R^{*}-\partial_{I_{k}} R^{*}\right)-\tilde{\gamma}_{i}\right) I_{i} \\
+\tilde{\beta}_{i} \tilde{I}_{i}^{*} \sum_{j=1, j \neq i}^{k-1}\left(\partial_{I_{j}} R^{*}-\partial_{I_{k}} R^{*}\right) I_{j}+\tilde{\beta}_{i} \tilde{I}_{i}^{*} \partial_{\mathrm{SR}}^{*} S
\end{array}
$$

and the last equation in (64) becomes

$$
\begin{aligned}
\hat{I}_{k}+ & \varepsilon \Delta \hat{S}+\left(\tilde{\beta}_{k} R^{*}+\tilde{\beta}_{k} \tilde{I}_{k}^{*} \partial_{I_{k}} R^{*}-\tilde{\gamma}_{k}\right) \hat{S} \\
= & -\varepsilon \Delta \sum_{i=1}^{k-1} I_{i}-\left[\tilde{\beta}_{k} R^{*}+\tilde{\beta}_{k} \tilde{I}_{k}^{*} \partial_{I_{k}} R^{*}-\tilde{\gamma}_{k}\right] \sum_{i=1}^{k-1} I_{i} \\
& +\tilde{\beta}_{k} \tilde{I}_{k}^{*} \sum_{i=1}^{k-1} \partial_{I_{i}} R^{*} I_{i}+\tilde{\beta}_{k} \tilde{I}_{k}^{*} S \partial_{\mathrm{SR}}^{*}
\end{aligned}
$$

Note the similarity between Eqs. (58) and (65) and between Eqs. (59) and (66). Hence, recalling the expression of $L_{i}, i=1, \ldots, k-1$, from (65), by adding up the $k-1$ equations in (65) with Eq. (66), we obtain

$$
\begin{aligned}
& \sum_{i=1}^{k} \hat{I}_{i}+\varepsilon \Delta \hat{S}+\left(\tilde{\beta}_{k} R^{*}+\partial_{I_{k}} R^{*} \sum_{i=1}^{k} \tilde{\beta}_{i} \tilde{I}_{i}^{*}-\tilde{\gamma}_{k}\right) \hat{S} \\
& \quad=\sum_{i=1}^{k-1} L_{i} I_{i}+S \partial_{\mathrm{S}} R^{*} \sum_{i=1}^{k} \tilde{\beta}_{k} \tilde{I}_{k}^{*} .
\end{aligned}
$$

Setting

$$
\hat{Z}:=\frac{\sum_{i=1}^{k} \hat{I}_{i}+\varepsilon \Delta \hat{S}+\left(\tilde{\beta}_{k} R^{*}+\partial_{I_{k}} R^{*} \sum_{i=1}^{k} \tilde{\beta}_{i} \tilde{I}_{i}^{*}-\tilde{\gamma}_{k}\right) \hat{S}}{\sum_{j=1}^{k} \tilde{\beta}_{j} \tilde{I}_{j}^{*}}
$$


and solving for $S \partial_{\mathrm{SR}}^{*}$ in (67) yield

$$
S \partial_{\mathrm{SR}}^{*}=\hat{Z}-\sum_{i=1}^{k-1} \frac{L_{i}}{\sum_{j=1}^{k} \tilde{\beta}_{j} \tilde{I}_{j}^{*}} I_{i} .
$$

Hence, inserting this expression in (65) and recalling (56), we obtain

$$
\left(\hat{Z}_{1}, \ldots, \hat{Z}_{k-1}\right)=\mathcal{L}_{\varepsilon}\left(I_{1}, \ldots, I_{k-1}\right)
$$

where $\hat{Z}_{i}=\hat{I}_{i}+\tilde{\beta}_{i} \tilde{I}_{i}^{*}\left(\hat{S} \partial_{I_{k}} R^{*}-\hat{Z}\right) \in L^{p}(\Omega)$ for each $i=1, \ldots, k-1$. Since the operator $\mathcal{L}_{\varepsilon}$ is invertible, there is a unique $\left(I_{1}, \ldots, I_{k-1}\right) \in\left[W_{n}^{2, p}(\Omega)\right]^{k-1}$ solving (69). Note also that $I_{k}$ is uniquely determined by $I_{k}=-\left(\hat{S}+\sum_{i=1}^{k-1} I_{i}\right)$ and that $S$ is uniquely determined by Eq. (68) since

$$
\partial_{\mathrm{S}} R^{*}=\frac{\sum_{i=1}^{k} \frac{\tilde{I}_{i}^{*}(\cdot, \varepsilon)}{d_{i}}}{\tilde{S}^{*}(\cdot, \varepsilon)+\sum_{i=1}^{k} \frac{\tilde{I}_{i}^{*}(\cdot, \varepsilon)}{d_{i}}}>0 .
$$

Now, with $\left(S, I_{1}, \ldots, I_{k}\right)$ obtained, we can solve for $\kappa$ from the first equation in (64). This completes the proof.

Now, we can state our main result on the existence of coexistence endemic equilibrium for $d_{\mathrm{S}}>0$.

Theorem 4.5 Let $k \geq 2, \varepsilon>0$, and $d_{i}=\varepsilon_{i}$, $i=1, \ldots, k$. Let $\left(\tilde{I}_{1}^{*}(\cdot, \varepsilon), \ldots, \tilde{I}_{k-1}^{*}(\cdot, \varepsilon)\right)$ be a positive coexistence solution of $(51)$ satisfying (52) and define $\left(\tilde{S}^{*}(\cdot, \varepsilon), \tilde{\kappa}_{\varepsilon}^{*}\right)$ by (53). Suppose that the linearized operator $\mathcal{L}_{\varepsilon}$ of $(51)$ at $\left(\tilde{I}_{1}^{*}(\cdot, \varepsilon), \ldots, \tilde{I}_{k-1}^{*}(\cdot, \varepsilon)\right)$ is invertible. Then there is $0<d_{\mathrm{S}, \varepsilon} \ll 1$ such that for every $0<d_{\mathrm{S}}<d_{S, \varepsilon}$, (1) has a coexistence steady-state solution $\left(S^{*}\left(\cdot ; d_{\mathrm{S}}, \varepsilon\right), I_{1}^{*}\left(\cdot, d_{\mathrm{S}}, \varepsilon\right), \ldots, I_{k}^{*}\left(\cdot, d_{\mathrm{S}}, \varepsilon\right)\right)$. Moreover, for every $d_{\mathrm{S}} \in\left(0, d_{S, \varepsilon}\right)$, $\left(S^{*}\left(\cdot ; d_{\mathrm{S}}, \varepsilon\right), I_{1}^{*}\left(\cdot, d_{\mathrm{S}}, \varepsilon\right), \ldots, I_{k}^{*}\left(\cdot, d_{\mathrm{S}}, \varepsilon\right)\right)$ is an isolated coexistent steady-state solution and

$\lim _{d_{\mathrm{S}} \rightarrow 0}\left(S^{*}\left(\cdot ; d_{\mathrm{S}}, \varepsilon\right), I_{1}^{*}\left(\cdot, d_{\mathrm{S}}, \varepsilon\right), \ldots, I_{k}^{*}\left(\cdot, d_{\mathrm{S}}, \varepsilon\right)\right)=\left(S^{*}(\cdot ; \varepsilon), I_{1}^{*}(\cdot, \varepsilon), \ldots, I_{k}^{*}(\cdot, \varepsilon)\right)$,

where $\left(S^{*}(\cdot ; \varepsilon), I_{1}^{*}(\cdot, \varepsilon), \ldots, I_{k}^{*}(\cdot, \varepsilon)\right)$ is also an isolated coexistent steady-state solution of (1) for $d_{\mathrm{S}}=0$.

Proof By Lemma 4.4 and the implicit function theorem, there exist $0<d_{\varepsilon}^{*} \ll 1$, an open subset $\mathcal{U}_{\varepsilon}^{*}$ of $\left(\tilde{\kappa}_{\varepsilon}^{*}, \tilde{S}^{*}(\cdot, \varepsilon), \tilde{I}_{1}^{*}(\cdot, \varepsilon), \ldots, \tilde{I}_{k}^{*}(\cdot, \varepsilon)\right)$ in $\mathcal{U}$ and a $C^{1}$ function

$$
\left(-d_{\varepsilon}^{*}, d_{\varepsilon}^{*}\right) \ni d_{\mathrm{S}} \mapsto\left(\tilde{\kappa}_{\varepsilon}^{*}\left(d_{\mathrm{S}}\right), \tilde{S}^{*}\left(\cdot ; d_{\mathrm{S}}, \varepsilon\right), \tilde{I}_{1}^{*}\left(\cdot ; d_{\mathrm{S}}, \varepsilon\right), \ldots, \tilde{I}_{k}^{*}\left(\cdot ; d_{\mathrm{S}}, \varepsilon\right)\right) \in \mathcal{U}_{\varepsilon}^{*}
$$


such that for each $0<d_{\mathrm{S}}<d_{\varepsilon}^{*},\left(d_{\mathrm{S}}, \tilde{\kappa}_{\varepsilon}^{*}\left(d_{\mathrm{S}}\right), \tilde{S}^{*}\left(\cdot ; d_{\mathrm{S}}, \varepsilon\right), \tilde{I}_{1}^{*}\left(\cdot ; d_{\mathrm{S}}, \varepsilon\right), \ldots, \tilde{I}_{k}^{*}\left(\cdot ; d_{\mathrm{S}}, \varepsilon\right)\right)$ is the unique solution of the equation $\mathcal{F}_{\varepsilon}\left(d_{\mathrm{S}}, \kappa, S, I_{1}, \ldots, I_{k}\right)=0$ in $\left(-d_{\varepsilon}^{*}, d_{\varepsilon}^{*}\right) \times \mathcal{U}_{\varepsilon}^{*}$. By direct computations, for every $0<d_{\mathrm{S}}<d_{\varepsilon}^{*}$, by defining

$$
S^{*}\left(\cdot ; d_{\mathrm{S}}, \varepsilon\right)=\tilde{\kappa}_{\varepsilon}^{*}\left(d_{\mathrm{S}}\right) \tilde{S}^{*}\left(\cdot ; d_{\mathrm{S}}, \varepsilon\right) \quad \text { and } \quad I_{i}^{*}\left(\cdot ; d_{\mathrm{S}}, \varepsilon\right)=\frac{\tilde{\kappa}_{\varepsilon}^{*}\left(d_{\mathrm{S}}\right)}{d_{i}} \tilde{I}_{i}^{*}\left(\cdot ; d_{\mathrm{S}}, \varepsilon\right), i=1, \ldots, k \text {, }
$$

then $\left(S^{*}\left(\cdot ; d_{\mathrm{S}}, \varepsilon\right), I_{1}^{*}\left(\cdot ; d_{\mathrm{S}}, \varepsilon\right), \ldots, I_{k}^{*}\left(\cdot ; d_{\mathrm{S}}, \varepsilon\right)\right)$ satisfies the assertion of the theorem.

As a corollary of Theorem 4.5, we deduce Theorem 2.4.

Proof of Theorem 2.4 Suppose that $k=2$. By Lemmas 4.2 and 4.3, we know that there is $\tilde{\varepsilon}^{*}>0$ such that for $0<\varepsilon<\tilde{\varepsilon}^{*}$, (45) (equivalently (51)) has a solution satisfying $(52)$, with $\tilde{I}_{1}^{*}\left(\cdot ; d_{\mathrm{S}}, \varepsilon\right)$ being linearly stable. Hence, the result follows from Theorem 4.5 .

\section{Appendix C: Proofs of Theorems 2.5, 2.6 and 2.8}

In the current section, we fix $d_{\mathrm{S}}=0$ and present the proofs of Theorems 2.5, 2.6 and 2.8. First, we study the stability of the disease-free equilibrium and prove Theorem 2.6.

Lemma 5.6 Suppose $k \geq 1, d_{\mathrm{S}}=0,\left|\cap_{i=1}^{k} \Omega \backslash H_{i}^{+}\right|>0$ and let $\left(S(t, x), I_{1}(t, x), \ldots, I_{k}(t, x)\right)$ be a positive classical solution of (1). Then $\left\|\sum_{i=1}^{k} I_{i}(t, \cdot)\right\|_{\infty} \rightarrow 0$ as $t \rightarrow \infty$. Furthermore, if $\Re_{0}<1$, there is $S^{*} \in C(\bar{\Omega})$ such that $S(t, \cdot) \rightarrow S^{*}(\cdot)$ as $t \rightarrow \infty$ uniformly in $x \in \Omega$.

Proof The proof is divided into two steps.

Step 1 In this step, we shall show that $\left\|\sum_{i=1}^{k} I_{i}(t, \cdot)\right\|_{\infty} \rightarrow 0$ as $t \rightarrow \infty$ and that there exists $S_{-}^{*}: \cap_{i=1}^{k}\left(\Omega \backslash H_{i}^{+}\right) \rightarrow(0, \infty)$ such that $\left\|S(t, \cdot)-S_{-}^{*}\right\|_{L^{1}\left(\cap_{i=1}^{k}\left(\Omega \backslash H_{i}^{+}\right)\right)} \rightarrow 0$ as $t \rightarrow \infty$. Indeed, observe that any solution $\left(S(t, x), I_{1}(t, x), \ldots, I_{k}(t, x)\right)$ of (1) satisfies

$$
\begin{aligned}
\partial_{t} S(t, x) & =\frac{\sum_{i=1}^{k}\left(\gamma_{i}-\beta_{i}\right) I_{i} S+\sum_{i=1}^{k} \gamma_{i} I_{i} \sum_{j=1}^{k} I_{j}}{S+\sum_{j=1}^{k} I_{j}} \\
& \geq \frac{\underline{\gamma}\left(\min _{y \in \bar{\Omega}} \sum_{i=1}^{k} I_{i}(t, y)\right)^{2}}{S(t, x)+\min _{y \in \bar{\Omega}} \sum_{i=1} I_{i}(t, y)} \forall t>0,
\end{aligned}
$$

for every $x \in \cap_{i=1}^{k}\left(\Omega \backslash H_{i}^{+}\right)$, where $\underline{\gamma}=\min _{x \in \bar{\Omega}} \min \left\{\gamma_{i}(x): i=1 \cdots, k\right\}$. Note that we have used the fact that the function $(0, \infty) \ni \tau \mapsto \frac{\tau^{2}}{a+\tau}$ is increasing for every $a \geq 0$. Hence, the function $t \mapsto S(t, x)$ is strictly increasing for every $x \in \cap_{i=1}^{k}\left(\Omega \backslash H_{i}^{+}\right)$. Thus, $S(t, \cdot)$ converges to some function $S_{-}^{*}(\cdot)$ on $\cap_{i=1}^{k}\left(\Omega \backslash H_{i}^{+}\right)$ 
as $t \rightarrow \infty$. Since $S(t, x) \geq 0$ for every $x \in \Omega$, the Lebesgue monotone convergence theorem implies that

$$
\lim _{t \rightarrow \infty} \int_{\cap_{i=1}^{k}\left(\Omega \backslash H_{i}^{+}\right)} S(t, x)=\int_{\cap_{i=1}^{k}\left(\Omega \backslash H_{i}^{+}\right)} S_{-}^{*}(x) \mathrm{d} x .
$$

But, by (13),

$$
N=\int_{\Omega}\left(S(t, x)+\sum_{i=1}^{k} I_{i}(t, x)\right) \mathrm{d} x \geq \int_{\cap_{i=1}^{k}\left(\Omega \backslash H_{i}^{+}\right)} S(t, x) \mathrm{d} x \quad \forall t \geq 0 .
$$

Whence, by (71), $S_{-}^{*} \in L^{1}\left(\cap_{i=1}^{k}\left(\Omega \backslash H_{i}^{+}\right)\right)$and

$$
\lim _{t \rightarrow \infty}\left\|S(t, \cdot)-S_{-}^{*}\right\|_{L^{1}\left(\cap_{i=1}^{k}\left(\Omega \backslash H_{i}^{+}\right)\right)}=0 .
$$

Next, recalling that $\left|\cap_{i=1}^{k}\left(\Omega \backslash H_{i}^{+}\right)\right|>0$ and $\left\|S_{-}^{*}\right\|_{L^{1}\left(\cap_{i=1}^{k}\left(\Omega \backslash H_{i}^{+}\right)\right)} \leq N<\infty$, we deduce that $S_{-}^{*}$ is finite almost everywhere on $\cap_{i=1}^{k}\left(\Omega \backslash H_{i}^{+}\right)$. So we can choose $x_{0} \in \cap_{i=1}^{k}\left(\Omega \backslash H_{i}^{+}\right)$such that $S_{-}^{*}\left(x_{0}\right) \in(0, \infty)$. This together with inequalities (70), (14) and (16) give that

$$
\begin{aligned}
S_{-}^{*}\left(x_{0}\right) & \geq S\left(0, x_{0}\right)+\underline{\gamma} \int_{0}^{\infty} \frac{\left[\min _{y \in \bar{\Omega}} \sum_{i=1}^{k} I_{i}(t, y)\right]^{2}}{S_{-}^{*}\left(x_{0}\right)+\min _{y \in \bar{\Omega}} \sum_{i=1}^{k} I_{i}(t, y)} \mathrm{d} t \\
& \geq S\left(0, x_{0}\right)+\frac{\underline{\gamma}}{\tilde{c}_{1}\left(S\left(x_{0}\right)+c_{1} N\right)} \int_{1}^{\infty}\left\|\sum_{i=1}^{k} I_{i}(t)\right\|_{\infty}^{2} \mathrm{~d} t .
\end{aligned}
$$

Therefore, since the map $[1, \infty) \mapsto\left\|\sum_{i=1}^{k} I_{i}(t)\right\|_{\infty}$ is uniformly continuous, it follows from (15) that

$$
\lim _{t \rightarrow \infty}\left\|\sum_{i=1}^{k} I_{i}(t, \cdot)\right\|_{\infty}^{2}=0
$$

which yields that $\lim _{t \rightarrow \infty}\left\|\sum_{i=1}^{k} I_{i}(t, \cdot)\right\|_{\infty}=0$. It is also clear from (70) that $S_{-}^{*}(x)>S(0, x) \geq 0$ for every $x \in \cap_{i=1}^{k}\left(\Omega \backslash H_{i}^{+}\right)$.

Step 2 In this step, we suppose that $\mathfrak{R}_{0}<1$ and then show that

$$
\int_{0}^{\infty}\left\|S_{t}(t, \cdot)\right\|_{\infty} \mathrm{d} t<\infty
$$

Since $\Re_{0}<1$, then $H^{-}$has positive measure. So the point $x_{0}$ above can be chosen such that $x_{0} \in H^{-}$, since $H^{-} \subset \cap_{i=1}^{k}\left(\Omega \backslash H_{i}^{+}\right)$and $S_{-}^{*}$ is finite almost everywhere 
on $\cap_{i=1}^{k}\left(\Omega \backslash H_{i}^{+}\right)$. Observe that

$$
\begin{aligned}
S_{t}\left(\cdot, x_{0}\right) & =\sum_{i=1}^{k}\left(\left(\gamma_{i}\left(x_{0}\right)-\beta_{i}\left(x_{0}\right)\right)+\frac{\beta_{i}\left(x_{0}\right) \sum_{j=1}^{k} I_{j}\left(t, x_{0}\right)}{S\left(t, x_{0}\right)+\sum_{j=1}^{k} I_{j}\left(t, x_{0}\right)}\right) I_{i}\left(t, x_{0}\right) \\
& >\min _{i=1, \ldots, k}\left(\gamma_{i}\left(x_{0}\right)-\beta_{i}\left(x_{0}\right)\right) \sum_{i=1}^{k} I_{i}\left(t, x_{0}\right) \quad t>0 .
\end{aligned}
$$

Hence, by (16), there is a positive constant $C>0$ such that

$$
S_{-}^{*}\left(x_{0}\right) \geq S\left(0, x_{0}\right)+C \min _{1 \leq i \leq k}\left(\gamma_{i}\left(x_{0}\right)-\beta_{i}\left(x_{0}\right)\right) \int_{0}^{\infty}\left\|\sum_{i=1}^{k} I(t, \cdot)\right\|_{\infty} \mathrm{d} t,
$$

which implies that $\int_{0}^{\infty}\left\|\sum_{i=1}^{k} I_{i}(t, \cdot)\right\|_{\infty}<\infty$. As a result, we conclude that

$$
\int_{0}^{\infty}\left\|S_{t}(t, \cdot)\right\|_{\infty} \mathrm{d} t \leq \sum_{i=1}^{k}\left\|2 \beta_{i}+\gamma_{i}\right\|_{\infty} \int_{0}^{\infty}\left\|\sum_{i=1}^{k} I_{i}(t, \cdot)\right\|_{\infty}<\infty .
$$

Whence

$$
\lim _{t \rightarrow \infty}\left\|S(t, \cdot)-S_{0}(\cdot)-\int_{0}^{\infty} S_{t}(s, \cdot) \mathrm{d} s\right\|_{\infty}=0
$$

that is $S(t, \cdot) \rightarrow S^{*}(x):=S_{0}(x)+\int_{0}^{\infty} S_{t}(s, x) \mathrm{d} s \in C(\bar{\Omega})$ as $t \rightarrow \infty$ uniformly on $\bar{\Omega}$.

Next, for each $i=1, \ldots, k$, let $\psi_{i}^{*}$ be a positive eigenfunction of $\lambda^{*}\left(d_{i}, \beta_{i}-\gamma_{i}\right)$ satisfying $\max _{x \in \bar{\Omega}} \psi_{i}^{*}(x)=1$. Recall that $1-\mathcal{R}_{0, i}$ and $\lambda^{*}\left(d_{i}, \beta_{i}-\gamma_{i}\right)$ have the same sign. We can now complete the proof of Theorems 2.5 and 2.6 .

Proof of Theorem 2.5 Thanks to Lemma 5.6, we know that there exists $S^{*}(\cdot)$ such that $\left\|S(t, \cdot)-S^{*}(\cdot)\right\|_{\infty}+\sum_{i=1}^{k}\left\|I_{i}(t, \cdot)\right\|_{\infty} \rightarrow 0$ as $t \rightarrow \infty$. It clearly follows from (13) that $\int_{\Omega} S^{*}=N$. Now, set $J^{+}:=\left\{x \in \Omega: S^{*}(x)=0\right\}$ and we show that $(i)-($ ii $)$ hold. First, it follows from the proof of Lemma 5.6 that $S^{*}(x)>S(0, x)>0$ for every $x \in \cap_{i=1}^{k}\left(\Omega \backslash H_{i}^{+}\right)=\Omega \backslash \cup_{i=1}^{k} H_{i}^{+}$. Hence, $J^{+} \subset \cup_{i} H_{i}^{+}$.

(i) Suppose that $\mathcal{R}_{0}>1$. Since $\mathcal{R}_{0}>1$, without loss of generality, we may suppose that $\mathcal{R}_{0,1}>1$ and we proceed to show that $\left|J^{+}\right|$is positive. Let $\left(\lambda^{*}\left(d_{1}, \beta_{1}-\gamma_{1}\right), \psi_{1}^{*}\right)$ be given above. Since $\mathcal{R}_{0,1}>1$, we know that $\lambda^{*}\left(d_{1}, \beta_{1}-\gamma_{1}\right)<0$. For any $\varepsilon>0$, define

$$
\Omega_{\varepsilon}:=\left\{x \in \bar{\Omega}: S^{*}(x)>2 \varepsilon\right\}
$$

It is easy to see that $J^{+}:=\cap_{\varepsilon>0}\left(\Omega \backslash \Omega_{\varepsilon}\right)$ and $\left|J^{+}\right|=\lim _{\varepsilon \rightarrow 0}\left|\bar{\Omega} \backslash \Omega_{\varepsilon}\right|$. Recalling that $\left(S(t, \cdot), I_{1}(t, \cdot), \ldots, I_{k}(t, \cdot)\right) \rightarrow\left(S^{*}(\cdot), 0, \ldots, 0\right)$ as $t \rightarrow \infty$ uniformly in $x \in \Omega$, 
then for every $\varepsilon>0$, there is $t_{\varepsilon} \gg 1$ such that

$$
S(t, x)>\varepsilon \text { and }\left\|\sum_{i=1}^{k} I_{i}(t, \cdot)\right\|_{\infty} \leq \varepsilon^{2} \quad \forall x \in \Omega_{\varepsilon}, t \geq t_{\varepsilon}
$$

Letting $C=\frac{\tilde{c}_{1}\left\|\psi^{*}\right\|_{\infty}}{|\Omega|^{2} \min _{x \in \bar{\Omega}} \psi^{*}(x)}$, where $\tilde{c}_{1}$ is the positive constant of (16), it holds for every $t \geq t_{\varepsilon}$ that

$$
\begin{aligned}
& \int_{\Omega} \frac{\beta_{1} \sum_{i=1}^{k} I_{i}(t)}{\sum_{i=1}^{k} I_{i}(t)+S(t)} I_{1}(t) \psi_{1}^{*} \\
& \quad=\int_{\Omega_{\varepsilon}} \frac{\beta_{1} \sum_{i=1}^{k} I_{i}(t)}{\sum_{i=1}^{k} I_{i}(t)+S(t)} I_{1}(t) \psi_{1}^{*}+\int_{\Omega \backslash \Omega_{\varepsilon}} \frac{\beta_{1} \sum_{i=1}^{k} I_{i}(t)}{\sum_{i=1}^{k} I_{i}(t)+S(t)} I_{1}(t) \psi_{1}^{*} \\
& \leq\left\|\beta_{1}\right\|_{\infty} \int_{\Omega_{\varepsilon}} \frac{\sum_{i=1}^{k} I_{i}(t)}{S(t)} I_{1}(t) \psi_{1}^{*}+\left\|\beta_{1}\right\|_{\infty}\left|\Omega \backslash \Omega_{\varepsilon}\right|\left\|I_{1}(t)\right\|_{\infty}\left\|\psi_{1}^{*}\right\|_{\infty} \\
& \quad \leq \varepsilon\left\|\beta_{1}\right\|_{\infty} \int_{\Omega_{\varepsilon}} I_{1}(t) \psi_{1}^{*}+C\left\|\beta_{1}\right\|_{\infty}\left|\Omega \backslash \Omega_{\varepsilon}\right| \int_{\Omega} I_{1}(t) \psi_{1}^{*} \\
& \quad \leq\left\|\beta_{1}\right\|_{\infty}\left(\varepsilon+C\left|\Omega \backslash \Omega_{\varepsilon}\right|\right) \int_{\Omega} I_{1}(t) \psi_{1}^{*} .
\end{aligned}
$$

Multiplying the second equation of (1) by $\psi_{1}^{*}$ and integrating by parts, we get

$$
\begin{aligned}
\frac{\mathrm{d}}{\mathrm{d} t} \int_{\Omega} I_{1}(t) \psi_{1}^{*}= & -d_{1} \int_{\Omega} \nabla \psi_{1}^{*} \nabla I_{1}(t) \\
& +\int_{\Omega}\left(\beta_{1}-\gamma_{1}-\frac{\beta_{1} \sum_{i=1}^{k} I_{i}(t)}{S(t)+\sum_{i=1}^{k} I_{i}(t)}\right) I_{1}(t) \psi_{1}^{*} \\
\geq & d_{1} \int_{\Omega} \nabla \psi_{1}^{*} \nabla I_{1}(t) \\
& +\int_{\Omega}\left(\beta_{1}-\gamma_{1}\right) I_{1}(t) \psi_{1}^{*}-\left\|\beta_{1}\right\|_{\infty}\left(\varepsilon+C\left|\Omega \backslash \Omega_{\varepsilon}\right|\right) \int_{\Omega} I_{1}(t) \psi_{1}^{*}
\end{aligned}
$$

for every $t \geq t_{\varepsilon}$. On the other hand, multiplying (20) by $I_{1}(t, x)$ and integrating by parts, we get

$-\lambda^{*}\left(d_{1}, \beta_{1}-\gamma_{1}\right) \int_{\Omega} I_{1}(t) \psi_{1}^{*}=-d_{1} \int_{\Omega} \nabla \psi_{1}^{*} \nabla I_{1}(t)+\int_{\Omega}\left(\beta_{1}-\gamma_{1}\right) I_{1}(t) \psi_{1}^{*} \forall t>0$.

By (74) and (75),

$$
\frac{\mathrm{d} t}{\mathrm{~d} t} \int_{\Omega} I_{1}(t) \psi_{1}^{*} \geq-\left(\lambda^{*}\left(d_{1}, \beta_{1}-\gamma_{1}\right)+\left\|\beta_{1}\right\|_{\infty}\left(\varepsilon+C\left|\Omega \backslash \Omega_{\varepsilon}\right|\right)\right) \int_{\Omega} I_{1}(t) \psi_{1}^{*}
$$


for every $t \geq t_{\varepsilon}$. Thus,

$$
\begin{aligned}
& \frac{1}{t} \ln \left(\frac{\left\|I_{1}\left(t+t_{\varepsilon}\right) \psi_{1}^{*}\right\|_{L^{1}(\Omega)}}{\left\|I_{1}\left(t_{\varepsilon}\right) \psi_{1}^{*}\right\|_{L^{1}(\Omega)}}\right) \\
& \quad \geq-\left(\lambda^{*}\left(d_{1}, \beta_{1}-\gamma_{1}\right)+\left\|\beta_{1}\right\|_{\infty}\left(\varepsilon+C\left|\Omega \backslash \Omega_{\varepsilon}\right|\right)\right) \quad \forall t>0, \varepsilon>0 .
\end{aligned}
$$

Since $\sup _{t \geq 0}\left\|I_{1}(t)\right\|_{\infty}<\infty$, we derive from the last inequality that

$$
\varepsilon+C\left|\Omega \backslash \Omega_{\varepsilon}\right| \geq \frac{-\lambda^{*}\left(d_{1}, \beta_{1}-\gamma_{1}\right)}{\left\|\beta_{1}\right\|_{\infty}} \quad \varepsilon>0
$$

Letting $\varepsilon \rightarrow 0$ yields that $\left|J^{+}\right| \geq \frac{-\lambda^{*}\left(d_{1}, \beta_{1}-\gamma_{1}\right)}{\left\|\beta_{1}\right\|_{\infty} C}>0$. Hence, $J^{+}$has a positive measure. It is easy to see that if $x \in \Omega \backslash \cup_{i=1}^{k} H_{i}^{+}$the function $t \mapsto S(t, x)$ is increasing, and hence $x \in \Omega \backslash J^{+}$. This shows that $J^{+}$is contained in $\cup_{i=1}^{k}\left(\Omega \cap H_{i}^{+}\right)$. Next, observe that $J^{+}$is a closed subset of $\Omega$ and that $\cup_{i=1}^{k}\left(\Omega \cap H_{i}^{+}\right)$is an open subset of $\Omega$. Hence, if $J^{+}=\cup_{i=1}^{k}\left(\Omega \cap H_{i}^{+}\right)$, we would then have that $\Omega=J^{+}=\cup_{i=1}^{k}\left(\Omega \cap H_{i}^{+}\right)$, since $\Omega$ is a connected set. And hence $S^{*} \equiv 0$, which is impossible since $\int_{\Omega} S^{*}=N>0$. Whence, we must have that $J^{+} \varsubsetneqq \cup_{i=1}^{k}\left(\Omega \cap H_{i}^{+}\right)$, that is $\cup_{i=1}^{k}\left(\Omega \cap H_{i}^{+}\right) \backslash J^{+}$is a nonempty open subset of $\Omega$. So, we can conclude that the set $\cup_{i=1}^{k}\left(\Omega \cap H_{i}^{+}\right) \backslash J^{+}$ also has a positive measure being a nonempty open set.

(ii) Assume that $\mathcal{R}_{0}<1$. Let $\left(\lambda^{*}\left(d_{i}, \beta_{i}-\gamma_{i}\right), \psi_{i}^{*}\right)$ be given above for each $i=1, \ldots, k$. We know that $\lambda^{*}\left(d_{i}, \beta_{i}-\gamma_{i}\right)>0$ for each $i=1, \ldots, k$. It is easy to see that

$$
\partial_{t} I_{i} \leq d_{i} \Delta I_{i}+\left(\beta_{i}-\gamma_{i}\right) I_{i} \quad x \in \Omega, t>0 .
$$

So, by the comparison principle for parabolic equations,

$$
I_{i}(t, x) \leq\left\|I_{i}(0, \cdot)\right\| e^{-\lambda^{*}\left(d_{i}, \beta_{i}-\gamma_{i}\right) t} \frac{\psi_{i}^{*}(x)}{\min _{z \in \bar{\Omega}} \psi_{i}^{*}(z)} \quad \forall x \in \Omega, t>0, i=1 \cdots, k
$$

Taking $M=\sum_{i=1}^{k} \frac{1}{\min _{z \in \bar{\Omega}} \psi_{i}^{*}(z)}$, we obtain that

$$
\begin{aligned}
\int_{0}^{\infty}\left\|S_{t}(s, \cdot)\right\|_{\infty} \mathrm{d} s & \leq M \sum_{i=1}^{k}\left\|\beta_{i}+\gamma_{i}\right\|_{\infty}\left\|\sum_{i=1}^{k} I_{i}(0, \cdot)\right\|_{\infty} \int_{0}^{\infty} e^{-\min _{1 \leq i \leq k} \lambda^{*}\left(d_{i}, \beta_{i}-\gamma_{i}\right) s} \mathrm{~d} s \\
& =\frac{M}{\min _{1 \leq i \leq k} \lambda^{*}\left(d_{i}, \beta_{i}-\gamma_{i}\right)} \sum_{i=1}^{k}\left\|\beta_{i}+\gamma_{i}\right\|_{\infty}\left\|\sum_{i=1}^{k} I_{i}(0, \cdot)\right\|_{\infty} .
\end{aligned}
$$


Hence, $\forall x \in \Omega$,

$$
\begin{aligned}
S^{*}(x) & \geq S(0, x)-\frac{M}{\min _{1 \leq i \leq k} \lambda^{*}\left(d_{i}, \beta_{i}-\gamma_{i}\right)} \sum_{i=1}^{k}\left\|\beta_{i}+\gamma_{i}\right\|_{\infty}\left\|\sum_{i=1}^{k} I_{i}(0, \cdot)\right\|_{\infty} \\
& \geq \min _{z \in \bar{\Omega}} S(0, z)-\frac{M}{\min _{1 \leq i \leq k} \lambda^{*}\left(d_{i}, \beta_{i}-\gamma_{i}\right)} \sum_{i=1}^{k}\left\|\beta_{i}+\gamma_{i}\right\|_{\infty}\left\|\sum_{i=1}^{k} I_{i}(0, \cdot)\right\|_{\infty} .
\end{aligned}
$$

We may take $m_{0}=\left[\frac{M}{\min _{1 \leq i \leq k} \lambda^{*}\left(d_{i}, \beta_{i}-\gamma_{i}\right)} \sum_{i=1}^{k}\left\|\beta_{i}+\gamma_{i}\right\|_{\infty}\right]^{-1}$ and obtain from the above inequality that $J^{+}=\emptyset$ whenever $\left\|\sum_{i=1}^{k} I_{i}(0, \cdot)\right\|_{\infty}<m_{0} S(0, \cdot)$.

Proof of Theorem 2.6 Suppose that $k=1$. (i) It is easy to check given any $S^{*} \in C(\bar{\Omega})$ satisfying (8), $\left(S^{*}(x), 0\right)$ is always a solution of (1). Next, let $\left(S(x), I_{1}(x)\right)$ be a steady-state solution of (1). The maximum principle implies that $I_{1}(x)>0$ for every $x \in \Omega$. Hence, it follows from the equation satisfied by $S(x)$ that either $I_{1}(x) \equiv 0$ or $I_{1}(x) \gamma_{1}(x)=\left(\beta_{1}(x)-\gamma_{1}(x)\right) S(x)$ for every $x \in \Omega$. It is clear that $I_{1}(x) \equiv 0$ implies that $S(x)$ must satisfy (8). In the second case, that is, $I_{1}(x) \gamma_{1}(x)=\left(\beta_{1}(x)-\gamma_{1}(x)\right) S(x)$ for every $x \in \Omega$, since $\mathfrak{R}_{0}<1$ and hence $H^{-}$is nonempty, then there is an element $x_{0} \in \Omega$ such that $I_{1}\left(x_{0}\right)=0$. As a result, we must have that $I_{1}(x) \equiv 0$, so we are back to the first case. This completes the proof of $(i)$.

(ii) Suppose that $\mathfrak{R}_{0}>1$, and hence $\beta_{1}(x)>\gamma_{1}(x)$ for every $x \in \bar{\Omega}$. It is easy to check that $\left(S_{e}(x), I_{e}(x)\right)$ defined by (9) is a steady state solution of (1). Next, let $\left(S(x), I_{1}(x)\right)$ be a steady-state solution of (1) for which $I_{1}(x)>0$ for every $x \in \Omega$. Hence, from the equation satisfied by $S(x)$, we must have

$$
S(x)=\frac{\gamma_{1}(x)}{\beta_{1}(x)-\gamma_{1}(x)} I_{1}(x)=\frac{I_{1}(x)}{\mathfrak{R}_{1}(x)-1} \quad \forall x \in \Omega .
$$

Thus, $I_{1}(x)$ must satisfy

$$
\begin{cases}0=d_{1} \Delta I_{1} & x \in \Omega \\ \partial_{\vec{n}} I=0 & x \in \partial \Omega\end{cases}
$$

which implies that $I_{1}(x)=$ constant. This together with the equation $N=\int_{\Omega}\left(I_{1}(x)+\right.$ $S(x)$ ) yields that

$$
N=\int_{\Omega}\left(1+\frac{\gamma_{1}(x)}{\beta_{1}(x)-\gamma_{1}(x)}\right) I_{1} \mathrm{~d} x=I_{1} \int_{\Omega} \frac{\mathfrak{R}_{1}(x)}{\Re_{1}(x)-1} \mathrm{~d} x .
$$

Solving for $I_{1}$ in the last equation, we obtain that $I_{1}=I_{e}$.

Suppose that $k=1$ and that $\Re_{0}>1$ and we present the proof of Theorem 2.8. To this end, we first study the large time behavior of solution of the following related 
system:

$$
\begin{cases}\tilde{S}_{t}=(\tilde{I}-\tilde{S}) \frac{\rho_{1}(x) \tilde{I}}{\rho(x) \tilde{S}+\tilde{I}} & x \in \Omega \\ \tilde{I}_{t}=d_{1} \Delta \tilde{I}+(\tilde{S}-\tilde{I}) \frac{\rho_{2}(x) \tilde{I}}{\rho(x) \tilde{S}+\tilde{I}} & x \in \Omega \\ \partial_{\vec{n}} \tilde{I}=0 & x \in \Omega\end{cases}
$$

where $\rho, \rho_{1}, \rho_{2} \in C^{\alpha}(\bar{\Omega}), \min _{x \in \Omega} \rho(x)>0$ and $\min _{x \in \Omega} \rho_{i}(x)>0$ for each $i=1,2$. Given positive initial functions $\left(\tilde{S}_{0}(x), \tilde{I}_{0}(x)\right) \in[C(\bar{\Omega})]^{2}$, we denote by $\left(\tilde{S}\left(t, x ; \tilde{S}_{0}, \tilde{I}_{0}\right), \tilde{I}\left(t, x ; \tilde{S}_{0}, \tilde{I}_{0}\right)\right)$ the unique classical solution of $(76)$. By the comparison principle, it holds that $\tilde{S}(t, x)>0$ and $\tilde{I}(t, x)>0$ for every $t>0$ and $x \in \Omega$. Moreover, $\sup _{t \geq 0}\|\tilde{S}(t, \cdot)+\tilde{I}(t, \cdot)\|_{\infty}<\infty$. It is easy to see that (76) has infinitely many steady state solutions which are all constant functions of the form $(\tilde{S}(t, x), \tilde{I}(t, x)) \equiv(c, c)$ where $c$ is some positive constant. Our aim is first to study the large time behavior of solutions to (76) and next deduce the asymptotic behavior of solutions to (1) when $H_{1}^{+}=\bar{\Omega}$. The following result holds for (76).

Lemma 5.7 Let $\left(\tilde{S}\left(t, x ; \tilde{S}_{0}, \tilde{I}_{0}\right), \tilde{I}\left(t, x ; \tilde{S}_{0}, \tilde{I}_{0}\right)\right)$ be a classical solution of (76) with $\left(\tilde{S}_{0}, \tilde{I}_{0}\right) \in[C(\bar{\Omega})]^{2}$ and $\tilde{I}_{0}(x)>0$ and $\tilde{S}_{0}(x)>0$ for every $x \in \bar{\Omega}$, and define

$$
\underline{c}(t)=\min \left\{\min _{x \in \bar{\Omega}} \tilde{S}\left(t, x ; \tilde{S}_{0}, \tilde{I}_{0}\right), \min _{x \in \bar{\Omega}} \tilde{I}\left(t, x ; \tilde{S}_{0}, \tilde{I}_{0}\right)\right\} \quad \forall t \geq 0
$$

and

$$
\bar{c}(t)=\max \left\{\max _{x \in \bar{\Omega}} \tilde{S}\left(t, x ; \tilde{S}_{0}, \tilde{I}_{0}\right), \max _{x \in \bar{\Omega}} \tilde{I}\left(t, x ; \tilde{S}_{0}, \tilde{I}_{0}\right)\right\} \quad \forall t \geq 0
$$

Then, the following conclusions hold:

(i) The map $t \mapsto \underline{c}(t)$ is monotone increasing and the map $t \mapsto \bar{c}(t)$ is monotone decreasing.

(ii) If we denote by $\underline{c}=\lim _{t \rightarrow \infty} \underline{c}(t)$ and $\bar{c}=\lim _{t \rightarrow \infty} \bar{c}(t)$ then

$$
\liminf _{t \rightarrow \infty} \min _{x \in \bar{\Omega}} \tilde{S}\left(t, x ; \tilde{S}_{0}, \tilde{I}_{0}\right)=\liminf _{t \rightarrow \infty} \min _{x \in \bar{\Omega}} \tilde{I}\left(t, x ; \tilde{S}_{0}, \tilde{I}_{0}\right)=\underline{c}
$$

and

$$
\limsup _{t \rightarrow \infty} \max _{x \in \bar{\Omega}} \tilde{S}\left(t, x ; \tilde{S}_{0}, \tilde{I}_{0}\right)=\limsup _{t \rightarrow \infty} \max _{x \in \bar{\Omega}} \tilde{I}\left(t, x ; \tilde{S}_{0}, \tilde{I}_{0}\right)=\bar{c}
$$

Proof $(i)$ Define the functions

$$
F_{i}(t, x)=\frac{\rho_{i}(x) \tilde{I}\left(t, x ; \tilde{S}_{0}, \tilde{I}_{0}\right)}{\tilde{I}\left(t, x ; \tilde{S}_{0}, \tilde{I}_{0}\right)+\rho(x) \tilde{S}\left(t, x ; \tilde{S}_{0}, \tilde{I}_{0}\right)}
$$


for every $i=1,2$, we have that $F_{i}(t, x)>0$ for every $x \in \bar{\Omega}, t \geq 0$ and $i=1,2$. Moreover, $\left(\tilde{S}\left(t, x ; \tilde{S}_{0}, \tilde{I}_{0}\right), \tilde{I}\left(t, x ; \tilde{S}_{0}, \tilde{I}_{0}\right)\right)$ is a classical solution of the cooperative system

$$
\begin{cases}\partial_{t} S=(I-S) F_{1}(t, x) & x \in \Omega \\ \partial_{t} I=d_{1} \Delta I+(S-I) F_{2}(t, x) & x \in \Omega \\ \partial_{\vec{n}} I=0 & x \in \partial \Omega .\end{cases}
$$

Next, for every $t_{0} \geq 0$, both the constant functions $\left(\underline{c}\left(t_{0}\right), \underline{c}\left(t_{0}\right)\right)$ and $\left(\bar{c}\left(t_{0}\right), \bar{c}\left(t_{0}\right)\right)$ are classical solutions of (77) on $\Omega \times\left(t_{0}, \infty\right)$ satisfying

$$
\left(\underline{c}\left(t_{0}\right), \underline{c}\left(t_{0}\right)\right) \leq\left(\tilde{S}\left(t_{0}, x ; \tilde{S}_{0}, \tilde{I}_{0}\right), \tilde{I}\left(t_{0}, x ; \tilde{S}_{0}, \tilde{I}_{0}\right)\right) \leq\left(\bar{c}\left(t_{0}\right), \bar{c}\left(t_{0}\right)\right) \quad \forall x \in \Omega,
$$

where the inequalities hold component-wise. Hence, by the comparison principle for cooperative systems, it holds that

$$
\left(\underline{c}\left(t_{0}\right), \underline{c}\left(t_{0}\right)\right) \leq\left(\tilde{S}\left(t, x ; \tilde{S}_{0}, \tilde{I}_{0}\right), \tilde{I}\left(t, x ; \tilde{S}_{0}, \tilde{I}_{0}\right)\right) \leq\left(\bar{c}\left(t_{0}\right), \bar{c}\left(t_{0}\right)\right) \quad \forall x \in \Omega, t \geq t_{0} .
$$

Since $t_{0}$ is arbitrary given, we conclude that $(i)$ holds.

(ii) We note from $(i)$ that $\inf _{x, \in \Omega, t \geq 0} F_{i}(t, x)>0$ for every $i=1,2$. So, we can employ comparison principle to deduce $(i i)$ from $(i)$.

Lemma 5.8 Let $\left(\tilde{S}\left(t, x ; \tilde{S}_{0}, \tilde{I}_{0}\right), \tilde{I}\left(t, x ; \tilde{S}_{0}, \tilde{I}_{0}\right)\right)$ be a classical solution of (76) with $\left(\tilde{S}_{0}, \tilde{I}_{0}\right) \in[C(\bar{\Omega})]^{2}$ and $\tilde{I}_{0}(x)>0$ and $\tilde{S}_{0}(x)>0$ for every $x \in \bar{\Omega}$. Then

$$
\begin{aligned}
& \frac{1}{2} \frac{d}{d t}\left\{\int_{\Omega} \frac{\rho_{2}(x)}{\rho_{1}(x)} \tilde{S}^{2}(t, x) d x+\int_{\Omega} \tilde{I}^{2}(t, x) d x\right\} \\
& \quad=-\left\{\int_{\Omega} \frac{(\tilde{S}(t)-\tilde{I}(t))^{2} \tilde{I}(t, x) \rho_{2}(x)}{\rho(x) \tilde{S}(t, x)+\tilde{I}(t, x)} d x+d_{1} \int_{\Omega}|\nabla \tilde{I}(t, x)|^{2} d x\right\} \forall t>0 .
\end{aligned}
$$

Proof Using (76) and integration by parts, we get

$$
\frac{1}{2} \frac{\mathrm{d}}{\mathrm{d} t} \int_{\Omega} \frac{\rho_{2}(x)}{\rho_{1}(x)} \tilde{S}^{2} \mathrm{~d} x=\int_{\Omega} \frac{\rho_{2}(x)}{\rho_{1}(x)} \tilde{S} \tilde{S}_{t}=\int_{\Omega} \frac{\rho_{2}(x)(\tilde{I}-\tilde{S}) \tilde{I} \tilde{S}}{\tilde{I}+\rho(x) \tilde{S}}
$$


and

$$
\begin{aligned}
\frac{1}{2} \frac{\mathrm{d}}{\mathrm{d} t} \int_{\Omega} \tilde{I}^{2} \mathrm{~d} x & =\int_{\Omega} \tilde{I}_{t} \tilde{I} \\
& =\int_{\Omega} \tilde{I}\left(d_{I} \Delta \tilde{I}+(\tilde{S}-\tilde{I}) \frac{\rho_{2}(x) \tilde{I}}{\rho(x) \tilde{S}+\tilde{I}}\right) \\
& =-d_{1} \int_{\Omega}|\nabla \tilde{I}|^{2}-\int_{\Omega} \frac{\rho_{2}(x)(\tilde{I}-\tilde{S}) \tilde{I}^{2}}{\rho(x) \tilde{S}+\tilde{I}} .
\end{aligned}
$$

Adding up both (79) and (80) yield (78).

Thanks to Lemma 5.8, the large time behavior of solutions to (76) can be studied. Theorem 5.9 Let $\left(\tilde{S}\left(t, x ; \tilde{S}_{0}, \tilde{I}_{0}\right), \tilde{I}\left(t, x ; \tilde{S}_{0}, \tilde{I}_{0}\right)\right)$ be a classical solution of $(76)$ with $\left(\tilde{S}_{0}, \tilde{I}_{0}\right) \in[C(\bar{\Omega})]^{2}$ and $\tilde{I}_{0}(x)>0$ and $\tilde{S}_{0}(x)>0$ for every $x \in \Omega$. Then

$$
\lim _{t \rightarrow \infty}\left\|\tilde{S}\left(t, \cdot ; \tilde{S}_{0}, \tilde{I}_{0}\right)-c\right\|_{L^{2}(\Omega)}=\lim _{t \rightarrow \infty}\left\|\tilde{I}\left(t, \cdot ; \tilde{S}_{0}, \tilde{I}_{0}\right)-c\right\|_{\infty}=0
$$

where the constant $c$ is uniquely determined by

$$
c=\frac{1}{\int_{\Omega}\left(\frac{\rho_{2}(x)}{\rho_{1}(x)}+1\right) d x} \int_{\Omega}\left[\frac{\rho_{2}(x)}{\rho_{1}(x)} \tilde{S}_{0}(x)+\tilde{I}_{0}(x)\right] d x
$$

Proof The proof is divided into two steps.

Step 1 In this step, we show that

$$
\lim _{t \rightarrow \infty}\left\{\int_{\Omega}(\tilde{S}(t, x)-\tilde{I}(t, x))^{2} \mathrm{~d} x+\int_{\Omega}|\nabla \tilde{I}(t, x)|^{2} \mathrm{~d} x\right\}=0
$$

We recall from Lemma 5.7 that there are positive constants $0<c_{*}<c^{*}$ such that $c_{*} \leq \tilde{I}(t, x), \tilde{S}(t, x) \leq c^{*}$ for every $x \in \Omega$ and $t \geq 0$. Thus,

$$
\int_{\Omega} \frac{(\tilde{S}(t, x)-\tilde{I}(t, x))^{2} \tilde{I}(t, x) \rho_{2}(x)}{\rho(x) \tilde{S}(t, x)+\tilde{I}(t, x)} \mathrm{d} x \geq m \int_{\Omega}(\tilde{S}(t)-\tilde{I}(t))^{2} \mathrm{~d} x \quad \forall t \geq 0,(84)
$$

where $m:=\frac{c_{*} \min _{x \in \bar{\Omega}} \rho_{2}(x)}{c^{*}\left(1+\min _{x \in \bar{\Omega}} \rho(x)\right)}>0$. Observe from (78) that

$$
\int_{0}^{\infty}\left\{\int_{\Omega} \frac{(\tilde{S}(t, x)-\tilde{I}(t, x))^{2} \tilde{I}(t, x) \rho_{2}(x)}{\rho(x) \tilde{S}(t, x)+\tilde{I}(t, x)} \mathrm{d} x+d_{1} \int_{\Omega}|\nabla \tilde{I}(t, x)|^{2} \mathrm{~d} x\right\} \mathrm{d} t<\infty .
$$


Whence, by (84), we also get that

$$
\int_{0}^{\infty}\left\{\int_{\Omega}(\tilde{S}(t, x)-\tilde{I}(t, x))^{2} \mathrm{~d} x+\int_{\Omega}|\nabla \tilde{I}(t, x)|^{2} \mathrm{~d} x\right\} \mathrm{d} t<\infty .
$$

Now, note that $\sup _{t \geq 0, x \in \Omega} \frac{|\tilde{S}(t, x)-\tilde{I}(t, x)| \rho_{2}(x)}{\rho(x) \tilde{S}(t, x)+\tilde{I}(t, x)} \leq \frac{2 c^{*}\left\|\rho_{2}\right\|_{\infty}}{\left(1+\min _{x \in \bar{\Omega}} \rho(x)\right) c_{*}}<\infty$. Thanks to regularity theory for parabolic equations, we have that the map

$$
[1, \infty) \ni t \mapsto\|\tilde{I}\|_{C^{1}(\bar{\Omega})}
$$

is uniformly Hölder continuous. This in turn implies that the map

$$
[1, \infty) \ni t \mapsto\|\nabla \tilde{I}\|_{L^{2}(\Omega)}
$$

is uniformly Hölder continuous. Thus, since

$$
\int_{0}^{\infty} \int_{\Omega}|\nabla \tilde{I}(t, x)|^{2} \mathrm{~d} x \mathrm{~d} t<\infty, \quad(\operatorname{see}(85))
$$

we deduce that

$$
\lim _{t \rightarrow \infty} \int_{\Omega}|\nabla \tilde{I}(t, x)|^{2} \mathrm{~d} x=0
$$

Next, taking $G(t)=\|I(\tilde{t}, \cdot)-\tilde{S}(t, \cdot)\|_{L^{2}(\Omega)}^{2}$ for every $t \geq 0$ and $M=$ $\sup _{t \geq 0, x \in \Omega}\left|\tilde{S}_{t}(t, x)\right| \leq \frac{2 c^{*}\left\|\rho_{2}\right\|_{\infty}}{\left(1+\min _{x \in \bar{\Omega}} \rho(x)\right) c_{*}}<\infty$, we get

$$
\begin{aligned}
|G(t+h)-G(t)| & \leq 4 c^{*} \int_{\Omega}(|\tilde{I}(t+h, x)-\tilde{I}(t, x)|+|\tilde{S}(t+h, x)-\tilde{S}(t, x)|) \mathrm{d} x \\
& \leq 4 c^{*} \int_{\Omega}(|\tilde{I}(t+h, x)-\tilde{I}(t, x)|+M|h|) \mathrm{d} x \quad \forall t \geq 1, h>0 .
\end{aligned}
$$

Similarly, using the fact that the map $[1, \infty) \ni t \mapsto\|\tilde{I}\|_{C^{1}(\bar{\Omega})}$ is uniformly Hölder continuous, so there is $v \in(0,1)$, there is $M_{v}>0$ such that

$$
\|\tilde{I}(t+h, \cdot)-\tilde{I}(t, \cdot)\|_{\infty} \leq M_{\nu}|h|^{\nu} .
$$

Whence, inequality (87) can be improved to

$$
|G(t+h)-G(t)| \leq 4 c^{*}|\Omega|\left(M_{v}|h|^{\nu}+M|h|\right), \quad \forall t \geq 1, h \geq 0 .
$$


Therefore, since $\int_{0}^{\infty} G(t) \mathrm{d} t<\infty$ by (85) and, $G(t) \geq 0$ for every $t \geq 0$, we conclude that

$$
\lim _{t \rightarrow \infty} G(t)=0
$$

This together with (86) yields (83).

Step 2 In this step, we show that

$$
\lim _{t \rightarrow \infty}\|\tilde{I}(t, \cdot)-c\|_{\infty}=0
$$

where $c$ is given by (82). We proceed by contradiction to show (88). So, suppose that there is some sequence $\left\{t_{n}\right\}_{n \geq 1}$ with $t_{n} \rightarrow \infty$ such that

$$
\inf _{n \geq 1}\left\|\tilde{I}\left(t_{n}, \cdot\right)-c\right\|_{\infty}>0
$$

Since the function $\tilde{I}(t, \cdot)$ is uniformly Hölder continuous on $[1, \infty)$ with respect to $\|\cdot\|_{C^{1}(\bar{\Omega})}$ with $\sup _{t \geq 1}\|\tilde{I}(t, \cdot)\|_{C^{1}(\bar{\Omega})}<\infty$, then by the Arzella-Ascoli's theorem, there is a subsequence $\left\{t_{n, 1}\right\}$ of $\left\{t_{n}\right\}_{n \geq 1}$ such that $\tilde{I}\left(t_{n, 1}, \cdot\right) \rightarrow I^{*}(\cdot)$ in $C^{1}(\bar{\Omega})$ as $n \rightarrow \infty$. In particular, it holds that $\tilde{I}\left(t_{n, 1}, \cdot\right) \rightarrow I^{*}(\cdot)$ as $n \rightarrow \infty$ in $W^{1,2}(\Omega)$. Thus, by Step 1, we conclude that $\left\|\left|\nabla I^{*}\right|\right\|_{L^{2}(\Omega)}=0$ and $\left\|\tilde{S}\left(t_{n, 1}, \cdot\right)-I^{*}\right\|_{L^{2}(\Omega)} \rightarrow 0$ as $n \rightarrow \infty$. Hence $I^{*}=$ constant and $\left(\tilde{S}\left(t_{n, 1}, \cdot\right), \tilde{I}\left(t_{n, 1}, \cdot\right)\right) \rightarrow\left(I^{*}, I^{*}\right)$ as $n \rightarrow \infty$ in $L^{2}(\Omega)$. This implies that

$$
\lim _{n \rightarrow \infty} \int_{\Omega}\left(\frac{\rho_{2}(x)}{\rho_{1}(x)} \tilde{S}\left(t_{n, 1}, x\right)+\tilde{I}\left(t_{n, 1}, x\right)\right) \mathrm{d} x=I^{*} \int_{\Omega}\left(\frac{\rho_{2}(x)}{\rho_{1}(x)}+1\right) \mathrm{d} x
$$

Next, it is easy to see that

$$
\frac{\mathrm{d}}{\mathrm{d} t} \int_{\Omega}\left[\frac{\rho_{2}(x)}{\rho_{1}(x)} \tilde{S}(t, x)+\tilde{I}(t, x)\right] \mathrm{d} x=0 \quad \forall t>0 .
$$

Thus,

$$
\int_{\Omega}\left[\frac{\rho_{2}(x)}{\rho_{1}(x)} \tilde{S}_{0}(x)+\tilde{I}_{0}(x)\right] \mathrm{d} x=\int_{\Omega}\left[\frac{\rho_{2}(x)}{\rho_{1}(x)} \tilde{S}(t, x)+\tilde{I}(t, x)\right] \mathrm{d} x \quad \forall t \geq 0 .
$$

Combining (90) and (91), we obtain that the constant $I^{*}$ is given by (82). Thus, we conclude that $\left\|\tilde{I}\left(t_{n, 1}, \cdot\right)-c\right\|_{\infty}=0$, which contradicts (89). Thus, the statement (88) holds. Now, we can easily derive both (81) and (82) from Steps 1 and 2.

Now, using Theorem 5.9, we are able to complete the proof of Theorem 2.8.

Proof of Theorem 2.8 Define

$$
\tilde{S}(t, x)=\frac{S(t, x)}{S_{e}(x)} \text { and } \tilde{I}(t, x)=\frac{I(t, x)}{I_{e}} \quad \forall x \in \Omega, t \geq 0 .
$$


Taking $\rho(x)=\frac{S_{e}(x)}{I_{e}}, \rho_{1}(x)=\frac{\gamma_{1}(x) I_{e}}{S_{e}(x)}$ and $\rho_{2}(x)=\gamma_{1}(x)$ and noticing that $\beta_{1}(x)-$ $\gamma_{1}(x)=\frac{I_{e} \gamma_{1}(x)}{S_{e}(x)}$ for every $x \in \Omega$, one easily checks that $(\tilde{S}(t, x), \tilde{I}(t, x))$ solves (76). Hence, we may apply Theorem 5.9 to conclude that there is some constant $c$ such that

$$
\lim _{t \rightarrow \infty}\left[\|\tilde{S}(t, \cdot)-c\|_{L^{2}(\Omega)}+\|\tilde{I}(t, \cdot)-c\|_{\infty}\right]=0 .
$$

This is equivalent to saying that

$$
\lim _{t \rightarrow \infty}\left[\left\|S(t, \cdot)-c S_{e}(\cdot)\right\|_{L^{2}(\Omega)}+\left\|I(t, \cdot)-c I_{e}\right\|_{\infty}\right]=0
$$

where the constant $c$ in (82) is

$$
\begin{aligned}
c & =\frac{\int_{\Omega}\left[\frac{\rho_{2}}{\rho_{1}} \tilde{S}(0, \cdot)+\tilde{I}(0, \cdot)\right]}{\int_{\Omega}\left(\frac{\rho_{2}}{\rho_{1}}+1\right)} \\
& =\frac{\int_{\Omega}\left[\frac{\gamma_{1} S_{e}}{\gamma_{1} I_{e}} \frac{S(0, x)}{S_{e}}+\frac{I(0, x)}{I_{e}(x)}\right] \mathrm{d} x}{\int_{\Omega}\left(\frac{\gamma_{1} S_{e}}{\gamma_{1} I_{e}}+1\right)}=\frac{\int_{\Omega}(S(0, \cdot)+I(0, \cdot))}{\int_{\Omega}\left(S_{e}+I_{e}\right)}=\frac{N}{N}=1 .
\end{aligned}
$$

Next, we show that $\tilde{S}(t, x) \rightarrow 1$ as $t \rightarrow \infty$ uniformly in $x \in \Omega$. First, observe from Lemma 5.7(ii) and the fact that $\|\tilde{I}(t, \cdot)-1\|_{\infty} \rightarrow 0$ as $t \rightarrow \infty$, we obtain that

$$
\liminf _{t \rightarrow \infty} \min _{x \in \bar{\Omega}} \tilde{S}(t, x)=\limsup _{t \rightarrow \infty} \max _{x \in \bar{\Omega}} \tilde{S}(t, x)=1,
$$

that is $\|\tilde{S}(t, \cdot)-1\|_{\infty} \rightarrow 0$ as $t \rightarrow \infty$, which completes the proof.

\section{References}

Ackleh AS, Allen LJS (2003) Competitive exclusion and coexistence for pathogens in an epidemic model with variable population size. J Math Biol 47:153-168

Ackleh AS, Allen LJS (2005) Competitive exclusion in SIS and SIR epidemic models with total cross immunity and density-dependent host mortality. Discrete Contin Dyn Syst Ser B 5(2):175-188

Ackleh AS, Deng K, Wu Y (2016) Competitive exclusion and coexistence in a two-strain pathogen model with diffusion. Math Biosci Eng 13(1):1-18

Allen LSJ, Bolker BM, Lou Y, Nevai AL (2008) Asymptotic profiles of the steady states for an SIS epidemic reaction-diffusion model. Discrete Contin Dyn Syst 21(1):1-20

Bremermann HJ, Thieme HR (1989) A competitive exclusion principle for pathogen virulence. J Math Biol 27: $179-190$

Cantrell RS, Cosner C (2003) Spatial ecology via reaction-diffusion equations. Series in mathematical and computational biology. Wiley, Chichester

Cui R, Lam K-Y, Lou Y (2017) Dynamics and asymptotic profiles of steady states of an epidemic model in advective environments. J Differ Equ 263(4):2343-2373

Cui R, Lou Y (2016) A spatial SIS model in advective heterogeneous environments. J Differ Equ 261(4):3305-3343

Deng K (2019) Asymptotic behavior of an SIR reaction-diffusion model with a linear source. Discrete Contin Dyn Syst Ser B 24:5945-5957 
Deng K, Wu Y (2016) Dynamics of a susceptible-infected-susceptible epidemic reaction-diffusion model. Proc R. Soc Edinb Sect A 146:929-946

Gao D (2019) Travel frequency and infectious disease. SIAM J Appl Math 79:1581-1606

Ge J, Kim KI, Lin Z, Zhu H (2015) An SIS reaction-diffusion-advection model in a low-risk and high-risk domain. J Differ Equ 259(10):5486-5509

Henry D (1981) Geometric theory of semilinear parabolic equations. Springer, Berlin

Húska J (2006) Harnack inequality and exponential separation for oblique derivative problems on Lipschitz domains. J Differ Equ 226:541-557

Hess P (1991) Periodic-parabolic boundary value problems and positivity. Pitman Research Notes in Mathematics Series, Wiley

Levin SA, Pimentel D (1981) Selection of intermediate rates of increase in parasite-hosts systems. Am Nat 117:308-29

Li H, Peng R, Wang F-B (2017) Varying total population enhances disease persistence: qualitative analysis on a diffusive SIS epidemic model. J Differ Equ 262:885-913

Li H, Peng R, Xiang T (2020) Dynamics and asymptotic profiles of endemic equilibrium for two frequencydependent SIS epidemic models with cross-diffusion. Eur J Appl Math 31:25-56

Liu S, Lou Y (2021) Classifying the level sets of principal eigenvalue for time-periodic parabolic operators and applications (under review)

Mena-Lorca J, Velasco-Hernandez JX (1995) Superinfection, virulence and density dependent mortality in an epidemic model, Technical Report BU 1299-M. Cornell University, Biometrics Unit

Peng R (2009) Asymptotic profiles of the positive steady state for an SIS epidemic reaction-diffusion model I. J Differ Equ 247:1096-1119

Peng R, Liu S (2009) Global stability of the steady states of an SIS epidemic reaction-diffusion model. Nonlinear Anal 71:239-247

Peng R, Yi F (2013) Asymptotic profile of the positive steady state for an SIS epidemic reaction-diffusion model: effects of epidemic risk and population movement. Phys D 259:8-25

Peng R, Zhao XQ (2012) A reaction-diffusion SIS epidemic model in a time-periodic environment. Nonlinearity 25:1451-1471

Quittner P, Souplet P (2007) Superliner parabolic problems: blow-up, global existence and steady states. Birkhauser, Basel

Song P, Lou Y, Xiao Y (2018) A spatial SEIRS reaction-diffusion model in heterogeneous environment. J Differ Equ 267:5084-5114

Tuncer N, Martcheva M (2012) Analytical and numerical approaches to coexistence of strains in a two-strain SIS model with diffusion. J Biol Dyn 6:406-439

Wu Y, Tuncer N, Martcheva M (2017) Coexistence and competitive-exclusion in SIS model with standard incidence and diffusion. Discrete Contin Dyn Syst Ser B 22(7):1167-1187

Wu Y, Zou X (2016) Asymptotic profiles of steady states for a diffusive SIS epidemic model with mass action infection mechanism. J Differ Equ 261:4424-4447

Publisher's Note Springer Nature remains neutral with regard to jurisdictional claims in published maps and institutional affiliations. 